\title{
Total Synthesis and Initial Biological Evaluation of New B-Ring Modified Bryostatin Analogs
}

\author{
Paul A. Wender, * Joshua C. Horan, Vishal A. Verma \\ Department of Chemistry and Department of Molecular Pharmacology, \\ Stanford University, Stanford, CA 94305-5080
}

Supporting Information

General Methods. All glassware used was oven-dried $\left(>130{ }^{\circ} \mathrm{C}\right)$ and cooled under $\mathrm{N}_{2}$. Stirring was accomplished via magnetic, Teflon ${ }^{\circledR}$-coated stir bars that were oven-dried and cooled under $\mathrm{N}_{2}$. Reactions were sealed with rubber septa or caps and maintained in an inert environment under $\mathrm{N}_{2}$ (passed through calcium sulfate). Solid reagents were measured on a Mettler AK160 or AG104 balance. Air and moisture sensitive liquids were transferred via dry syringes. Reaction temperatures refer to the temperature that the reaction glassware was immersed. Room temperature is generally between $23-25^{\circ} \mathrm{C}$. Higher temperatures, unless otherwise indicated, were achieved by the use of a silicon oil bath heated by a nichrome wire under constant voltage. Temperatures of $0{ }^{\circ} \mathrm{C}$ and $-78{ }^{\circ} \mathrm{C}$ were maintained by the use of an ice bath and dry ice/acetone bath respectively. The term in vacuo refers to the use of a rotary evaporator with an attached vacuum membrane pump. Tetrahydrofuran, diethyl ether, toluene, and dichloromethane were passed through alumina drying columns (Solv-tek Inc.) unless otherwise indicated. Petroleum ether, ethyl acetate, and pentane was purchased from Fisher Chemical. Deuterated solvents were purchased from Cambridge Isotope Laboratories. Deuterated chloroform was stored over anhydrous potassium carbonate. Reagents were used as purchased unless otherwise indicated.

Analytical TLC was performed using $0.25 \mathrm{~mm}$ silica gel $60 \mathrm{~F}_{254}$ coated plates from EMD Chemicals Inc. and monitored at $254 \mathrm{~nm}$. Staining was accomplished using $p$-anisaldehyde, potassium permanganate, or cerium sulfate and gentle heating. Preparative flash chromatography was performed by running solvent under a pressure of air through silica gel (230-400 mech, $60 \AA$ ), purchased from Silicycle Chemical Division. Reverse phase high pressure liquid chromatography (RP-HPLC) was performed on a Varian ProStar 320 and monitored at $220 \mathrm{~nm}$. The column used was an Alltech Alltima C18 $(10 \mu \mathrm{m})$ column (length: $250 \mathrm{~mm}$, inner diamter: 10.0mm). Solvent was removed via lyophilization using a Virtis Freezemobile 25EL lyophilizer.

Optical rotations were measured using a JASCO DIP-360 digital machine. The format for measurements are: $[\alpha]_{D}^{\text {temperature }}=$ specific rotation in degrees $(c=$ concentration in $\mathrm{g} / 100 \mathrm{~mL}$, solvent). Nuclear magnetic resonance spectra were taken on a Varian Inova $600\left({ }^{1} \mathrm{H}\right.$ at 600 
MHz), Varian Inova $500\left({ }^{1} \mathrm{H}\right.$ at $\left.500 \mathrm{MHz}\right)$, or Varian Mercury $400\left({ }^{1} \mathrm{H}\right.$ at $\left.400 \mathrm{MHz}\right)$ spectrometer. ${ }^{1} \mathrm{H}$ NMR data is reported as follows: chemical shift ( $\delta$ in ppm) relative to residual solvent peak or TMS, integration, multiplicity $(\mathrm{s}=$ singlet, $\mathrm{d}=$ doublet, $\mathrm{t}=$ triplet, $\mathrm{q}=$ quartet, pent $=$ pentent, $\mathrm{dd}=$ doublet of doublets, $\mathrm{ddd}=$ doublet of doublet of doublets, dddd $=$ doublet of doublet of doublet of doublets, $\mathrm{dt}=$ doublet of triplets, $\mathrm{ddt}=$ doublet of doublet of triplets, $\mathrm{dtt}=$ doublet of triplets of triplets, $\mathrm{m}=$ multiplet), coupling constant(s), and proton assignment. Proton assignments were based on COSY experiments as well as correlation to related compounds. ${ }^{13} \mathrm{C}$ NMR data is reported as follows: chemical shift ( $\delta$ in ppm) relative to residual solvent peak or TMS. Infrared spectra were measured on a Perkin-Elmer 1600 Series Fourier transform spectrometer, are reported in wavenumbers $\left(\mathrm{cm}^{-1}\right)$, and externally referenced to polystyrene film $\left(1601 \mathrm{~cm}^{-1}\right)$. High-resolution mass spectra (HRMS) were measured at the high-resolution mass spectrometry facility at the NIH regional mass spectrometry facility at the University of California, San Francisco, the University of California, Riverside, and at the Vincent Coates foundation mass spectrometry laboratory at the Stanford University, California. Elemental analyses were measured at Desert Analytics, Tucson, Arizona. 


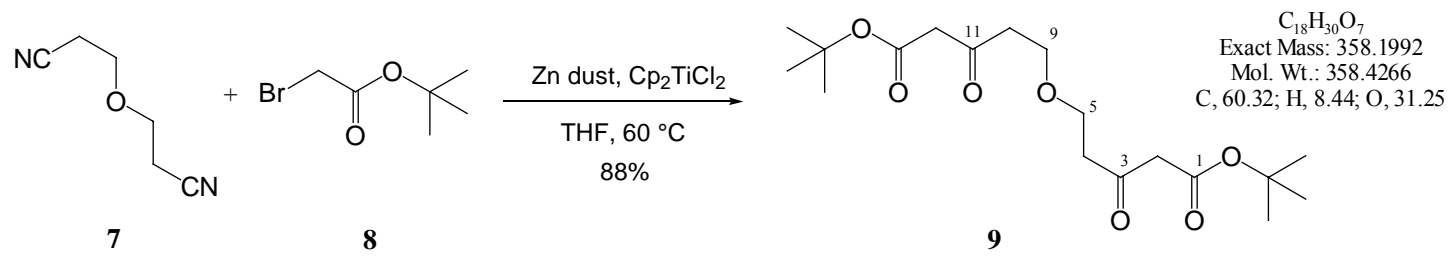

Ether $7(480 \mu \mathrm{L}, 4.032 \mathrm{mmol}), \mathrm{Zn}$ dust $(3.9 \mathrm{~g}, 60.4 \mathrm{mmol})$, and $\mathrm{Cp}_{2} \mathrm{TiCl}_{2}(30.2 \mathrm{mg}, 0.12$ mmol) were dissolved in dry THF $(20 \mathrm{~mL})$ and stirred at $60{ }^{\circ} \mathrm{C}$. Ester $8(5.4 \mathrm{~mL}, 40.0 \mathrm{mmol})$ was added over $1 \mathrm{~h}$ in $1 \mathrm{~mL}$ portions. After $4.5 \mathrm{~h}$, the reaction was filtered through celite with $\mathrm{Et}_{2} \mathrm{O}(3$ washes $\times 20 \mathrm{~mL}) .1 \mathrm{M} \mathrm{HCl}(40 \mathrm{~mL})$ was added and allowed to stir vigorously for $3 \mathrm{~h}$. The aqueous layer was extracted with $\mathrm{Et}_{2} \mathrm{O}(3 \times 50 \mathrm{~mL})$ and the combined organic layer dried over $\mathrm{Na}_{2} \mathrm{SO}_{4}$, filtered, and reduced in vacuo to a reddish oil. Flash chromatography (20\% EtOAc : pet. ether) gave 9 as a yellow oil (1.27 g, 88\%).

Data for 9:

$\mathbf{R}_{f}=0.30$ (30\% EtOAc, 70\% pentane) - one red, UV-active spot (p-anisaldehyde)

IR (film) 2979, 2930, 1713, 1642, 1369, 1317, 1257, 1149, $1043 \mathrm{~cm}^{-1}$

${ }^{1}$ H NMR (500 MHz, $\left.\mathrm{CDCl}_{3}\right) \delta 3.69(2 \mathrm{H}, \mathrm{t}, J=6.0 \mathrm{~Hz}, \mathrm{C} 5 / \mathrm{C} 9), 3.37$ (2H, s, C2 \& C12), 2.76 $(2 \mathrm{H}, \mathrm{t}, J=6.0 \mathrm{~Hz}, \mathrm{C} 4 \& \mathrm{C} 10), 1.46\left(9 \mathrm{H}, \mathrm{s},-\mathrm{O}^{t} \mathrm{Bu}\right)$ note: around $8 \%$ of enol tautomers can be seen in the ${ }^{1} \mathrm{H}$ NMR

${ }^{13}$ C NMR $\left(125 \mathrm{MHz}, \mathrm{CDCl}_{3}\right) \delta 201.7,166.3,82.0,65.7,51.0,42.8,28.0(\times 3)$

HRMS (ES ${ }^{+}$) Cacld for $\mathrm{C}_{18} \mathrm{H}_{30} \mathrm{O}_{7} \mathrm{Na}: 381.1889$ Found: 381.1887 

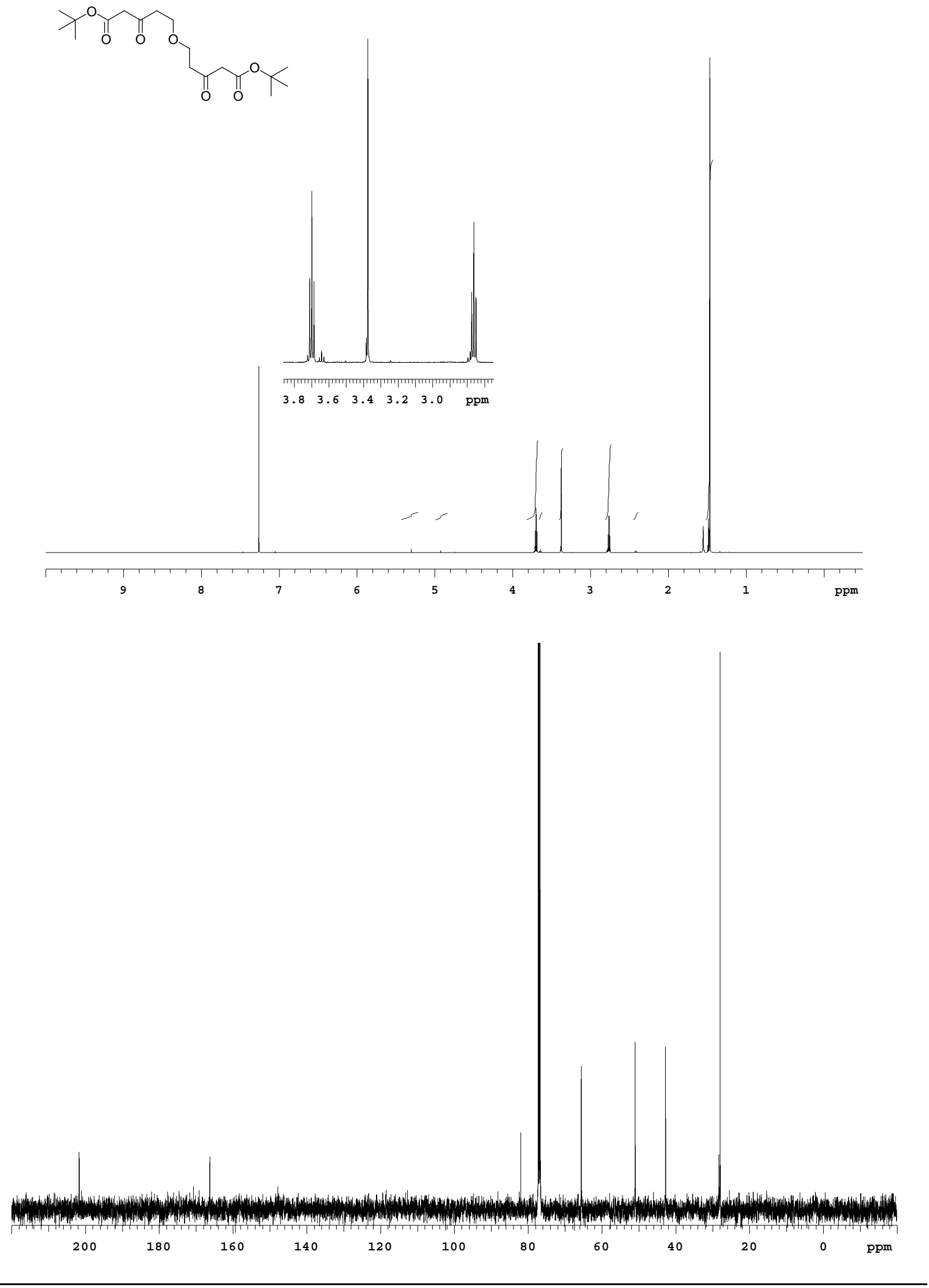


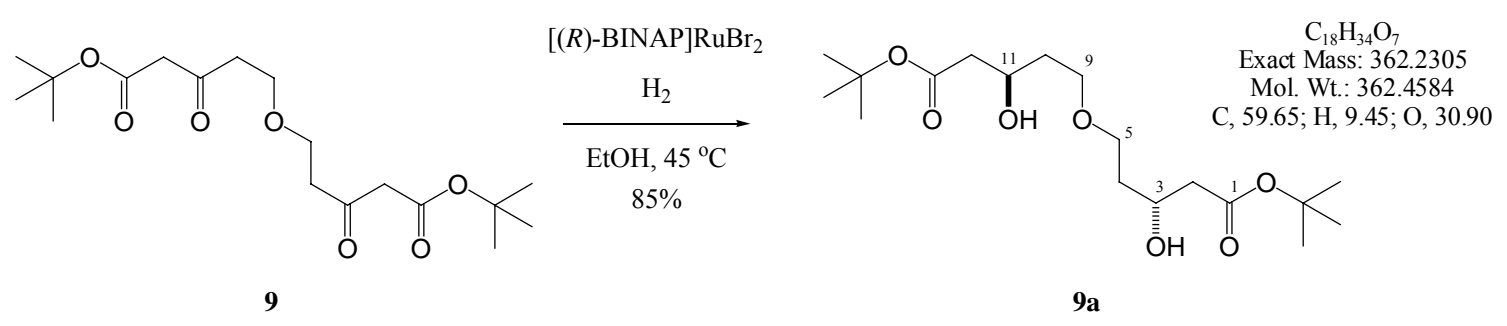

$\left[(R)\right.$-BINAP] $\mathrm{RuBr}_{2}$ was prepared by degassing acetone $(8.9 \mathrm{~mL})$ and $\mathrm{MeOH}(0.9 \mathrm{~mL})$ for $30 \mathrm{~min}$. (COD)Ru(2-methylallyl) 2 (40.4 mg, $0.1 \mathrm{mmol}$, Acros) and (R)-BINAP (95.9 mg, 0.2 mmol) was dissolved in the degassed acetone and $\mathrm{MeOH}$, followed by $\mathrm{HBr}(48 \% \mathrm{w} / \mathrm{w}, 32 \mathrm{~mL}$, $0.3 \mathrm{mmol}$ ). The solution was stirred for $1 \mathrm{~h}$, followed by low vacuum exposure for $10 \mathrm{~min}$ and by high vacuum exposure for $30 \mathrm{~min}$. The material was put under $\mathrm{N}_{2} . \mathbf{9}(912.5 \mathrm{mg}, 2.6 \mathrm{mmol})$ was dissolved in degassed $\mathrm{EtOH}(5.8 \mathrm{~mL})$ and added to the prepared [Ru] catalyst. The reaction vessel was placed in a bomb, purged $5 \mathrm{x}$ with $\mathrm{H}_{2}$, and pressurized with $\mathrm{H}_{2}$ to 40 bar. The bomb was placed in an oil bath and heated to $45{ }^{\circ} \mathrm{C}$ for $48 \mathrm{~h}$. Direct flash chromatography $(50 \%$ EtOAc : pet. ether) yielded 9a (783.8 mg, 85\%).

Data for 9a:

$\mathbf{R}_{f}=0.37$ (50\% EtOAc, 50\% pentane) - one blue spot (p-anisaldehyde)

IR (film) 3435 (br), 2978, 2930, 2873, 1722, 1393, 1367, 1293, 1257, 1152, $1112 \mathrm{~cm}^{-1}$

${ }^{1} \mathbf{H}$ NMR $\left(400 \mathrm{MHz}, \mathrm{CDCl}_{3}\right) \delta 4.12(2 \mathrm{H}, \mathrm{m}, \mathrm{C} 3 \& \mathrm{C} 11), 3.63(2 \mathrm{H}, \mathrm{m}, \mathrm{C} 5 / \mathrm{C} 9), 3.57(2 \mathrm{H}, \mathrm{m}$, C5/C9), 3.41 (2H, s (br), -OH), 2.39 (4H, m, C2 \& C12), 1.72 (4H, m, C4 \& C10), $1.45(18 \mathrm{H}, \mathrm{s}$, $\left.-\mathrm{O}^{t} \mathrm{Bu}\right)$

${ }^{13}$ C NMR $\left(100 \mathrm{MHz}, \mathrm{CDCl}_{3}\right) \delta 172.3,81.4,68.7,66.8,42.8,36.2,28.4(x 3)$

HRMS $\left(\right.$ EI $^{+}$) Cacld for $\mathrm{C}_{18} \mathrm{H}_{34} \mathrm{O}_{7} \mathrm{H}: 363.2383$ Found: 363.2375

$[\alpha]_{D}^{27}=-11.0^{\circ}\left(c=3.5, \mathrm{CHCl}_{3}\right)$

Mosher's ester analysis:
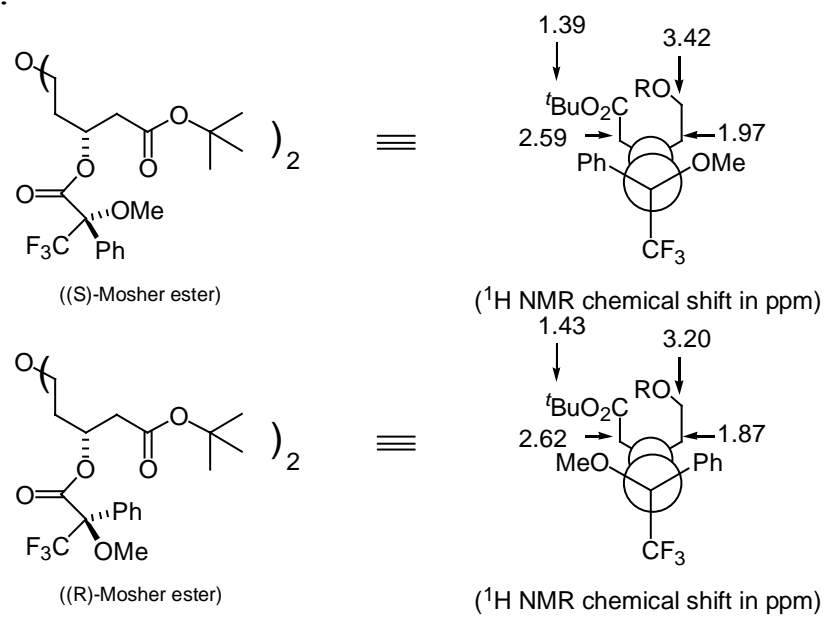

$\left({ }^{1} \mathrm{H}\right.$ NMR chemical shift in ppm) 

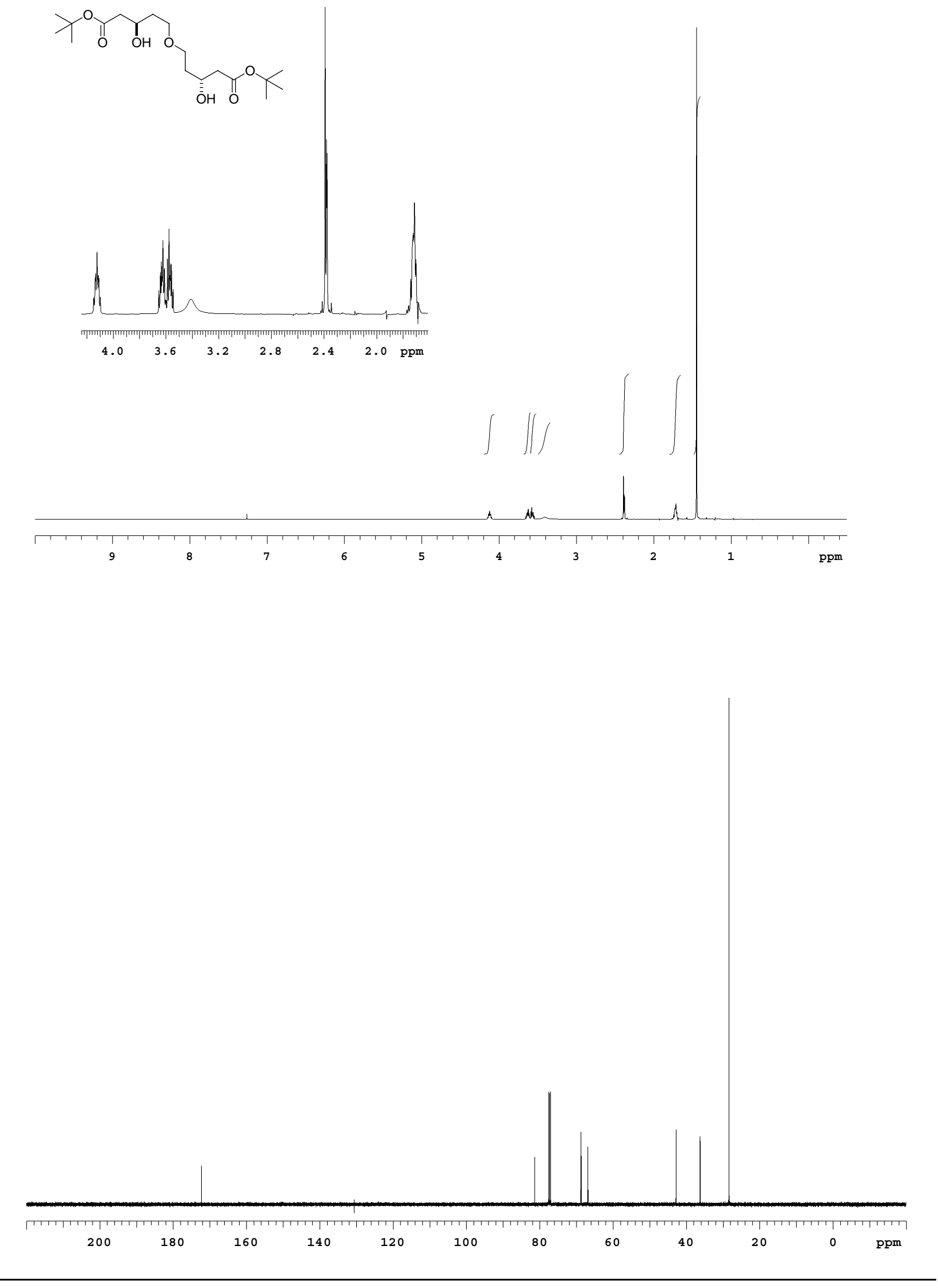


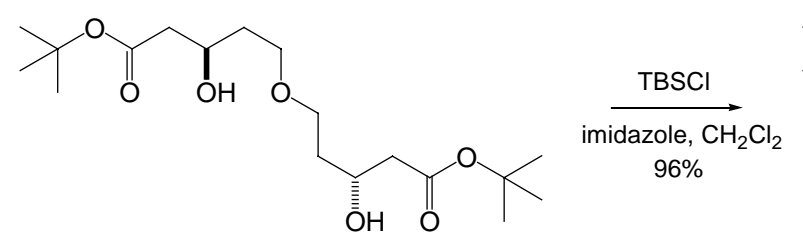

9a

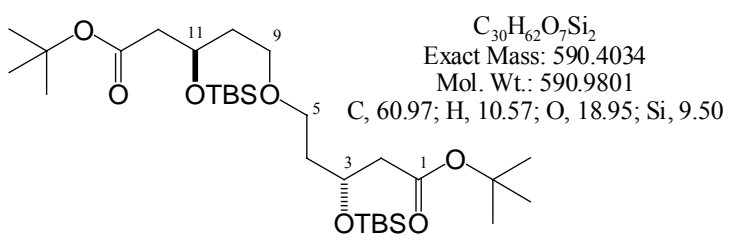

10

Diol 9a (741.0 mg, $2.04 \mathrm{mmol})$ was dissolved in $\mathrm{CH}_{2} \mathrm{Cl}_{2}(38 \mathrm{~mL})$, and imidazole $(1.4 \mathrm{~g}$, $20.8 \mathrm{mmol})$, then TBSCl $(3.1 \mathrm{~g}, 20.5 \mathrm{mmol})$ was added and the reaction was allowed to stir at $\mathrm{rt}$ for $24 \mathrm{~h}$. The reaction was quenched with satd. aq. $\mathrm{NH}_{4} \mathrm{Cl}(100 \mathrm{~mL})$ and extracted with $\mathrm{CH}_{2} \mathrm{Cl}_{2}$ $(3 \times 40 \mathrm{~mL})$. The combined organic layer was washed with brine $(50 \mathrm{~mL})$, dried over $\mathrm{MgSO}_{4}$, filtered and reduced in vacuo. Flash chromatography $(5 \rightarrow 10 \%$ EtOAc : pet. ether) yielded 10 as a clear oil (1159.0 $\mathrm{mg}, 96 \%)$.

Data for 10:

$\mathbf{R}_{f}=0.47$ (10\% EtOAc, $90 \%$ pentane) - one black spot (p-anisaldehyde)

IR (film) 2955, 2929, 2886, 2857, 1738, 1732, 1472, 1463, 1392, 1318, 1254, 1157, 1109, 1036, $1005 \mathrm{~cm}^{-1}$

${ }^{1} \mathbf{H}$ NMR $\left(400 \mathrm{MHz}, \mathrm{CDCl}_{3}\right) \delta 4.18(2 \mathrm{H}, \mathrm{m}, \mathrm{C} 3 \& \mathrm{C} 11), 3.41$ (4H, m, C5 \& C9), 2.38 (2H, dd, J $=8.0,18.5 \mathrm{~Hz}, \mathrm{C} 2$ or $\mathrm{C} 12), 2.33(2 \mathrm{H}, \mathrm{dd}, J=8.0,18.5 \mathrm{~Hz}, \mathrm{C} 12$ or $\mathrm{C} 2), 1.74(4 \mathrm{H}, \mathrm{m}, \mathrm{C} 4$ \& $\mathrm{C} 10)$, $1.41\left(18 \mathrm{H}, \mathrm{s},-\mathrm{O}^{t} \mathrm{Bu}\right), 0.84\left(18 \mathrm{H}, \mathrm{s},-\mathrm{SiO}^{t} \mathrm{Bu}\right), 0.036(6 \mathrm{H}, \mathrm{s},-\mathrm{SiMe}), 0.034(6 \mathrm{H}, \mathrm{s},-\mathrm{SiMe})$

${ }^{13} \mathrm{C}$ NMR $\left(100 \mathrm{MHz}, \mathrm{CDCl}_{3}\right) \delta 170.7,80.2,67.0,66.7,44.0,37.2,28.1(\mathrm{x} 3), 25.8(\mathrm{x} 3), 18.0,-$ $4.7,-4.8$

HRMS (ES ${ }^{+}$) Cacld for $\mathrm{C}_{30} \mathrm{H}_{62} \mathrm{O}_{7} \mathrm{Si}_{2} \mathrm{Na}$ : 613.3932 Found: 613.3936

$[\alpha]_{D}^{27}=-2.1^{\circ}\left(c=23.5, \mathrm{CDCl}_{3}\right)$

Anal. calcd for $\mathrm{C}_{30} \mathrm{H}_{62} \mathrm{O}_{7} \mathrm{Si}_{2}: \mathrm{C}, 60.97 ; \mathrm{H}, 10.57$. Found: $\mathrm{C}, 60.90 ; \mathrm{H}, 10.38$ 

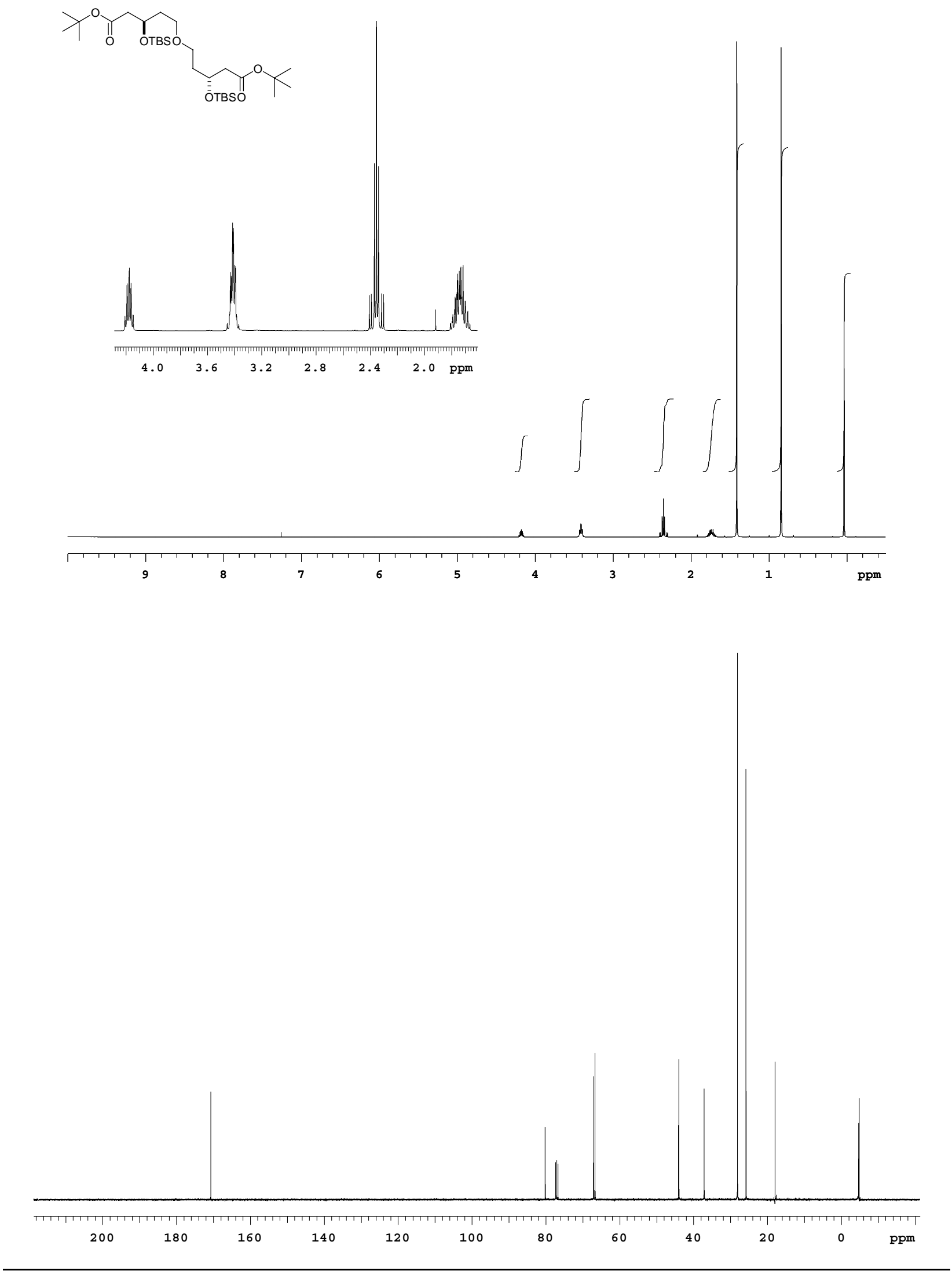


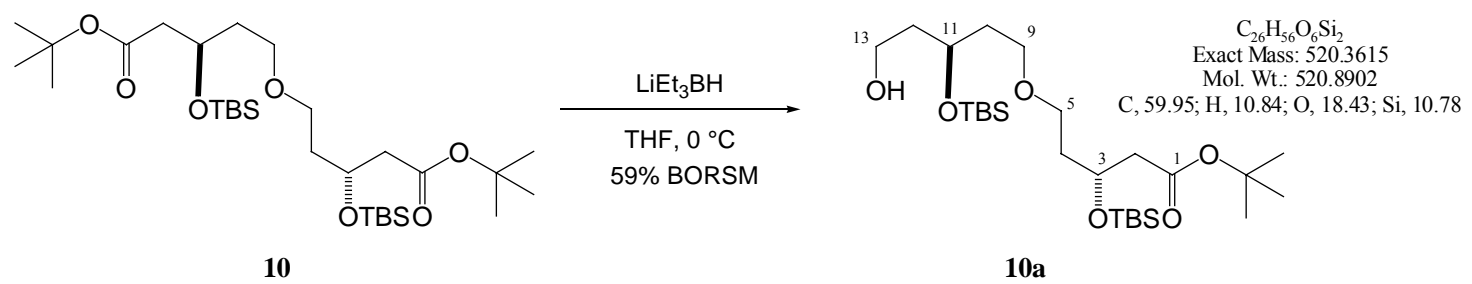

Diol $10(307.7 \mathrm{mg}, 0.521 \mathrm{mmol})$ was dissolved in THF $(9 \mathrm{~mL})$ and cooled to $0{ }^{\circ} \mathrm{C}$. Super Hydride (1.05 mL, 1.0M in THF, $1.05 \mathrm{mmol}$ ) was added dropwise over $2 \mathrm{~min}$. The reaction was allowed to stir for $1.75 \mathrm{~h}$, followed by quenching with satd. aq. $\mathrm{NH}_{4} \mathrm{Cl}(50 \mathrm{~mL})$. The solution was extracted with $\mathrm{Et}_{2} \mathrm{O}(3 \times 40 \mathrm{~mL})$ and the combined organic layer dried over $\mathrm{MgSO}_{4}$, filtered, and reduced in vacuo. Flash chromatography $(10 \rightarrow 20 \%$ EtOAc : pet. ether $)$ gave alcohol 10a (106.7 mg, 59\% BORSM) and starting material 10 (104.0 mg).

Data for 10a:

$\mathbf{R}_{f}=0.39$ (20\% EtOAc, $80 \%$ pentane $)-$ one blue spot (p-anisaldehyde)

IR (film) 3452 (br), 2955, 2929, 2885, 2857, 1732, 1463, 1472, 1368, 1318, 1256, 1157, 1109 , $1037,1005 \mathrm{~cm}^{-1}$

${ }^{1}$ H NMR $\left(500 \mathrm{MHz}, \mathrm{CDCl}_{3}\right) \delta 4.20(1 \mathrm{H}, \mathrm{ddt}, J=6.5,6.5,11.5 \mathrm{~Hz}, \mathrm{C} 3), 4.07(1 \mathrm{H}, \mathrm{ddt}, J=6.0$, 6.0, $11.0 \mathrm{~Hz}, \mathrm{C} 11), 3.83(1 \mathrm{H}, \mathrm{m}, \mathrm{C} 13), 3.72(1 \mathrm{H}, \mathrm{m}, \mathrm{C} 13), 3.44(4 \mathrm{H}, \mathrm{m}, \mathrm{C} 5$ \& C9), $2.49(1 \mathrm{H}, \mathrm{t}$, $5.5 \mathrm{~Hz},-\mathrm{OH}), 2.41(1 \mathrm{H}, \mathrm{dd}, J=6.0,14.5 \mathrm{~Hz}, \mathrm{C} 2), 2.36(1 \mathrm{H}, \mathrm{dd}, J=6.0,14.5 \mathrm{~Hz}, \mathrm{C} 2), 1.87(2 \mathrm{H}$, $\mathrm{m}, \mathrm{C} 10$ \& $\mathrm{C} 12), 1.78(2 \mathrm{H}, \mathrm{m}, \mathrm{C} 4), 1.68(2 \mathrm{H}, \mathrm{m}, \mathrm{C} 10 \& \mathrm{C} 12), 1.44\left(9 \mathrm{H}, \mathrm{s},-\mathrm{O}^{t} \mathrm{Bu}\right), 0.89(9 \mathrm{H}, \mathrm{s},-$ $\left.\mathrm{Si}{ }^{t} \mathrm{Bu}\right), 0.87$ (9H, s, $\left.-\mathrm{Si}{ }^{t} \mathrm{Bu}\right), 0.10$ (3H, s, -SiMe), 0.07 (3H, s, -SiMe), $0.06(6 \mathrm{H}, \mathrm{s},-\mathrm{SiMe})$

${ }^{13}$ C NMR $\left(125 \mathrm{MHz}, \mathrm{CDCl}_{3}\right) \delta 170.8,80.3,68.9,67.2,67.1,66.7,59.9,44.0,33.4,37.1,36.7$, 28.1 (x3), 25.8 (x6), 18.0, 17.9, -4.7, -4.7, $-4.7,-4.8$

HRMS (ES ${ }^{+}$) Cacld for $\mathrm{C}_{26} \mathrm{H}_{56} \mathrm{O}_{6} \mathrm{Si}_{2} \mathrm{Na}: 543.3513$ Found: 543.3521

$[\alpha]_{D}^{27}=-2.1^{\circ}\left(c=9.6, \mathrm{CDCl}_{3}\right)$ 

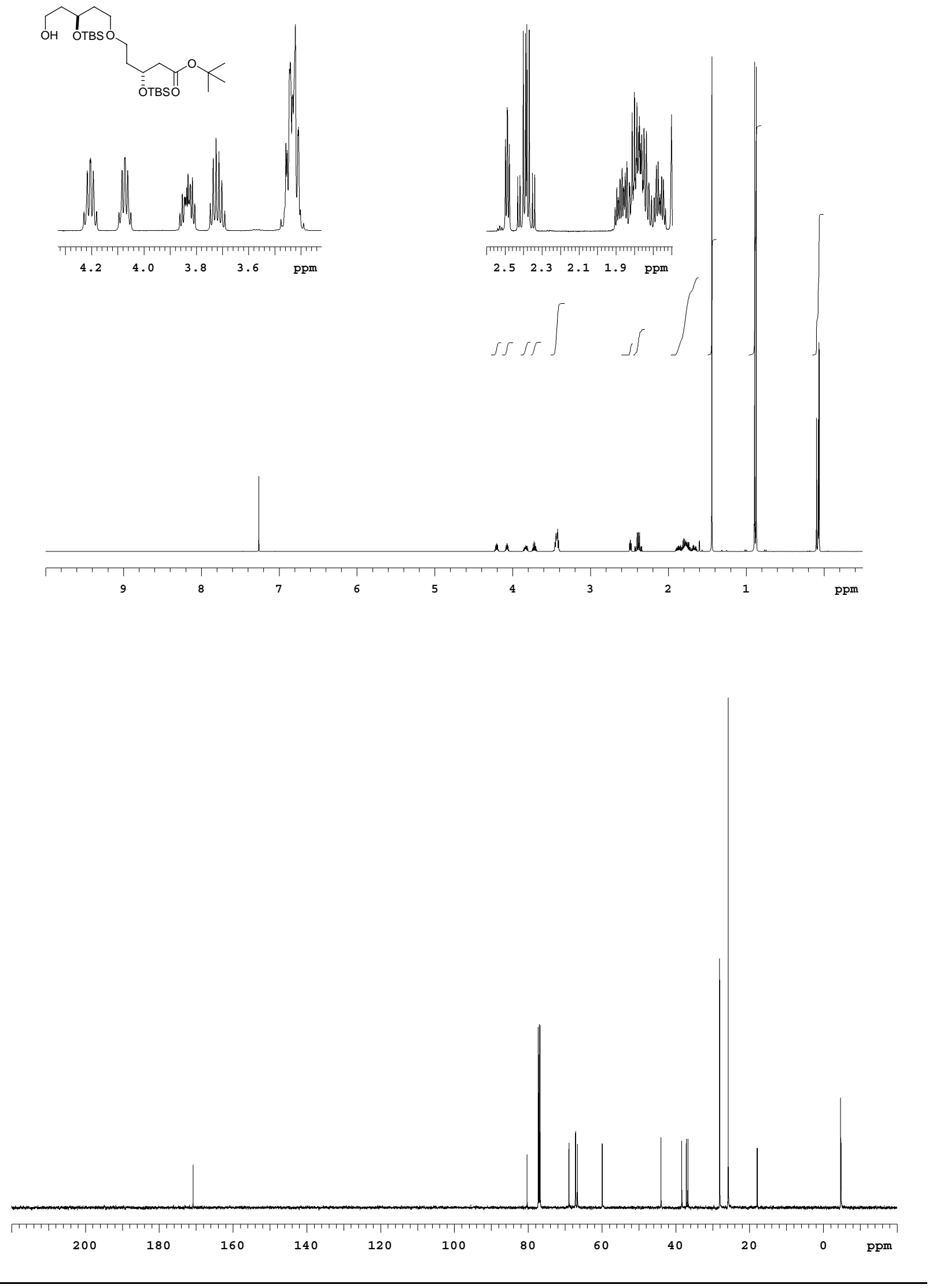

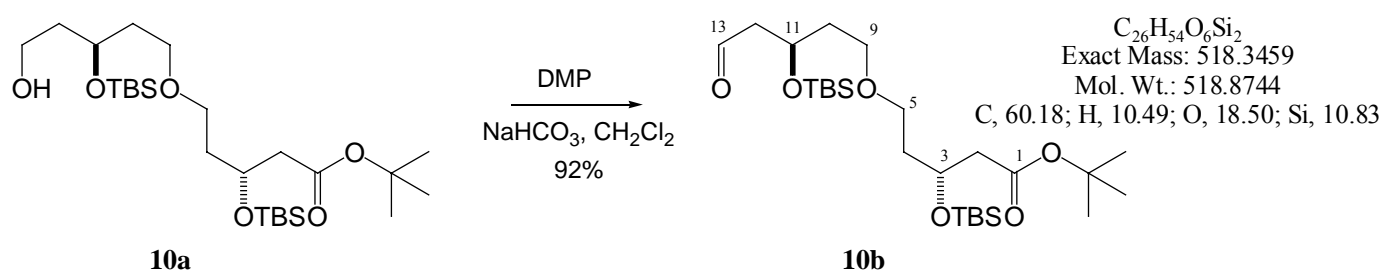

Alcohol 10a (12.0 mg, $0.023 \mathrm{mmol})$ was dissolved in $\mathrm{CH}_{2} \mathrm{Cl}_{2}(8 \mathrm{~mL}) . \mathrm{NaHCO}_{3}(5.4 \mathrm{mg}$, $0.064 \mathrm{mmol}$ ) and Dess-Martin Periodinane $(19.6 \mathrm{mg}, 0.046 \mathrm{mmol})$ was added and the reaction was allowed to stir 10 min at rt. The reaction was quenched with 1:1 satd. aq. $\mathrm{Na}_{2} \mathrm{~S}_{2} \mathrm{O}_{3}: \mathrm{NaHCO}_{3}$ $(1 \mathrm{~mL})$ and $\mathrm{H}_{2} \mathrm{O}(0.5 \mathrm{~mL})$ and stirred until the organic layer became clear. The solution was extracted with $\mathrm{CH}_{2} \mathrm{Cl}_{2}(3 \times 2 \mathrm{~mL})$, dried over $\mathrm{MgSO}_{4}$, filtered and reduced in vacuo. Flash chromatography (10\% EtOAc : pentane) gave pure $\mathbf{1 0 b}(11.0 \mathrm{mg}, 92 \%)$ as an oil.

Data for 10b:

$\mathbf{R}_{f}=0.61$ (10\% EtOAc, 90\% pentane) - one red spot (p-anisaldehyde)

IR (film) 2955, 2929, 2986, 2856, 1729, 1463, 1473, 1390, 1368, 1255, 1157, 1112, 1036, 1006 $\mathrm{cm}^{-1}$

${ }^{1} \mathbf{H}$ NMR $\left(500 \mathrm{MHz}, \mathrm{CDCl}_{3}\right) \delta 9.80(1 \mathrm{H}, \mathrm{t}, J=2.3 \mathrm{~Hz}, \mathrm{C} 13), 4.35(1 \mathrm{H}$, pent, $J=5.5 \mathrm{~Hz}, \mathrm{C} 11)$, $4.20(1 \mathrm{H}$, pent, $J=5.5 \mathrm{~Hz}, \mathrm{C} 3), 3.44$ (4H, m, C5 \& C9), 2.59 (1H, ddd, $J=2.3,5.0,15.5 \mathrm{~Hz}$, C12), 2.52 (1H, ddd, $J=2.3,6.0,15.5 \mathrm{~Hz}, \mathrm{C} 12), 2.41(1 \mathrm{H}, \mathrm{dd}, J=6.0,15.0 \mathrm{~Hz}, \mathrm{C} 2), 2.35(1 \mathrm{H}$, $\mathrm{dd}, J=6.0,15.0 \mathrm{~Hz}, \mathrm{C} 2), 1.76(4 \mathrm{H}, \mathrm{m}, \mathrm{C} 6 \& \mathrm{C} 10), 1.44\left(9 \mathrm{H}, \mathrm{s},-\mathrm{O}^{t} \mathrm{Bu}\right), 0.87\left(18 \mathrm{H}, \mathrm{s},-\mathrm{Si}{ }^{t} \mathrm{Bu}\right)$, 0.07 (6H, s, -SiMe), 0.06 (6H, s, -SiMe)

${ }^{13} \mathrm{C}$ NMR $\left(125 \mathrm{MHz}, \mathrm{CDCl}_{3}\right) \delta 202.1,170.7,80.3,67.1,66.7,66.6,65.6,51.1,44.0,37.6,37.1$, 28.1 (x3), 25.8 (x3), 25.7 (x3), 18.0, 17.9, -4.7, -4.7, -4.7, -4.8

HRMS (ES ${ }^{+}$) Cacld for $\mathrm{C}_{26} \mathrm{H}_{54} \mathrm{O}_{6} \mathrm{Si}_{2} \mathrm{Na}$ : 541.3357 Found: 541.3365

$[\alpha]_{D}^{27}=6.5^{\circ}\left(c=1.5, \mathrm{CHCl}_{3}\right)$ 

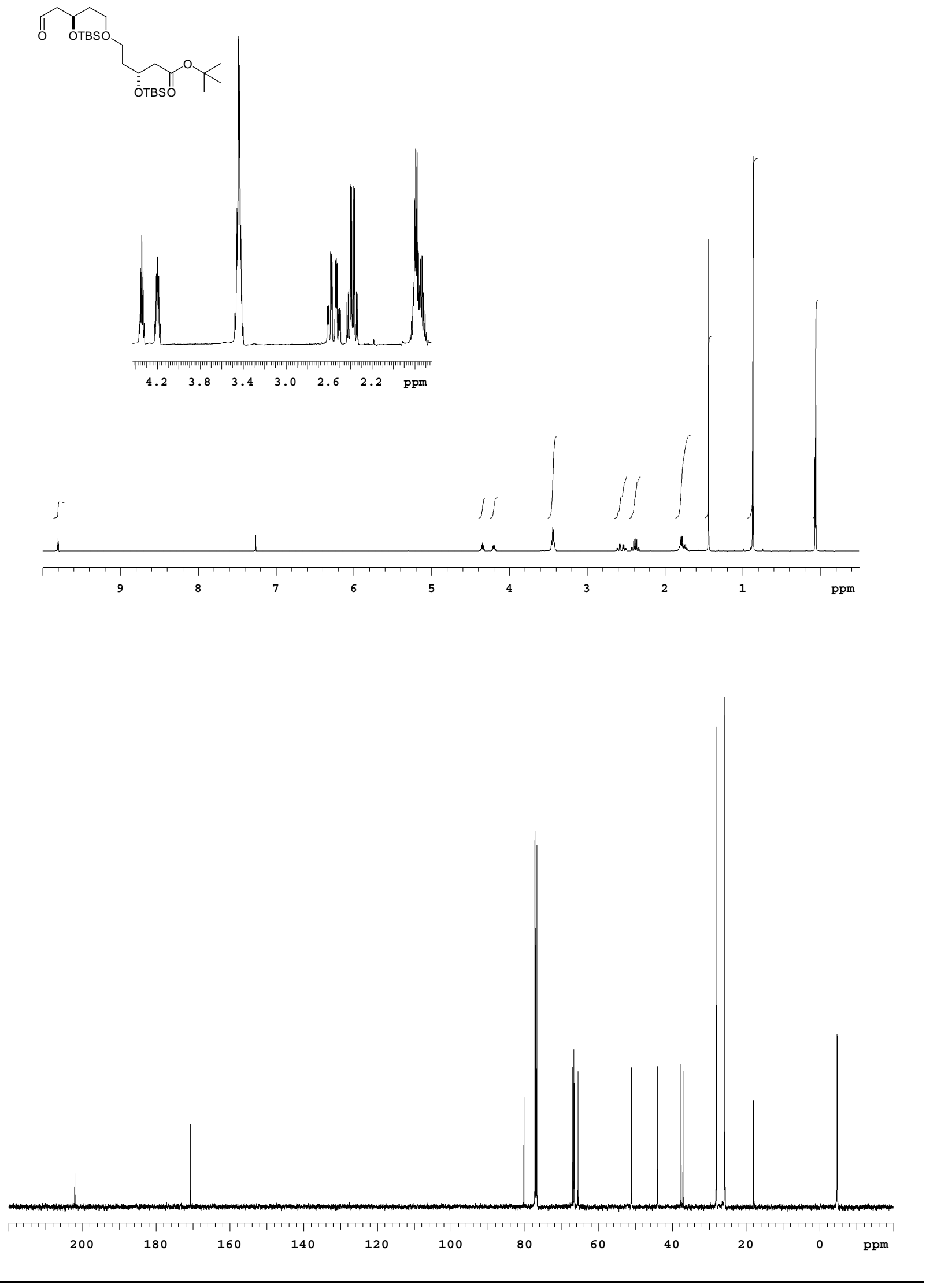


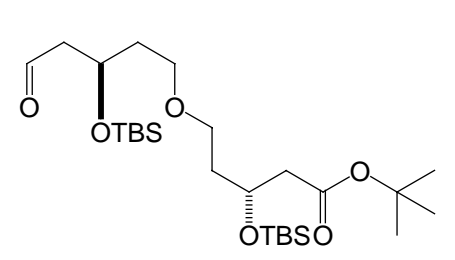

10b

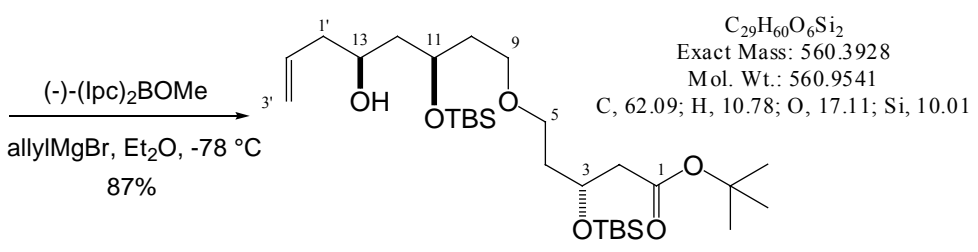

10c

(-)-(IPC) $)_{2} \mathrm{BOMe}(71.1 \mathrm{mg}, 0.202 \mathrm{mmol})$ was weighed out into a flask in a drybox. $\mathrm{Et}_{2} \mathrm{O}$ $(830 \mu \mathrm{L})$ was added and the solution cooled to $-78^{\circ} \mathrm{C}$. AllylMgBr $\left(180 \mu \mathrm{L}, 0.9 \mathrm{M}\right.$ in $\mathrm{Et}_{2} \mathrm{O}$, $0.162 \mathrm{mmol}$ ) was added and the reaction stirred first for $5 \mathrm{~min}$ at $-78^{\circ} \mathrm{C}$, then for $1 \mathrm{~h} \mathrm{at} \mathrm{rt}$. The reaction was recooled to $-78{ }^{\circ} \mathrm{C}$ and $10 \mathrm{~b}(57.5 \mathrm{mg}, 0.111 \mathrm{mmol})$ was added in $\mathrm{Et}_{2} \mathrm{O}(2 \mathrm{~mL})$ and allowed to stir for $2.5 \mathrm{~h}$. The reaction was quenched with $\mathrm{H}_{2} \mathrm{O}_{2}(30 \%, 2.6 \mathrm{~mL})$ and $1 \mathrm{~N} \mathrm{NaOH}$ $(500 \mu \mathrm{L})$ and stirred at $30{ }^{\circ} \mathrm{C}$ for $30 \mathrm{~min}$. The solution was then extracted with $\mathrm{Et}_{2} \mathrm{O}(3 \times 10 \mathrm{~mL})$ and the combined organic layer dried over $\mathrm{MgSO}_{4}$, filtered and reduced in vacuo. The crude material was dissolved in $\mathrm{MeOH}$ and evaporated in vacuo $(3 \times 2 \mathrm{~mL})$ to remove the remaining borane. Flash chromatography (10\% EtOAc : pet. ether) gave 10c $(54.0 \mathrm{mg}, 87 \%)$ as an oil.

Data for 10c:

$\mathbf{R}_{f}=0.56(10 \%$ EtOAc, $90 \%$ pentane $)-$ one black spot (p-anisaldehyde)

IR (film) 3467 (br), 2954, 2929, 2886, 2857, 1731, 1641, 1473, 1463, 1368, 1255, 1157, 1107, $1032,1005 \mathrm{~cm}^{-1}$

${ }^{1} \mathbf{H}$ NMR $\left(500 \mathrm{MHz}, \mathrm{CDCl}_{3}\right) \delta 5.83\left(1 \mathrm{H}\right.$, dddd, $\left.J=7.0,7.0,10.5,17.5, \mathrm{C}^{\prime}\right), 5.11\left(2 \mathrm{H}, \mathrm{m}, \mathrm{C}^{\prime}\right)$, $4.20(1 \mathrm{H}, \mathrm{ddt}, J=6.5,6.5,11.5 \mathrm{~Hz}, \mathrm{C} 3), 4.05(1 \mathrm{H}, \mathrm{m}, \mathrm{C} 11), 3.82(1 \mathrm{H}, \mathrm{m}, \mathrm{C} 13), 3.43(4 \mathrm{H}, \mathrm{m}, \mathrm{C} 5$ \& C9), $3.04(1 \mathrm{H}, \mathrm{d}, J=1.9 \mathrm{~Hz},-\mathrm{OH}), 2.41(1 \mathrm{H}, \mathrm{dd}, J=6.5,15.0 \mathrm{~Hz}, \mathrm{C} 2), 2.36(1 \mathrm{H}, \mathrm{dd}, J=6.5$, $15.0 \mathrm{~Hz}, \mathrm{C} 2), 2.22\left(2 \mathrm{H}, \mathrm{t}, J=7.0 \mathrm{~Hz}, \mathrm{C}^{\prime}\right), 1.77(2 \mathrm{H}, \mathrm{m}, \mathrm{C} 4 \& \mathrm{C} 10), 1.67(1 \mathrm{H}, \mathrm{ddd}, J=2.6,4.9$, $7.5 \mathrm{~Hz}, \mathrm{C} 4 / \mathrm{C} 10), 1.56(2 \mathrm{H}, \mathrm{m}, \mathrm{C} 12), 1.44\left(9 \mathrm{H}, \mathrm{s},-\mathrm{O}^{t} \mathrm{Bu}\right), 0.89\left(9 \mathrm{H}, \mathrm{s},-\mathrm{Si}{ }^{t} \mathrm{Bu}\right), 0.87(9 \mathrm{H}, \mathrm{s},-$ $\left.\mathrm{Si}^{t} \mathrm{Bu}\right), 0.11(2 \mathrm{H}, \mathrm{s},-\mathrm{SiMe}), 0.10(2 \mathrm{H}, \mathrm{s},-\mathrm{SiMe}), 0.06(4 \mathrm{H}, \mathrm{s},-\mathrm{SiMe})$

${ }^{13}$ C NMR $\left(125 \mathrm{MHz}, \mathrm{CDCl}_{3}\right) \delta 170.8,134.9,117.5,80.3,70.1,69.6,67.2,67.1,66.7,44.0,42.9$, $42.2,37.8,37.1,28.1(\mathrm{x} 3), 25.8(\mathrm{x} 6), 18.0,17.9,-4.3,-4.7(\mathrm{x} 2),-4.8$

HRMS (ES ${ }^{+}$) Cacld for $\mathrm{C}_{29} \mathrm{H}_{60} \mathrm{O}_{6} \mathrm{Si}_{2} \mathrm{Na}: 583.3826$ Found: 583.3820

$[\alpha]_{D}^{27}=-10.6^{\circ}\left(c=0.55, \mathrm{CHCl}_{3}\right)$ 
Mosher's ester analysis:

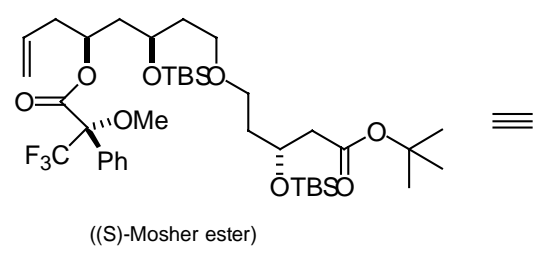

((S)-Mosher ester)

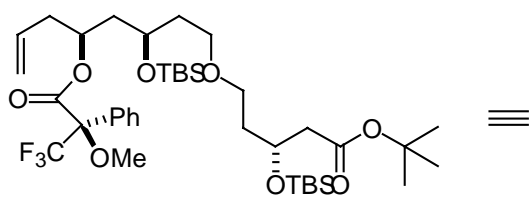

((R)-Mosher ester)

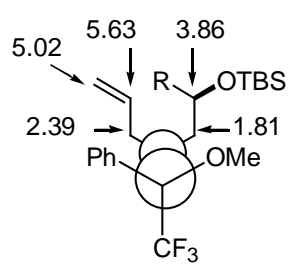

$\left({ }^{1} \mathrm{H}\right.$ NMR chemical shift in ppm)

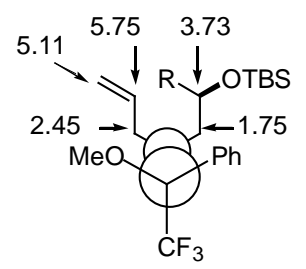

$\left({ }^{1} \mathrm{H}\right.$ NMR chemical shift in ppm) 

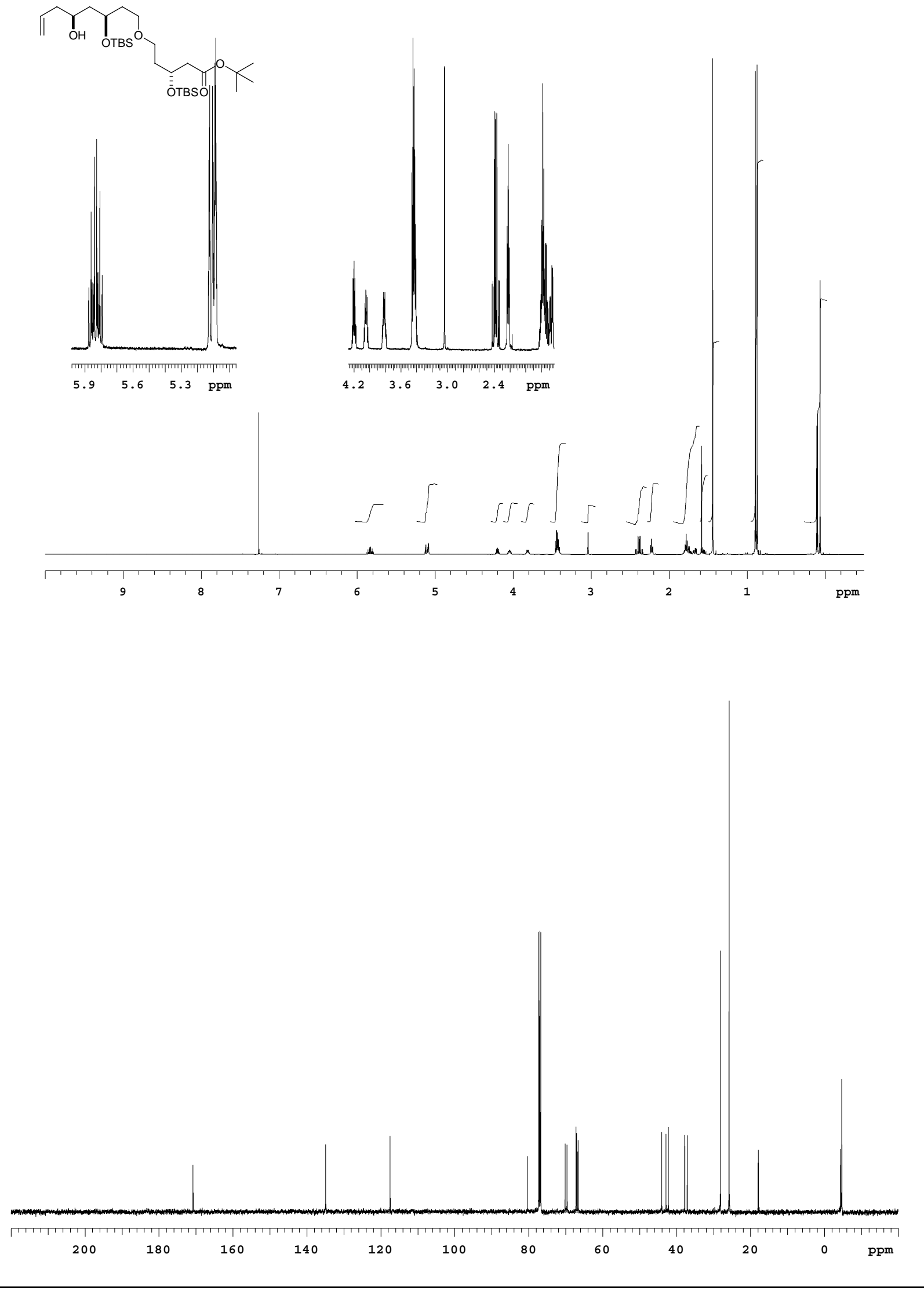


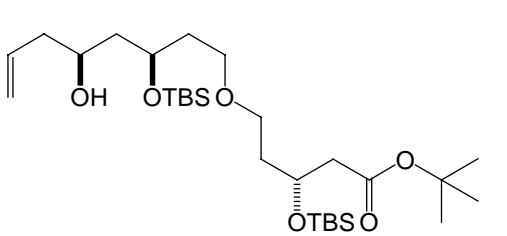

10c

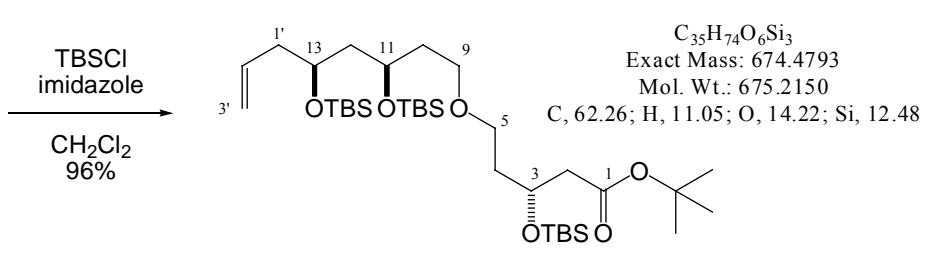

11

Alcohol 10c (50.8 mg, $0.091 \mathrm{mmol})$ was dissolved in $\mathrm{CH}_{2} \mathrm{Cl}_{2}(1.5 \mathrm{~mL})$. Imidazole $(61.5$ $\mathrm{mg}, 0.903 \mathrm{mmol})$, followed by TBSCl $(140.5 \mathrm{mg}, 0.930 \mathrm{mmol})$ was added and the reaction stirred at ambient temperature for $7 \mathrm{~h}$. The reaction was quenched with satd. aq. $\mathrm{NH}_{4} \mathrm{Cl}(4 \mathrm{~mL})$ and extracted with $\mathrm{CH}_{2} \mathrm{Cl}_{2}(3 \times 7 \mathrm{~mL})$. The combined organic layers were dried over $\mathrm{Na}_{2} \mathrm{SO}_{4}$, filtered, and reduced in vacuo. Flash chromatography (5\% EtOAc : pentane) gave 11 as a clear oil (59.2 mg, 96\%).

Data for 11:

$\mathbf{R}_{f}=0.56(10 \%$ EtOAc, $90 \%$ pentane $)$ - one black spot (p-anisaldehyde)

IR (film) 2956, 2929, 2886, 2857, 1737, 1733, 1641, 1472, 1463, 1386, 1367, 1256, 1157, 1112 , $1005 \mathrm{~cm}^{-1}$

${ }^{1} \mathbf{H}$ NMR $\left(600 \mathrm{MHz}, \mathrm{CDCl}_{3}\right) \delta 5.80(1 \mathrm{H}, \mathrm{m}, \mathrm{C} 2$ ') $5.03(2 \mathrm{H}, \mathrm{m}, \mathrm{C} 3$ ') $, 4.20(1 \mathrm{H}, \mathrm{m}, \mathrm{C} 3), 3.89(1 \mathrm{H}$, m, C11), 3.79 (1H, m, C13), $3.43(4 \mathrm{H}, \mathrm{m}, \mathrm{C} 5 \& \mathrm{C} 9), 2.40(1 \mathrm{H}, \mathrm{dd}, J=6.6,14.4 \mathrm{~Hz}, \mathrm{C} 2), 2.36$ $\left.(1 \mathrm{H}, \mathrm{dd}, J=6.6,14.4 \mathrm{~Hz}, \mathrm{C} 2), 2.26\left(1 \mathrm{H}, \mathrm{m}, \mathrm{C} 1{ }^{\prime}\right), 2.17(1 \mathrm{H}, \mathrm{m}, \mathrm{C} 1)^{\prime}\right), 1.76(3 \mathrm{H}, \mathrm{m}, \mathrm{C} 4(\mathrm{x} 2) \&$ $\mathrm{C} 10), 1.60$ (3H, m, C10 \& C12 (x2)), 1.44 (9H, s, -O $\left.{ }^{t} \mathrm{Bu}\right), 0.88\left(9 \mathrm{H}, \mathrm{s},-\mathrm{Si}{ }^{t} \mathrm{Bu}\right), 0.88(9 \mathrm{H}, \mathrm{s},-$ $\left.\mathrm{Si}^{t} \mathrm{Bu}\right), 0.87$ (9H, s, -Si $\left.{ }^{t} \mathrm{Bu}\right), 0.07$ (3H, s, -SiMe), 0.06 (3H, s, -SiMe), 0.05 (6H, s, -SiMe), 0.05 (3H, s, -SiMe), 0.04 (3H, s, -SiMe)

${ }^{13} \mathrm{C}$ NMR $\left(150 \mathrm{MHz}, \mathrm{CDCl}_{3}\right) \delta 170.8,134.9,117.0,80.2,69.0,67.4,67.2,66.8,66.8,44.9,44.1$, 42.1, 37.2, 37.1, 28.2 (x3), 25.9 (x6), 25.8 (x3), 18.0, 18.0, 18.0, -4.3, -4.4, -4.5, -4.6, -4.6, -4.8

HRMS (ES ${ }^{+}$) Cacld for $\mathrm{C}_{35} \mathrm{H}_{74} \mathrm{O}_{6} \mathrm{Si}_{3} \mathrm{Na}$ : 697.4691 Found: 697.4674

$[\alpha]_{D}^{27}=12.2^{\circ}\left(c=5.9, \mathrm{CHCl}_{3}\right)$ 

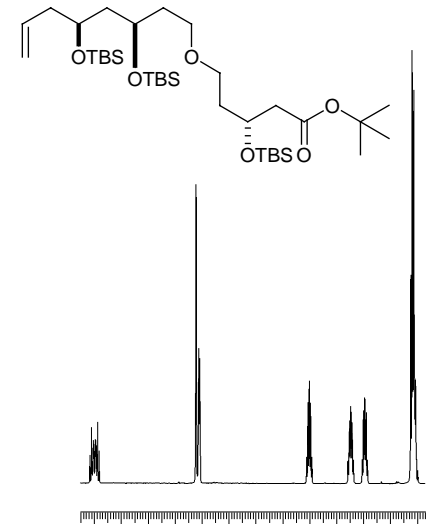
$\begin{array}{lllll}5.8 & 5.2 & 4.6 & 4.0 & \mathrm{ppm}\end{array}$

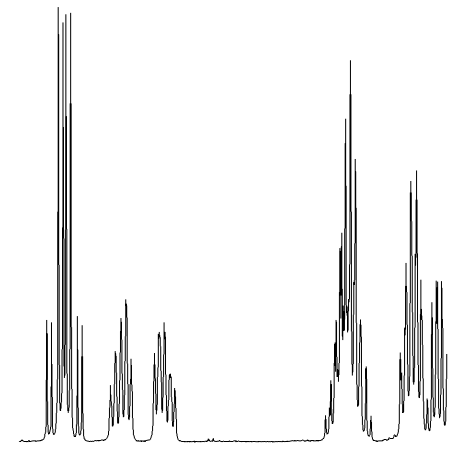

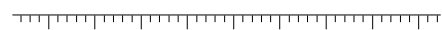
$\begin{array}{lllll}2.4 & 2.2 & 2.0 & 1.8 & \mathrm{ppm}\end{array}$
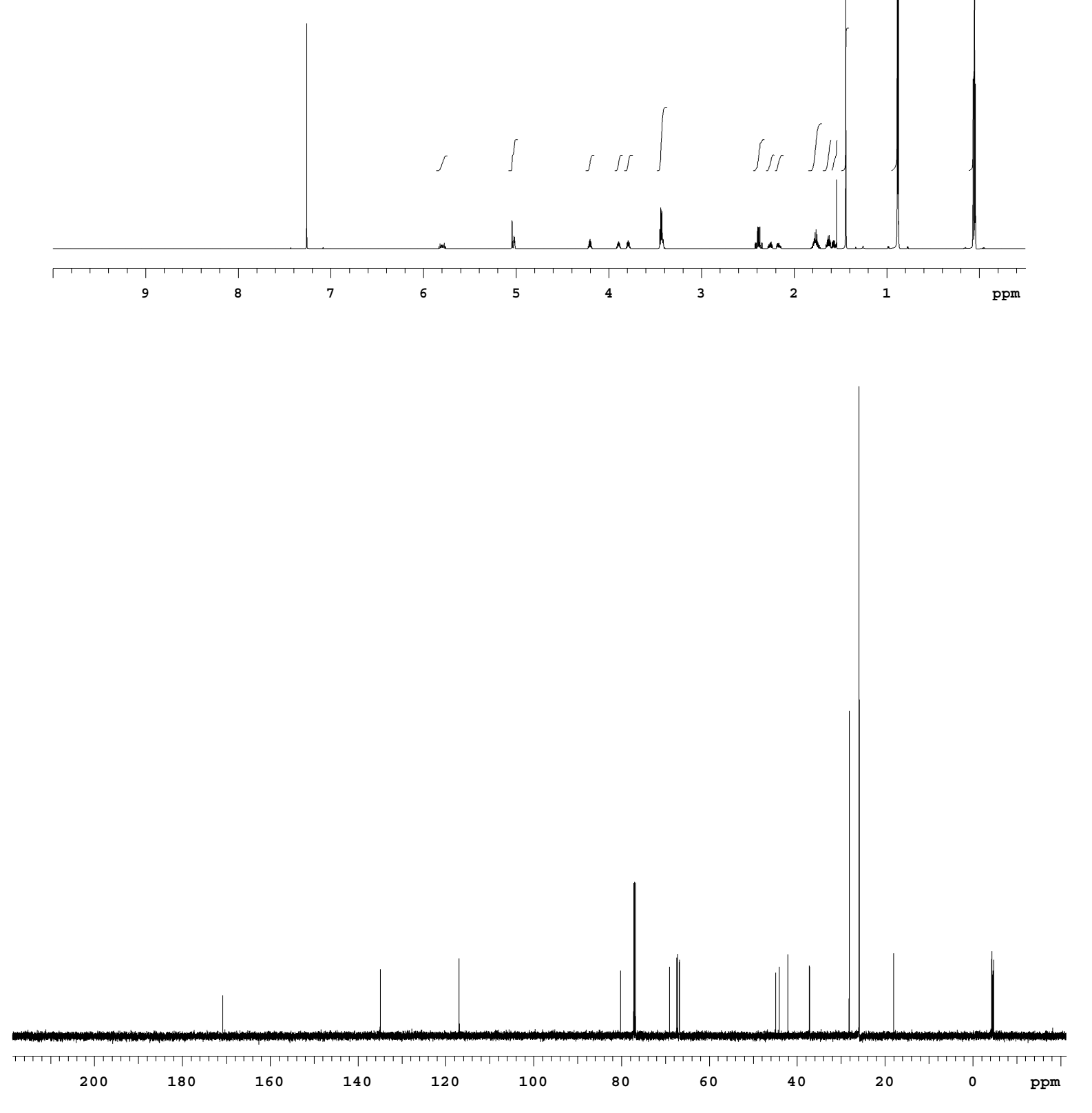


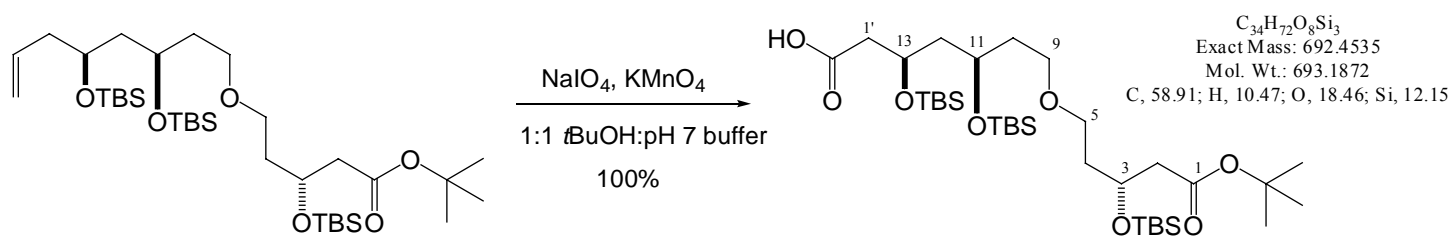

11

$11 a$

$\mathrm{NaIO}_{4}(95.4 \mathrm{mg}, 0.446 \mathrm{mmol})$ and $\mathrm{KMnO}_{4}(9.1 \mathrm{mg}, 0.058 \mathrm{mmol})$ were dissolved in $\mathrm{pH} 7$ buffer $(3 \mathrm{~mL})$ and stirred at $\mathrm{rt}$ for $15 \mathrm{~min}$. Terminal alkene $11(38.0 \mathrm{mg}, 0.056 \mathrm{mmol})$ was dissolved in ${ }^{t} \mathrm{BuOH}(2.2 \mathrm{~mL})$. The $\mathrm{NaIO}_{4} / \mathrm{KMnO}_{4}$ solution was added with an additional $\mathrm{pH} 7$ buffer wash $(1.4 \mathrm{~mL})$. The reaction was allowed to stir at $\mathrm{rt}$ for $5 \mathrm{~h}$, after which the reaction was quenched with $\mathrm{Na}_{2} \mathrm{~S}_{2} \mathrm{O}_{3} \cdot 5 \mathrm{H}_{2} \mathrm{O}$. The reaction turned brown and was allowed to stir for $5 \mathrm{~min}$. Brine $(40 \mathrm{~mL})$ was added and the solution extracted with EtOAc $(3 \times 40 \mathrm{~mL})$. The combined organic layer was dried over $\mathrm{MgSO}_{4}$, filtered, and reduced in vacuo. Flash chromatography (10 $\rightarrow 20 \%$ EtOAc : pentane $+1 \% \mathrm{AcOH})$ yielded 11a $(38.7 \mathrm{mg}, 100 \%)$ as an oil.

Data for 11a:

$\mathbf{R}_{f}=0.32(20 \%$ EtOAc, $80 \%$ pentane $)-$ one black spot (p-anisaldehyde)

IR (film) 2955, 2929, 2887, 2858, 1732, 1716, 1472, 1464, 1386, 1368, 1256, 1157, 1104, 1037, $1005 \mathrm{~cm}^{-1}$

${ }^{1} \mathbf{H}$ NMR $\left(500 \mathrm{MHz}, \mathrm{CDCl}_{3}\right) \delta 4.21(2 \mathrm{H}, \mathrm{m}, \mathrm{C} 3 \& \mathrm{C} 13), 3.88(1 \mathrm{H}$, pent, $J=6.0 \mathrm{~Hz}, \mathrm{C} 11), 3.42$ (4H, m, C5 \& C9), $2.60\left(1 \mathrm{H}, \mathrm{dd}, J=5.5,15.5 \mathrm{~Hz}, \mathrm{C} 11^{\prime}\right), 2.50\left(1 \mathrm{H}, \mathrm{dd}, J=5.5,15.5 \mathrm{~Hz}, \mathrm{C} 1{ }^{\prime}\right), 2.41$ $(1 \mathrm{H}, \mathrm{dd}, J=6.5,15.0 \mathrm{~Hz}, \mathrm{C} 2), 2.37(1 \mathrm{H}, \mathrm{dd}, J=6.5,15.0 \mathrm{~Hz}, \mathrm{C} 2), 1.74(6 \mathrm{H}, \mathrm{m}, \mathrm{C} 4 \& \mathrm{C} 10$ \& C12), $1.44\left(9 \mathrm{H}, \mathrm{s},-\mathrm{O}^{t} \mathrm{Bu}\right), 0.89\left(9 \mathrm{H}, \mathrm{s},-\mathrm{Si}^{t} \mathrm{Bu}\right), 0.88\left(9 \mathrm{H}, \mathrm{s},-\mathrm{Si}{ }^{t} \mathrm{Bu}\right), 0.87\left(9 \mathrm{H}, \mathrm{s},-\mathrm{Si}{ }^{t} \mathrm{Bu}\right), 0.11$ $(3 \mathrm{H}, \mathrm{s},-\mathrm{SiMe}), 0.10(3 \mathrm{H}, \mathrm{s},-\mathrm{SiMe}), 0.07$ (3H, s, $-\mathrm{SiMe}), 0.06$ (3H, s, $-\mathrm{SiMe}), 0.05(3 \mathrm{H}, \mathrm{s},-$ $\mathrm{SiMe}), 0.05$ (3H, s, -SiMe)

${ }^{13}$ C NMR $\left(100 \mathrm{MHz}, \mathrm{CDCl}_{3}\right) \delta 176.0,171.3,80.6,67.5,67.3,67.0,66.9,66.8,45.2,44.3,42.5$, $37.4,37.4,28.4$ (x3), 26.1 (x3), 26.1 (x3), 26.0 (x3), 18.2, 18.2, 18.1, -4.2, -4.3, -4.4, -4.4, -4.5, 4.6

HRMS (ES ${ }^{+}$) Cacld for $\mathrm{C}_{34} \mathrm{H}_{72} \mathrm{O}_{8} \mathrm{Si}_{3} \mathrm{Na}$ : 715.4433 Found: 715.4435

$[\alpha]_{D}^{27}=-0.43^{\circ}\left(c=2.8, \mathrm{CHCl}_{3}\right)$ 

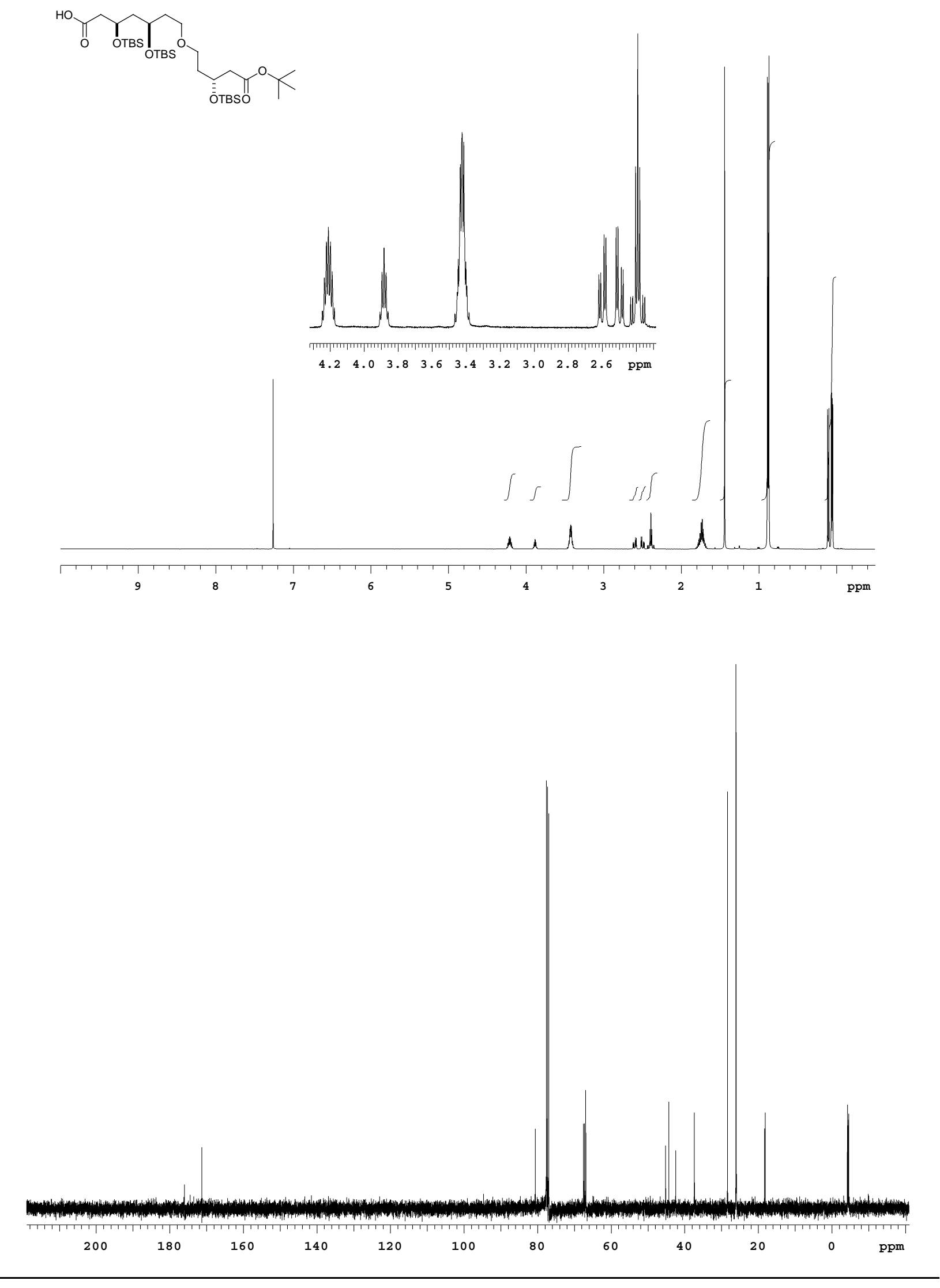


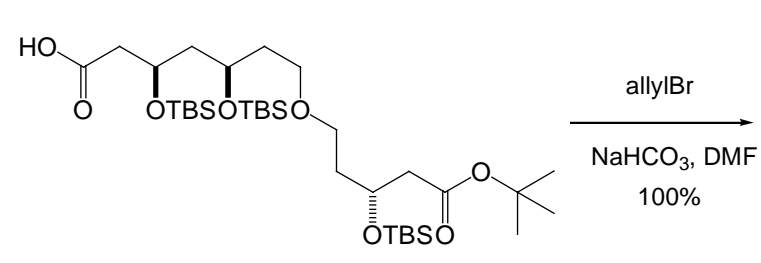

$11 \mathrm{a}$

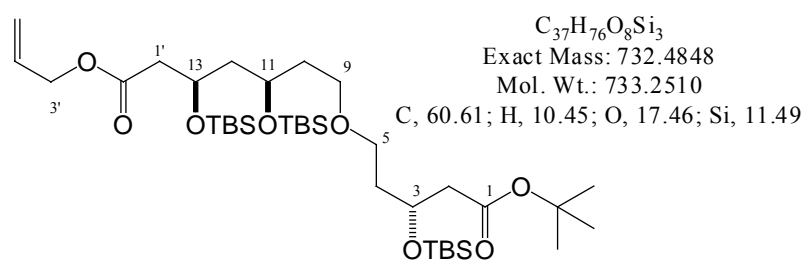

$11 b$

Acid 11a (3.1 mg, $0.005 \mathrm{mmol})$ was dissolved in DMF $(100 \mu \mathrm{L}) . \mathrm{NaHCO}_{3}$ (crystals) was added, followed by allylBr $(2 \mu \mathrm{L}, 0.02 \mathrm{mmol})$. The reaction was stirred at $\mathrm{rt}$ for $24 \mathrm{~h}$, then diluted with sat. aq. $\mathrm{NH}_{4} \mathrm{Cl}(3 \mathrm{~mL})$ and extracted with $\mathrm{Et}_{2} \mathrm{O}(3 \mathrm{x} 1 \mathrm{~mL})$, dried over $\mathrm{MgSO}_{4}$, filtered and reduced in vacuo. Flash chromatography (7\% EtOAc : pet. ether) gave $11 \mathbf{b}(3.3 \mathrm{mg}$, $100 \%$ ).

Data for 11b:

$\mathbf{R}_{f}=0.22$ (5\% EtOAc, 95\% pentane) - one black spot (p-anisaldehyde)

IR (film) 2954, 2929, 2886, 2857, 1735, 1473, 1463, 1367, 1311, 1256, 1159, 1105, 1038, 1005 $\mathrm{cm}^{-1}$

${ }^{1} \mathbf{H}$ NMR $\left(500 \mathrm{MHz}, \mathrm{CDCl}_{3}\right) \delta 5.91\left(1 \mathrm{H}, \mathrm{dddd}, J=6.0,6.0,10.5,16.5 \mathrm{~Hz}, \mathrm{C} 4{ }^{\prime}\right), 5.31\left(1 \mathrm{H}, \mathrm{m}, \mathrm{C} 5^{\prime}\right.$ (cis)), 5.23 (1H, dd, $J=1.2,10.5, \mathrm{C}^{\prime}$ (trans)), $4.56\left(2 \mathrm{H}, \mathrm{m}, \mathrm{C} 3^{\prime}\right), 4.21$ (2H, m, C3 \& C13), 3.88 (1H, pent, $J=6.0 \mathrm{~Hz}, \mathrm{C} 11), 3.42$ (4H, m, C5 \& C9), 2.55 (1H, dd, $\left.J=5.0,14.5 \mathrm{~Hz}, \mathrm{C} 1{ }^{\prime}\right), 2.43$ $\left(1 \mathrm{H}, \mathrm{dd}, J=7.5,14.5 \mathrm{~Hz}, \mathrm{C} 1^{\prime}\right), 2.40(1 \mathrm{H}, \mathrm{dd}, J=5.0,15.0 \mathrm{~Hz}, \mathrm{C} 2), 2.35(1 \mathrm{H}, \mathrm{dd}, J=6.0,15.0$, C2), $1.76(4 \mathrm{H}, \mathrm{m}, \mathrm{C} 4 \& \mathrm{C} 10), 1.64(2 \mathrm{H}, \mathrm{m}, \mathrm{C} 12), 1.44\left(9 \mathrm{H}, \mathrm{s},-\mathrm{O}^{t} \mathrm{Bu}\right), 0.88\left(9 \mathrm{H}, \mathrm{s},-\mathrm{Si}{ }^{t} \mathrm{Bu}\right), 0.87$ $\left(9 \mathrm{H}, \mathrm{s},-\mathrm{Si}{ }^{t} \mathrm{Bu}\right), 0.86\left(9 \mathrm{H}, \mathrm{s},-\mathrm{Si}^{t} \mathrm{Bu}\right), 0.07(3 \mathrm{H}, \mathrm{s},-\mathrm{SiMe}), 0.07(3 \mathrm{H}, \mathrm{s},-\mathrm{SiMe}), 0.06(6 \mathrm{H}, \mathrm{s},-$ SiMe), 0.04 (3H, s, -SiMe), 0.03 (3H, s, -SiMe)

${ }^{13} \mathrm{C}$ NMR $\left(125 \mathrm{MHz}, \mathrm{CDCl}_{3}\right) \delta 171.2,170.6,132.1,118.3,80.2,67.2,67.2,66.9,66.7,66.6$, 65.1, 45.5, 44.0, 42.9, 37.2 (x2), 28.1 (x3), 25.9 (x3), 25.8 (x3), 25.8 (x3), 18.0, 18.0, 17.9, -4.4, $4.4,-4.6,-4.7,-4.8,-4.8$

HRMS (ES ${ }^{+}$) Cacld for $\mathrm{C}_{37} \mathrm{H}_{76} \mathrm{O}_{8} \mathrm{Si}_{3} \mathrm{Na}$ : 755.4746 Found: 755.4745

$[\alpha]_{D}^{27}=-2.6^{\circ}\left(c=1.5, \mathrm{CHCl}_{3}\right)$ 

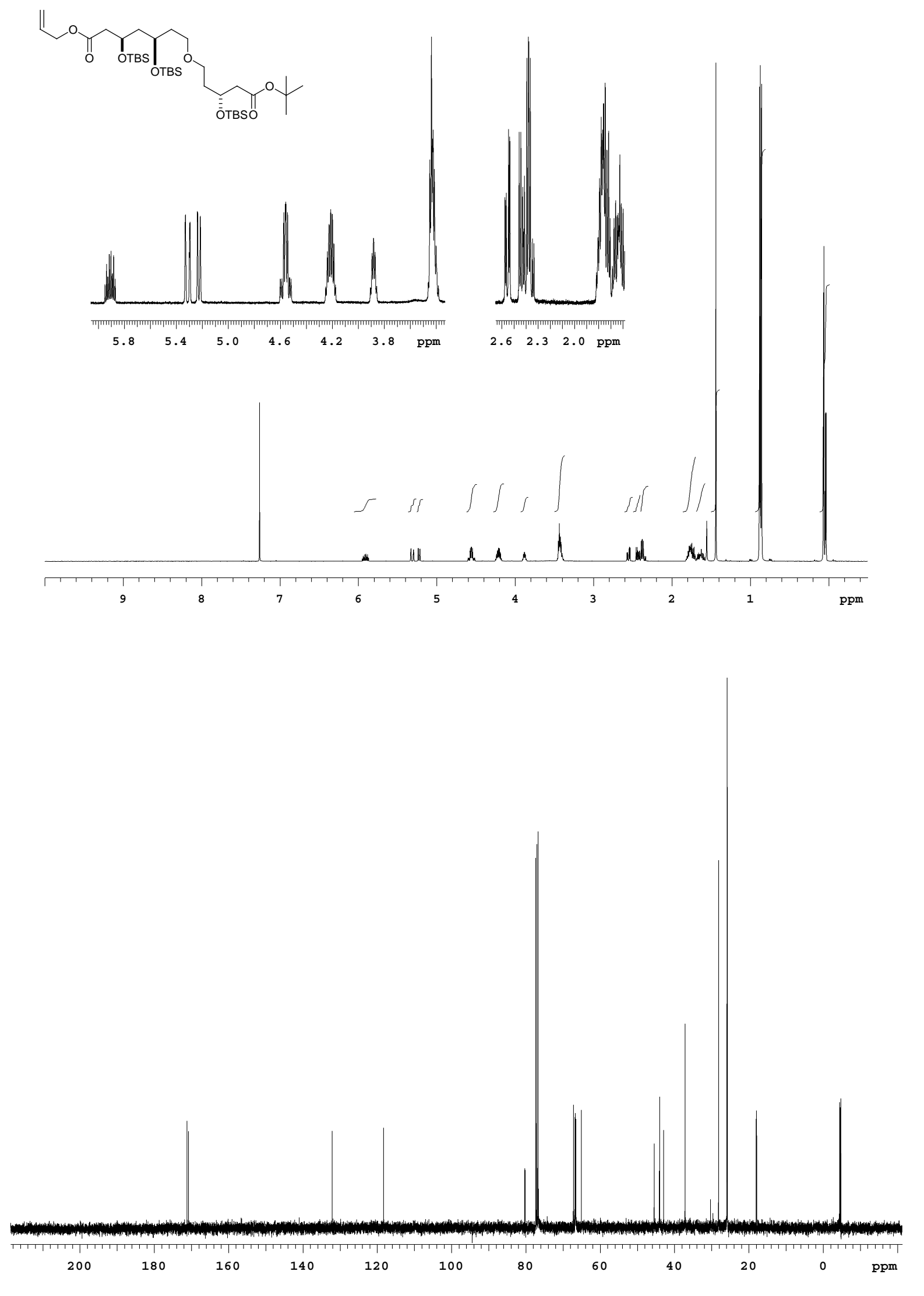


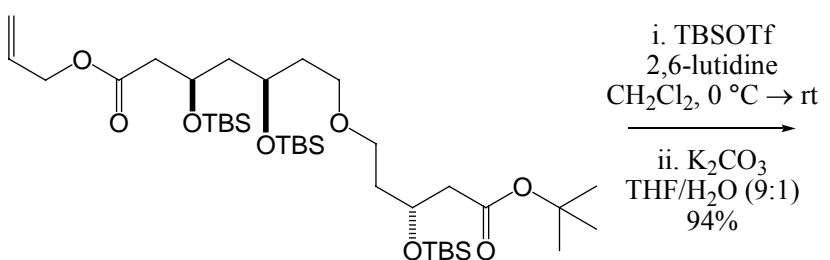

$11 b$

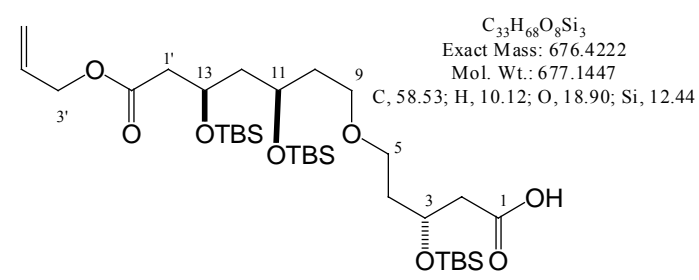

13

To a solution of $11 \mathrm{~b}(14.9 \mathrm{mg}, 0.020 \mathrm{mmol})$ in $\mathrm{CH}_{2} \mathrm{Cl}_{2}(40 \mu \mathrm{L})$ was added 2,6-Lutidine $(71 \mu \mathrm{L}, 0.610 \mathrm{mmol})$ and the reaction was cooled to $0{ }^{\circ} \mathrm{C}$. TBSOTf $(47 \mu \mathrm{L}, 0.205 \mathrm{mmol})$ was added and the reaction was allowed to stir at ambient temperature for $19 \mathrm{~h}$. The reaction was then diluted with $10 \% \mathrm{H}_{2} \mathrm{O}$ in THF $(200 \mu \mathrm{L})$ and $\mathrm{K}_{2} \mathrm{CO}_{3}(3.0 \mathrm{mg}, 0.022 \mathrm{mmol})$ was added and allowed to stir for $3 \mathrm{~h}$. The reaction was acidified to $\mathrm{pH} 4$ with $\mathrm{NaHSO}_{4} \cdot \mathrm{H}_{2} \mathrm{O}$ and stirred for 5 min. The solution was then diluted with $\mathrm{H}_{2} \mathrm{O}(500 \mu \mathrm{L})$ and extracted with $\mathrm{Et}_{2} \mathrm{O}(3 \times 1 \mathrm{~mL})$. The combined organic layers were dried over $\mathrm{MgSO}_{4}$, filtered, and reduced in vacuo. Flash chromatography $(10 \rightarrow 15 \%$ EtOAc : pentane $+0.1 \% \mathrm{AcOH})$ gave pure $13(12.7 \mathrm{mg}, 94 \%)$.

Data for 13:

$\mathbf{R}_{f}=0.31$ (20\% EtOAc, $80 \%$ pentane) - one black spot (p-anisaldehyde)

IR (film) 2955, 2929, 2886, 2857, 1738, 1713, 1472, 1463, 1410, 1386, 1361, 1257, 1165, 1105 , $1033,1005 \mathrm{~cm}^{-1}$

${ }^{1} \mathbf{H}$ NMR (400 MHz, $\left.\mathrm{CDCl}_{3}\right) \delta 5.91\left(1 \mathrm{H}, \mathrm{m}, \mathrm{C} 3{ }^{\prime}\right), 5.32\left(1 \mathrm{H}, \mathrm{m}, \mathrm{C} 5{ }^{\prime}(\mathrm{cis})\right), 5.23\left(1 \mathrm{H}, \mathrm{m}, \mathrm{C} 5{ }^{\prime}\right.$ (trans)), $4.56\left(2 \mathrm{H}, \mathrm{m}, \mathrm{C} 3{ }^{\prime}\right), 4.27(1 \mathrm{H}$, pent, $J=5.6 \mathrm{~Hz}, \mathrm{C} 3 / \mathrm{C} 13), 4.22(1 \mathrm{H}$, pent, $J=6.4 \mathrm{~Hz}$, C13/C3), $3.88(1 \mathrm{H}, \mathrm{m}, \mathrm{C} 11), 3.45(4 \mathrm{H}, \mathrm{m}, \mathrm{C} 5 \& \mathrm{C} 9), 2.62(1 \mathrm{H}, \mathrm{dd}, J=6.0,15.6 \mathrm{~Hz}, \mathrm{C} 2 / \mathrm{C} 1$ '), $2.55\left(1 \mathrm{H}, \mathrm{dd}, J=5.2,11.2 \mathrm{~Hz}, \mathrm{Cl}^{\prime} / \mathrm{C} 2\right), 2.51\left(1 \mathrm{H}, \mathrm{dd}, J=5.2,11.2 \mathrm{~Hz}, \mathrm{C} 1^{\prime} / \mathrm{C} 2\right), 2.45(1 \mathrm{H}, \mathrm{dd}, J$ $\left.=6.0,15.6 \mathrm{~Hz}, \mathrm{C} 2 / \mathrm{C} 1^{\prime}\right), 1.74(6 \mathrm{H}, \mathrm{m}, \mathrm{C} 4 \& \mathrm{C} 10 \& \mathrm{C} 12), 0.90\left(9 \mathrm{H}, \mathrm{s},-\mathrm{Si}^{t} \mathrm{Bu}\right), 0.88(9 \mathrm{H}, \mathrm{s},-$ $\left.\mathrm{Si}{ }^{t} \mathrm{Bu}\right), 0.86$ (9H, s, $\left.-\mathrm{Si}^{t} \mathrm{Bu}\right), 0.11$ (6H, s, -SiMe), 0.07 (3H, s, -SiMe), 0.06 (3H, s, -SiMe), 0.04 $(6 \mathrm{H}, \mathrm{s},-\mathrm{SiMe})$

${ }^{13}$ C NMR $\left(125 \mathrm{MHz}, \mathrm{CDCl}_{3}\right) \delta 171.3,132.1,118.4,67.4,66.8,66.7,66.7,66.5,65.2,45.4,42.9$, $41.9,37.0,36.8,25.9$ (x3), 25.8 (x3), 25.7 (x3), 18.0, 17.9, 17.9, -4.4, -4.5, -4.6, -4.7, -4.9, -4.9

HRMS (ES ${ }^{+}$) Cacld for $\mathrm{C}_{33} \mathrm{H}_{68} \mathrm{O}_{8} \mathrm{Si}_{3} \mathrm{Na}$ : 699.4120 Found: 699.4127

$[\alpha]_{D}^{27}=6.9^{\circ}\left(c=0.65, \mathrm{CHCl}_{3}\right)$ 

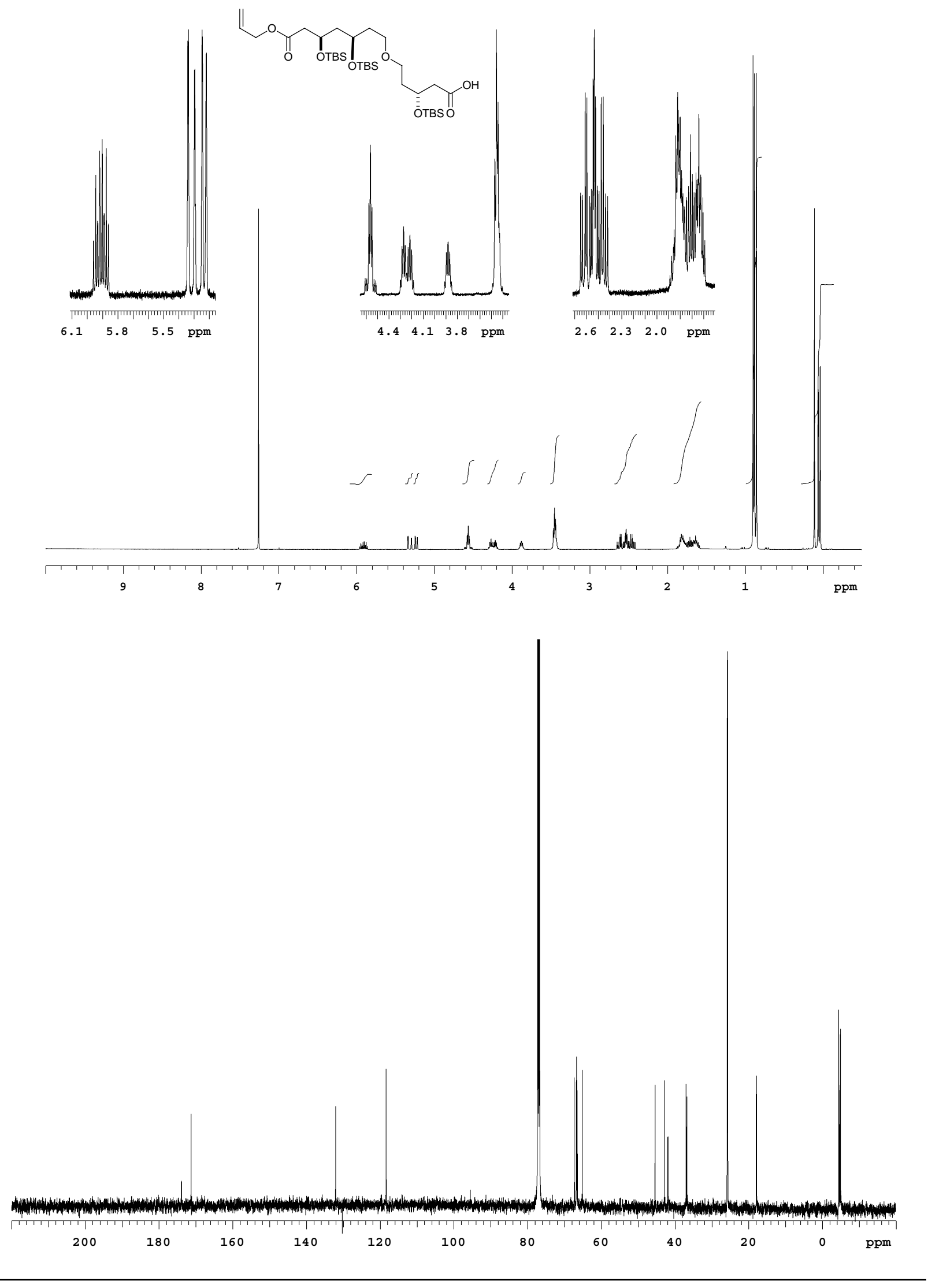


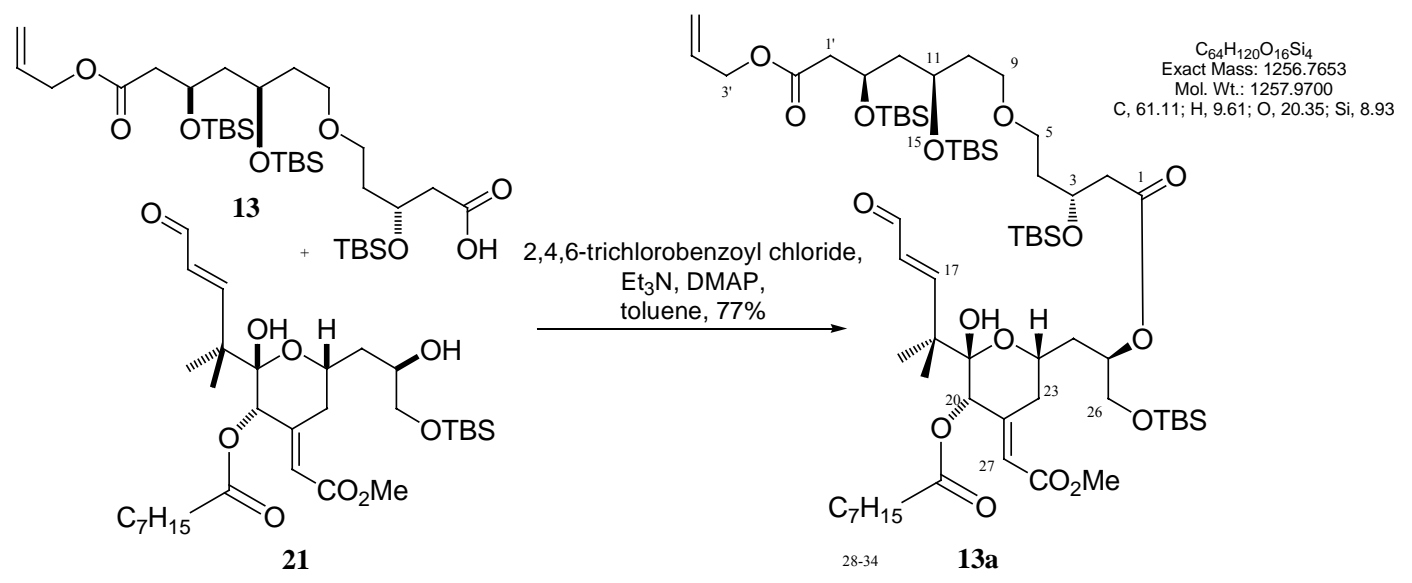

Acid 13 (18.5 mg, $0.027 \mathrm{mmol})$ was dissolved in toluene $(1.5 \mathrm{~mL}) . \mathrm{Et}_{3} \mathrm{~N}(15.4 \mu \mathrm{L}, 0.110$ mmol) was added, followed by 2,4,6-trichlorobenzoyl chloride $(4.6 \mu \mathrm{L}, 0.029 \mathrm{mmol})$. The reaction was allowed to stir for $5 \mathrm{~h}$ at rt. Alcohol 21 (15.8 $\mathrm{mg}, 0.026 \mathrm{mmol})$ and DMAP (6.5 mg, $0.053 \mathrm{mmol}$ ) were added as a solution in toluene $(1.5 \mathrm{~mL})$ and allowed to stir for $1.5 \mathrm{~h}$ at $\mathrm{rt}$, at which point direct flash chromatography $(10 \rightarrow 15 \%$ EtOAc : pentane $)$ gave 13a $(26.0 \mathrm{mg}, 77 \%)$ as a clear oil.

Data for 13a:

$\mathbf{R}_{f}=0.27$ (15\% EtOAc, $85 \%$ pentane) - one black, UV active spot (p-anisaldehyde)

IR (film) 3480, 2954, 2930, 2857, 1740, 1725, 1691, 1472, 1436, 1384, 1361, 1255, 1157, 1108, $1035 \mathrm{~cm}^{-1}$

${ }^{1} \mathbf{H}$ NMR $\left(500 \mathrm{MHz}, \mathrm{CDCl}_{3}\right) \delta 9.57(1 \mathrm{H}, \mathrm{d}, J=8.0 \mathrm{~Hz}, \mathrm{C} 15), 7.4(1 \mathrm{H}, \mathrm{d}, J=16.0 \mathrm{~Hz}, \mathrm{C} 17)$, 6.00, (1H, d, $J=1.4 \mathrm{~Hz}, \mathrm{C} 27), 5.96(1 \mathrm{H}, \mathrm{dd}, J=7.5,16.0 \mathrm{~Hz}, \mathrm{C} 16), 5.91$ (1H, ddt, $J=5.5,16.0$, $\left.5.5 \mathrm{~Hz}, \mathrm{C}^{\prime}\right), 5.31\left(1 \mathrm{H}, \mathrm{dd}, J=1.5,17.5 \mathrm{~Hz}, \mathrm{C}^{\prime}(\mathrm{cis})\right), 5.23\left(1 \mathrm{H}, \mathrm{dd}, J=1.2,10.5 \mathrm{~Hz}, \mathrm{C}^{\prime}\right.$ (trans)), 5.23 (1H, m, C25), $5.11(1 \mathrm{H}, \mathrm{s}, \mathrm{C} 20), 4.56\left(2 \mathrm{H}, \mathrm{m}, \mathrm{C} 3{ }^{\prime}\right), 4.21(2 \mathrm{H}, \mathrm{m}, \mathrm{C} 3$ \& C13), 3.85 $(2 \mathrm{H}, \mathrm{m}, \mathrm{C} 11$ \& C23), 3.69 (2H, m, C26), $3.68(3 \mathrm{H}, \mathrm{s},-\mathrm{OMe}), 3.66(1 \mathrm{H}, \mathrm{m}, \mathrm{C} 22), 3.42(4 \mathrm{H}, \mathrm{t}, J=$ $6.5 \mathrm{~Hz}, \mathrm{C} 5$ \& C9), $\left.3.18(1 \mathrm{H}, \mathrm{s}, \mathrm{OH}), 2.55(1 \mathrm{H}, \mathrm{dd}, J=5.0,15.0 \mathrm{~Hz}, \mathrm{C} 2 / \mathrm{C} 1)^{\prime}\right), 2.49(2 \mathrm{H}, \mathrm{d}, J=6.0$ $\left.\mathrm{Hz}, \mathrm{C} 1{ }^{\prime} / \mathrm{C} 2\right), 2.43$ (1H, dd, $J=7.0,15.0 \mathrm{~Hz}, \mathrm{C} 2 / \mathrm{C} 1$ '), 2.08 (1H, m, C28), 2.06 (1H, m, C22), 2.03 (1H, m, C28), $1.92(1 \mathrm{H}, \mathrm{m}, \mathrm{C} 4 / \mathrm{C} 10 / \mathrm{C} 12 / \mathrm{C} 24), 1.76(4 \mathrm{H}, \mathrm{m}, \mathrm{C} 4 / \mathrm{C} 10 / \mathrm{C} 12 / \mathrm{C} 24), 1.62$ (3H, m, $\mathrm{C} 4 / \mathrm{C} 10 / \mathrm{C} 12 / \mathrm{C} 24), 1.49$ (2H, m, C29), $1.22(8 \mathrm{H}, \mathrm{m}, \mathrm{C} 30-\mathrm{C} 33), 1.20\left(3 \mathrm{H}, \mathrm{s}, \mathrm{CH}_{3}\right), 1.15(3 \mathrm{H}, \mathrm{s}$, $\left.\mathrm{CH}_{3}\right), 0.91\left(9 \mathrm{H}, \mathrm{s}, \mathrm{SiC}\left(\mathrm{CH}_{3}\right)_{3}\right), 0.88\left(9 \mathrm{H}, \mathrm{s}, \mathrm{SiC}\left(\mathrm{CH}_{3}\right)_{3}\right), 0.86\left(9 \mathrm{H}, \mathrm{s}, \mathrm{SiC}\left(\mathrm{CH}_{3}\right)_{3}\right), 0.86(3 \mathrm{H}, \mathrm{m}$, C34), 0.84 (9H, s, SiC( $\left.\left.\mathrm{CH}_{3}\right)_{3}\right), 0.08$ (3H, s, SiMe), 0.07 (3H, s, SiMe), 0.07 (3H, s, SiMe), 0.06 (3H, s, SiMe), 0.05 (3H, s, SiMe), 0.04 (3H, s, SiMe), 0.03 (3H, s, SiMe), 0.02 (3H, s, SiMe)

${ }^{13}$ C NMR $\left(125 \mathrm{MHz}, \mathrm{CDCl}_{3}\right) \delta 194.9,172.7,172.0,171.4,166.7,166.7,150.7,132.4,127.7$, 121.0, 118.6, 99.8, 72.8, 71.7, 67.6, 67.3, 67.1, 66.8, 66.8, 66.4, 65.4, 65.2, 51.5, 46.0, 45.7, 43.2, 43.1, 37.7, 37.3, 37.3, 34.8, 31.9, 31.1, 29.2, 29.1, 26.1 (x3), 26.1 (x3), 26.1 (x3), 26.0 (x3), 24.7, $23.2,22.8,20.3,18.6,18.3,18.2,18.2,14.3,-4.2(\mathrm{x} 2),-4.3,-4.4,-4.5,-4.5,-5.0,-5.1$

HRMS $\left(\mathrm{ES}^{+}\right)$Cacld for $\mathrm{C}_{64} \mathrm{H}_{120} \mathrm{O}_{16} \mathrm{Si}_{3} \mathrm{Na}$ : 1279.7551 Found: 1279.7568 
$[\alpha]_{D}^{27}=-22.9^{\circ}\left(c=0.9, \mathrm{CHCl}_{3}\right)$ 

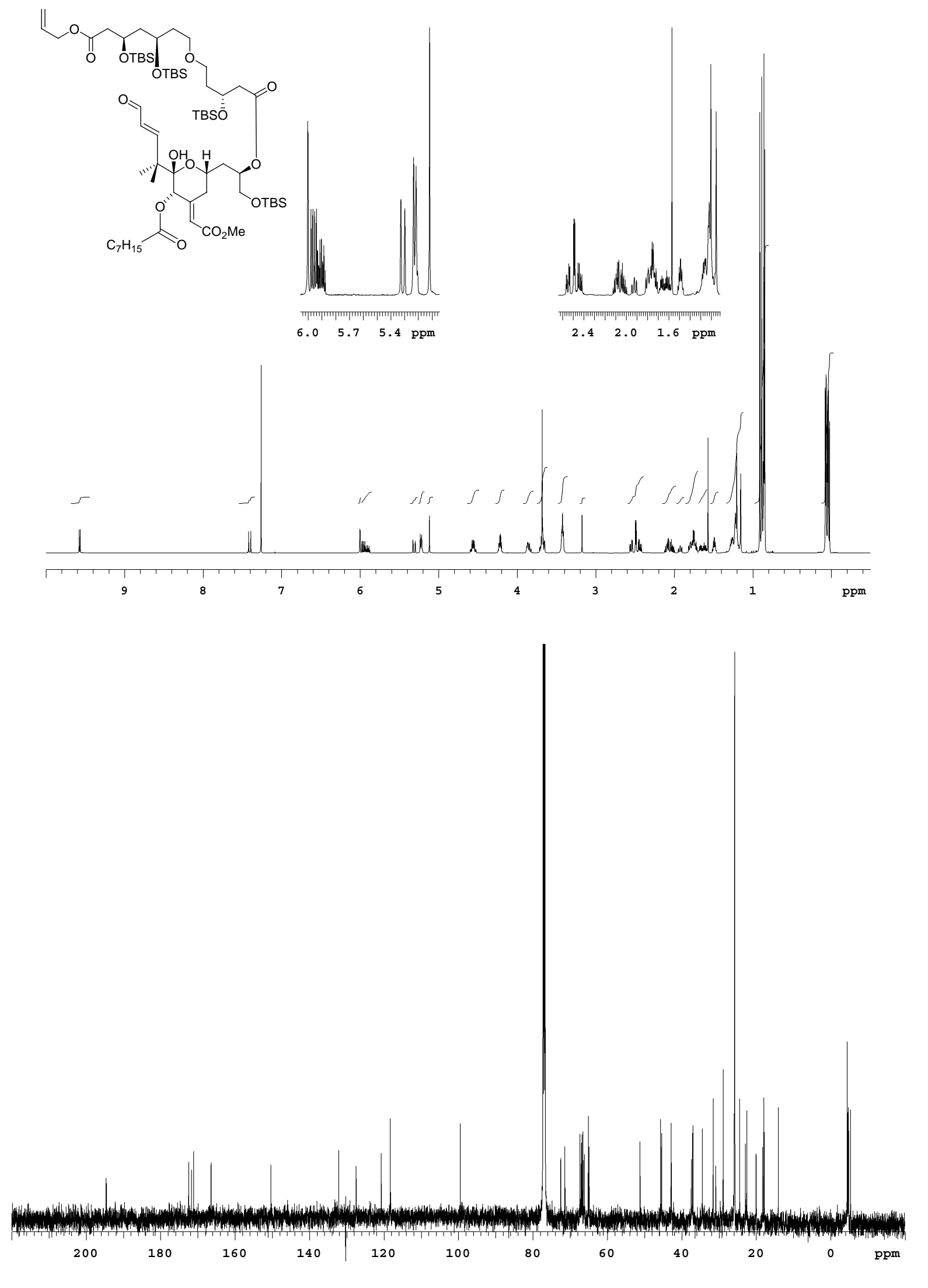

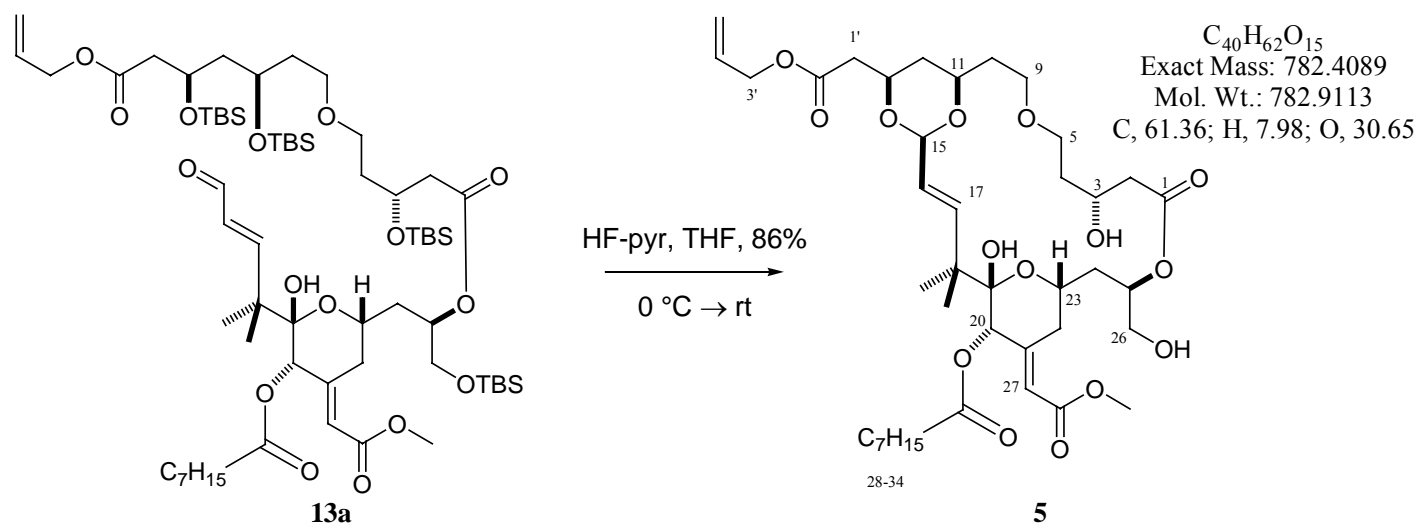

To a solution of 13a $(7.4 \mathrm{mg}, 0.006 \mathrm{mmol})$ in THF $(2 \mathrm{~mL})$ in a polyproplyene vialwas added HF-pyr $(0.4 \mathrm{~mL})$ was added at $0{ }^{\circ} \mathrm{C}$ and allowed to stir at $\mathrm{rt}$ for $4 \mathrm{~h}$. The reaction was quenched with satd. aq. $\mathrm{NaHCO}_{3}(20 \mathrm{~mL})$. The solution was diluted with $\mathrm{H}_{2} \mathrm{O}(5 \mathrm{~mL})$ and extracted with EtOAc $(4 \times 20 \mathrm{~mL})$. The combined organic layer was dried over $\mathrm{Na}_{2} \mathrm{SO}_{4}$, filtered and reduced in vacuo. Flash chromatography ( $60 \rightarrow 80 \%$ EtOAc : pentane) gave analog 5 as an amorphous solid (3.9 mg, 86\%).

Data for 5:

$\mathbf{R}_{f}=0.23$ ( $80 \%$ EtOAc, $20 \%$ pentane) - one black, UV active spot (p-anisaldehyde)

IR (film) 3460, 3338, 2925, 2858, 1734, 1669, 1436, 1384, 1363, 1347, 1283, 1260, 1230, 1160 , $1135,1121,1053 \mathrm{~cm}^{-1}$

${ }^{1} \mathbf{H}$ NMR $\left(500 \mathrm{MHz}, \mathrm{CDCl}_{3}\right) \delta 5.99(1 \mathrm{H}, \mathrm{d}, J=1.6 \mathrm{~Hz}, \mathrm{C} 27), 5.96(1 \mathrm{H}, \mathrm{d}, J=16.0 \mathrm{~Hz}, \mathrm{C} 17)$, $5.89\left(1 \mathrm{H}, \mathrm{ddt}, J=5.5,5.5,16.0 \mathrm{~Hz}, \mathrm{C} 4{ }^{\prime}\right), 5.39(1 \mathrm{H}, \mathrm{dd}, J=7.5,16.0 \mathrm{~Hz}, \mathrm{C} 16), 5.35(1 \mathrm{H}, \mathrm{m}$, C25), $5.31\left(1 \mathrm{H}, \mathrm{dd}, J=1.4,17.0 \mathrm{~Hz}, \mathrm{C} 5^{\prime}(\mathrm{cis})\right), 5.22\left(1 \mathrm{H}, \mathrm{dd}, J=1.2,10.5 \mathrm{~Hz}, \mathrm{C} 5^{\prime}\right.$ (trans)), 5.14 $(1 \mathrm{H}, \mathrm{s}, \mathrm{C} 20), 5.06(1 \mathrm{H}, \mathrm{d}, J=7.5 \mathrm{~Hz}, \mathrm{C} 15), 4.59(2 \mathrm{H}, \mathrm{d}, J=5.5 \mathrm{~Hz}, \mathrm{C} 3$ '), $4.24(1 \mathrm{H}, \mathrm{m}, \mathrm{C} 3), 4.19$ $(1 \mathrm{H}, \mathrm{m}, \mathrm{C} 13), 4.05(1 \mathrm{H}, \mathrm{m}, \mathrm{C} 23), 3.93(1 \mathrm{H}, \mathrm{m}, \mathrm{C} 11), 3.86(1 \mathrm{H}, \mathrm{dd}, J=3.0,12.0 \mathrm{~Hz}, \mathrm{C} 26), 3.71$ $(1 \mathrm{H}, \mathrm{m}, \mathrm{C} 22), 3.68(3 \mathrm{H}, \mathrm{s},-\mathrm{COOMe}), 3.66(1 \mathrm{H}, \mathrm{m}, \mathrm{C} 26), 3.64(1 \mathrm{H}, \mathrm{m}, \mathrm{C} 5), 3.63(1 \mathrm{H}, \mathrm{m}, \mathrm{C} 9)$, $3.58(1 \mathrm{H}, \mathrm{m}, \mathrm{C} 5), 3.40(1 \mathrm{H}, \mathrm{m}, \mathrm{C} 9), 2.70\left(1 \mathrm{H}, \mathrm{dd}, J=6.0,15.5 \mathrm{~Hz}, \mathrm{C} 1{ }^{\prime}\right), 2.52(2 \mathrm{H}, \mathrm{m}, \mathrm{C} 2), 2.46$ $(1 \mathrm{H}, \mathrm{dd}, J=7.5,15.5 \mathrm{~Hz}, \mathrm{C} 1$ '), $2.29(2 \mathrm{H}, \mathrm{m}, \mathrm{C} 28), 2.05(1 \mathrm{H}, \mathrm{m}, \mathrm{C} 22), 2.00(1 \mathrm{H}, \mathrm{m}, \mathrm{C} 24), 1.95$ $(1 \mathrm{H}, \mathrm{m}, \mathrm{C} 10), 1.80(1 \mathrm{H}, \mathrm{m}, \mathrm{C} 24), 1.63(1 \mathrm{H}, \mathrm{m}, \mathrm{C} 12), 1.61(2 \mathrm{H}, \mathrm{m}, \mathrm{C} 29), 1.58(1 \mathrm{H}, \mathrm{m}, \mathrm{C} 10), 1.55$ $(2 \mathrm{H}, \mathrm{m}, \mathrm{C} 4), 1.40(1 \mathrm{H}, \mathrm{dt}, J=11.5,12.5 \mathrm{~Hz}, \mathrm{C} 12), 1.27$ (8H, m (br), C30-C33), 1.17 (3H, s, $\left.\mathrm{C}\left(\mathrm{CH}_{3}\right)_{2}\right), 1.03\left(3 \mathrm{H}, \mathrm{s},-\mathrm{C}\left(\mathrm{CH}_{3}\right)_{2}\right), 0.87(3 \mathrm{H}, \mathrm{m}, \mathrm{C} 34)$

${ }^{13}$ C NMR $\left(125 \mathrm{MHz}, \mathrm{CDCl}_{3}\right) \delta 172.3,172.1,170.2,167.0,151.6,142.6,131.9,125.4,119.8$, $118.2,102.4,98.9,75.4,74.1,72.3,71.6,69.9,68.7,68.0,65.7,65.2,64.6,51.1,45.2,42.1,40.9$, $37.4,36.1$ (x2), 34.6, 33.1, 31.6, 31.0, 29.0, 28.9, 24.7, 24.3, 25.6, 19.4, 14.1

HRMS (ES ${ }^{+}$) Cacld for $\mathrm{C}_{40} \mathrm{H}_{62} \mathrm{O}_{15} \mathrm{Na}: 805.3986$ Found: 805.3981

$[\alpha]_{D}^{27}=-5.1^{\circ}\left(c=0.2, \mathrm{CHCl}_{3}\right)$ 

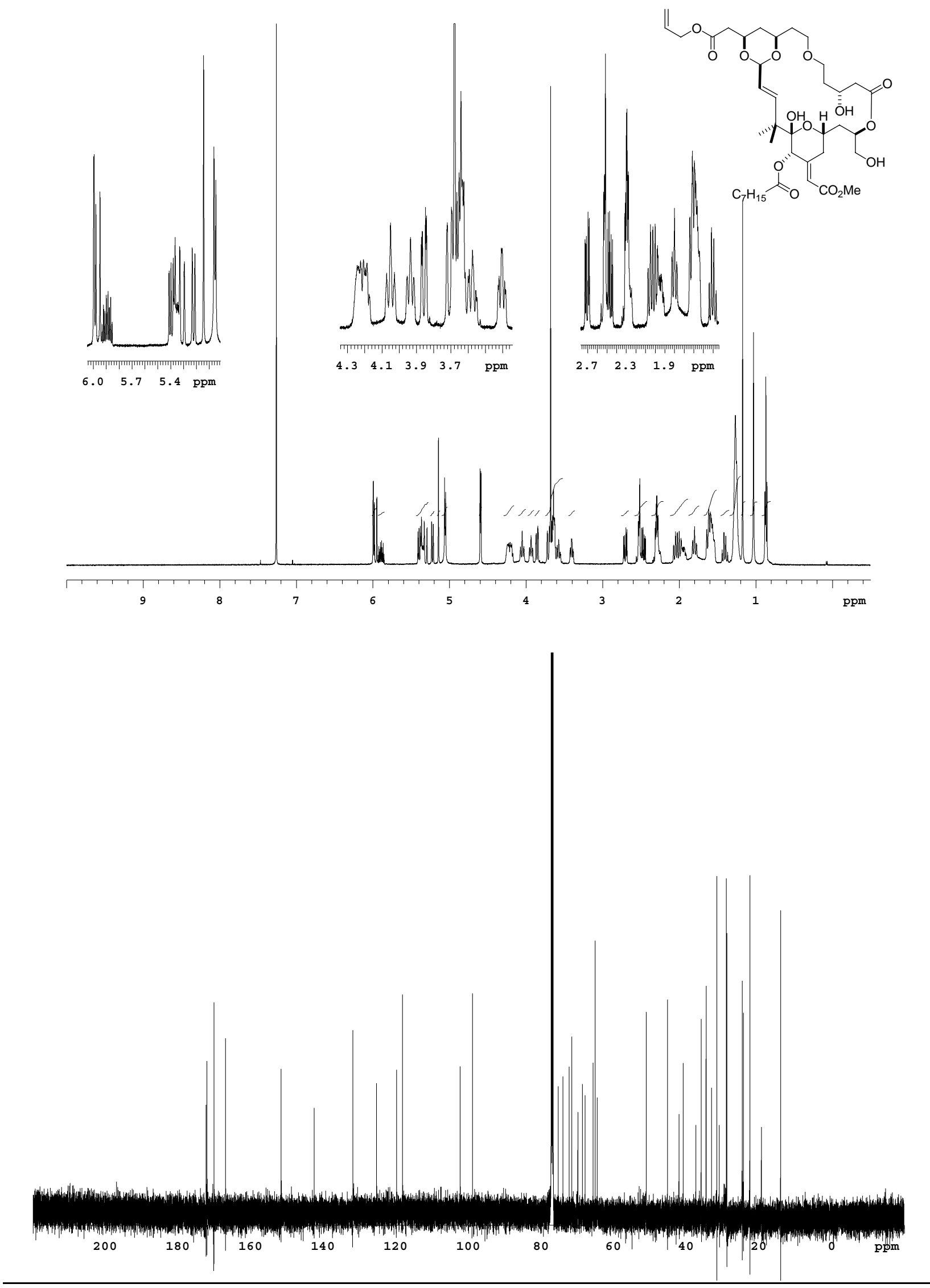


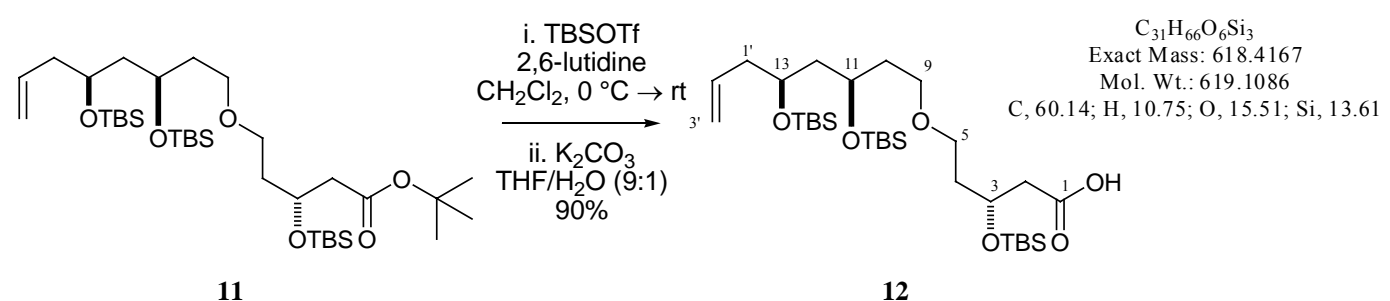

Ester 11 (12.7 mg, $0.019 \mathrm{mmol})$ was dissolved in $\mathrm{CH}_{2} \mathrm{Cl}_{2}(40 \mu \mathrm{L})$. 2,6-Lutidine ( $67 \mu \mathrm{L}$, $0.575 \mathrm{mmol})$ was added and the reaction cooled to $0{ }^{\circ} \mathrm{C}$. TBSOTf $(45 \mu \mathrm{L}, 0.196 \mathrm{mmol})$ was added and the reaction was allowed to stir at ambient temperature for $18 \mathrm{~h}$. The reaction was then diluted with $10 \% \mathrm{H}_{2} \mathrm{O}$ in THF $(200 \mu \mathrm{L})$ and $\mathrm{K}_{2} \mathrm{CO}_{3}(4.0 \mathrm{mg}, 0.029 \mathrm{mmol})$ was added and allowed to stir for $3 \mathrm{~h}$. The reaction was acidified to $\mathrm{pH} 4$ with $\mathrm{NaHSO}_{4} \cdot \mathrm{H}_{2} \mathrm{O}$ and stirred for 5 min. The solution was then diluted with $\mathrm{H}_{2} \mathrm{O}(500 \mu \mathrm{L})$ and extracted with $\mathrm{Et}_{2} \mathrm{O}(3 \times 1 \mathrm{~mL})$. The combined organic layers were dried over $\mathrm{Na}_{2} \mathrm{SO}_{4}$, filtered, and reduced in vacuo. Flash chromatography (15\% EtOAc : pentane $+0.5 \% \mathrm{AcOH})$ gave pure $12(10.6 \mathrm{mg}, 90 \%)$.

Data for 12:

$\mathbf{R}_{f}=0.43$ (20\% EtOAc, $80 \%$ pentane) - one black spot (p-anisaldehyde)

IR (film) 2955, 2929, 2886, 2858, 1713, 1642, 1472, 1463, 1386, 1361, 1257, 1112, $1005 \mathrm{~cm}^{-1}$

${ }^{1} \mathbf{H}$ NMR $\left(500 \mathrm{MHz}, \mathrm{CDCl}_{3}\right) \delta 10.00(1 \mathrm{H}, \mathrm{s}(\mathrm{br}),-\mathrm{COOH}), 5.79(1 \mathrm{H}, \mathrm{m}, \mathrm{C} 2 '), 5.03(2 \mathrm{H}, \mathrm{m}, \mathrm{C} 3 ')$, $4.27(1 \mathrm{H}, \mathrm{m}, \mathrm{C} 3), 3.89(1 \mathrm{H}, \mathrm{m}, \mathrm{C} 11), 3.78(1 \mathrm{H}, \mathrm{m}, \mathrm{C} 13), 3.45(4 \mathrm{H}, \mathrm{m}, \mathrm{C} 5$ \& C9), $2.62(1 \mathrm{H}, \mathrm{dd}, J$ $=5.5,15.5 \mathrm{~Hz}, \mathrm{C} 2), 2.52(1 \mathrm{H}, \mathrm{dd}, J=5.0,15.5 \mathrm{~Hz}, \mathrm{C} 2), 2.25\left(1 \mathrm{H}, \mathrm{m}, \mathrm{C} 1^{\prime}\right), 2.16\left(1 \mathrm{H}, \mathrm{m}, \mathrm{C} 1^{\prime}\right)$, $1.85(2 \mathrm{H}, \mathrm{m}, \mathrm{C} 4), 1.80(1 \mathrm{H}, \mathrm{m}, \mathrm{C} 10), 1.65(2 \mathrm{H}, \mathrm{m}, \mathrm{C} 12), 1.60(1 \mathrm{H}, \mathrm{m}, \mathrm{C} 10), 0.90\left(9 \mathrm{H}, \mathrm{s},-\mathrm{Si}{ }^{t} \mathrm{Bu}\right)$, $0.88\left(9 \mathrm{H}, \mathrm{s},-\mathrm{Si}{ }^{i} \mathrm{Bu}\right), 0.88\left(9 \mathrm{H}, \mathrm{s},-\mathrm{Si}{ }^{t} \mathrm{Bu}\right), 0.11(6 \mathrm{H}, \mathrm{s},-\mathrm{SiMe}), 0.05$ (3H, s, $\left.-\mathrm{SiMe}\right), 0.05$ (6H, s, $\mathrm{SiMe}), 0.04$ (3H, s, -SiMe)

${ }^{13}$ C NMR $\left(125 \mathrm{MHz}, \mathrm{CDCl}_{3}\right) \delta 173.9,134.8,117.0,69.0,67.6,66.8,66.7,66.6,44.8,42.1,41.9$, $37.0,36.8,25.9$ (x3), 25.9 (x3), 25.7 (x3), 18.0, 18.0, 17.9, -4.3, -4.4, -4.5, -4.6, -4.9, -4.9

HRMS (ES ${ }^{+}$) Cacld for $\mathrm{C}_{31} \mathrm{H}_{66} \mathrm{O}_{6} \mathrm{Si}_{3} \mathrm{Na}: 641.4065$ Found: 641.4071

$[\alpha]_{D}^{27}=16.4^{\circ}\left(c=1.1, \mathrm{CHCl}_{3}\right)$ 

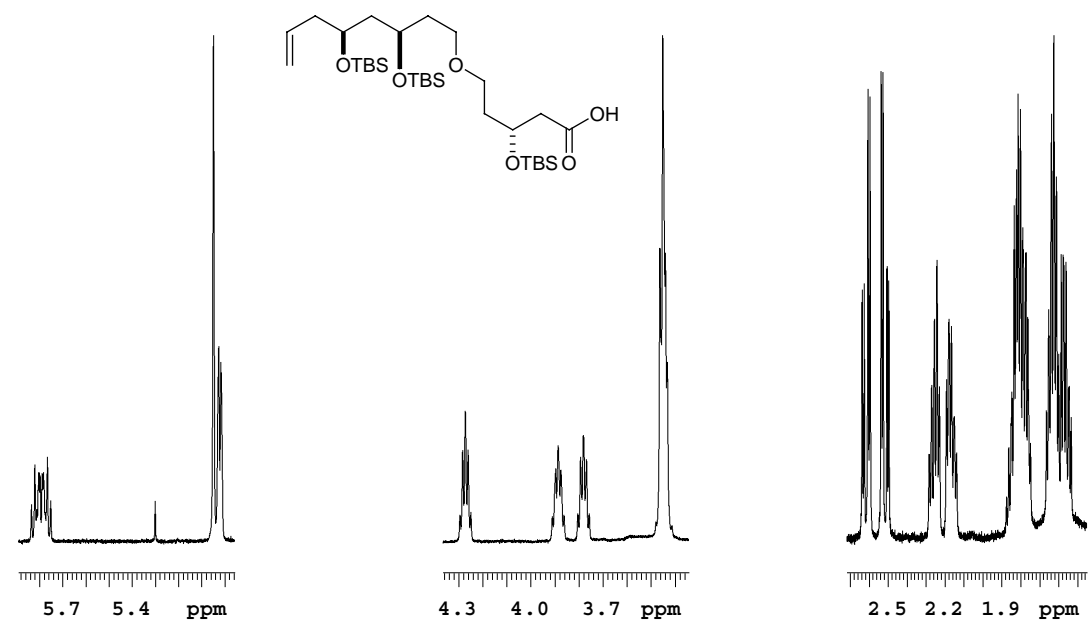

| $2.5 \quad 2.2 \quad 1.9 \mathrm{ppm}$
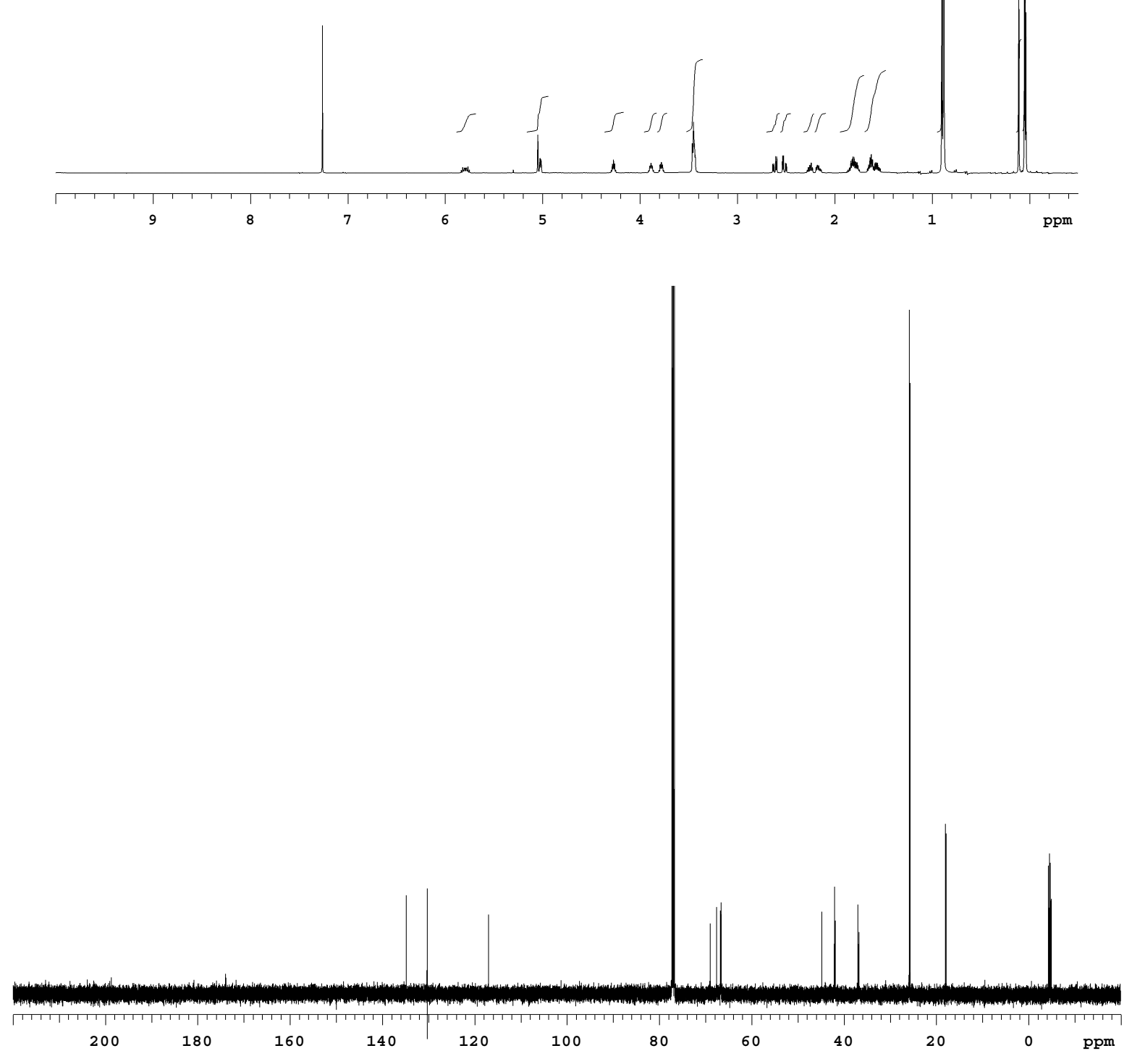

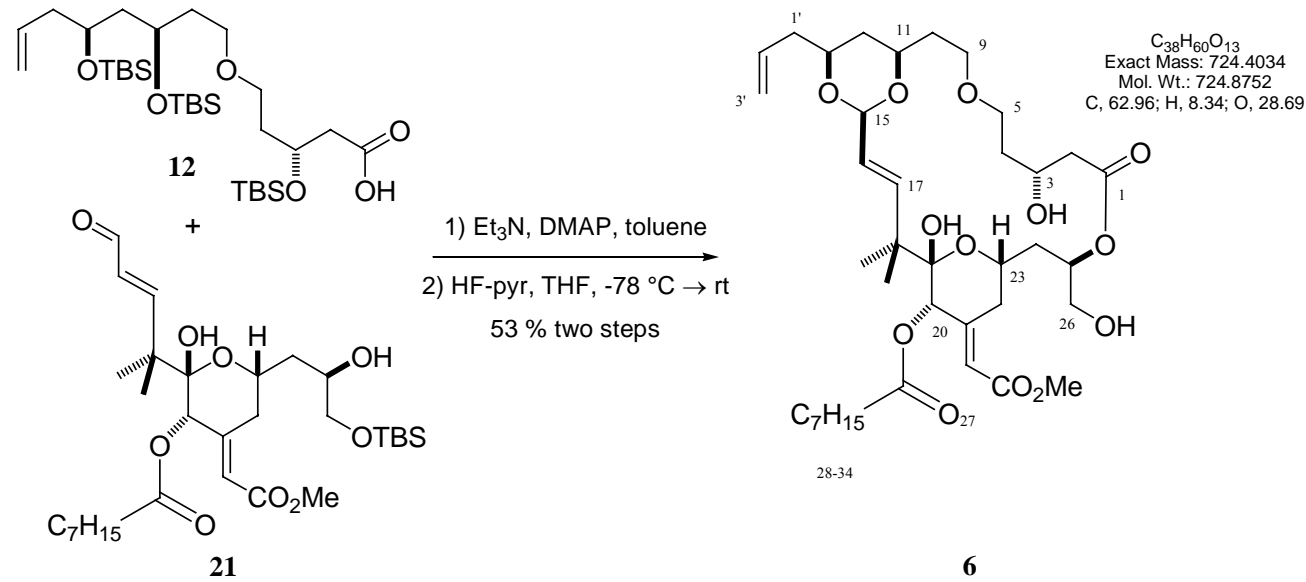

Acid $12(5.1 \mathrm{mg}, 0.008 \mathrm{mmol})$ was dissolved in toluene $(500 \mu \mathrm{L}) . \quad \mathrm{Et}_{3} \mathrm{~N}(4.5 \mu \mathrm{L}, 0.032$ mmol) was added, followed by 2,4,6-trichlorobenzoyl chloride $(1.4 \mu \mathrm{L}, 0.009 \mathrm{mmol})$. The reaction was allowed to stir for $5 \mathrm{~h}$ at $\mathrm{rt}$. Alcohol $21(5.2 \mathrm{mg}, 0.009 \mathrm{mmol})$ and DMAP (4.9 $\mathrm{mg}$, $0.040 \mathrm{mmol})$ were added as a solution in toluene $(500 \mu \mathrm{L})$ and allowed to stir for $1 \mathrm{~h} \mathrm{at} \mathrm{rt}$, at which point direct flash chromatography $(15 \%$ EtOAc : pentane) gave the product ester $(8.7 \mathrm{mg}$, $74 \%$ ) as a clear oil.

The product ester from the previous step $(6.9 \mathrm{mg}, 0.006 \mathrm{mmol})$ was dissolved in THF $(1.8 \mathrm{~mL})$ in a polypropylene vial. HF-pyr $(0.38 \mathrm{~mL})$ was added at $-78{ }^{\circ} \mathrm{C}$. The reaction was allowed to stir at $\mathrm{rt}$ for $6 \mathrm{~h}$, followed by quenching with satd. aq. $\mathrm{NaHCO}_{3}$. The solution was extracted with EtOAc $(4 \times 10 \mathrm{~mL})$ and the combined organic layer dried over $\mathrm{Na}_{2} \mathrm{SO}_{4}$, filtered, and reduced in vacuo. Flash chromatography $(60 \rightarrow 80 \%$ EtOAC : pentane) yielded analog 6 $(3.1 \mathrm{mg}, 73 \%)$ as an amorphous solid solid.

Data for 6:

$\mathbf{R}_{f}=0.32$ (80\% EtOAc, 20\% pentane) - one black, UV active spot (p-anisaldehyde)

IR (film) 3459, 3336, 2926, 2857, 1722, 1667, 1435, 1406, 1383, 1345, 1289, 1260, 1230, 1157 , $1135,1108,1060,1002 \mathrm{~cm}^{-1}$

${ }^{1}$ H NMR $\left(500 \mathrm{MHz}, \mathrm{CDCl}_{3}\right) \delta 6.00(1 \mathrm{H}, \mathrm{s}, \mathrm{C} 27), 5.97(1 \mathrm{H}, \mathrm{d}, J=16.0 \mathrm{~Hz}, \mathrm{C} 17), 5.79(1 \mathrm{H}$, dddd, $\left.J=7.5,7.5,9.5,17.5 \mathrm{~Hz}, \mathrm{C}^{\prime}\right), 5.42(1 \mathrm{H}, \mathrm{dd}, J=7.5,16.0 \mathrm{~Hz}, \mathrm{C} 16), 5.36(1 \mathrm{H}, \mathrm{m}, \mathrm{C} 25)$, $5.15(1 \mathrm{H}, \mathrm{s}, \mathrm{C} 20), 5.09(1 \mathrm{H}, \mathrm{m}, \mathrm{C} 2 '), 5.07(1 \mathrm{H}, \mathrm{s}, \mathrm{C} 19 \mathrm{OH}), 5.06\left(1 \mathrm{H}, \mathrm{m}, \mathrm{C} 2{ }^{\prime}\right), 5.02(1 \mathrm{H}, \mathrm{d}, J=$ $7.5 \mathrm{~Hz}, \mathrm{C} 15), 4.25$ (1H, m, C3), $4.06(2 \mathrm{H}, \mathrm{m}, \mathrm{C} 23$ \& C3OH), 3.87 (2H, m, C11 \& C26), 3.77 $(1 \mathrm{H}, \mathrm{m}, \mathrm{C} 13), 3.71(1 \mathrm{H}, \mathrm{m}, \mathrm{C} 22), 3.68\left(3 \mathrm{H}, \mathrm{s}, \mathrm{CO}_{2} \mathrm{Me}\right), 3.65(3 \mathrm{H}, \mathrm{m}, \mathrm{C} 5$ \& $\mathrm{C} 9$ \& $\mathrm{C} 26), 3.57$ $(1 \mathrm{H}, \mathrm{td}, J=2.8,12.5 \mathrm{~Hz}, \mathrm{C} 5), 3.39(1 \mathrm{H}, \mathrm{dt}, J=2.6,9.5 \mathrm{~Hz}, \mathrm{C} 9), 2.53(2 \mathrm{H}, \mathrm{m}, \mathrm{C} 2), 2.42(1 \mathrm{H}, \mathrm{m}$, C1'), 2.30 (2H, m, C28), $2.28(1 \mathrm{H}, \mathrm{m}, \mathrm{C} 4), 2.19\left(1 \mathrm{H}, \mathrm{m}, \mathrm{C} 1{ }^{\prime}\right), 2.06(1 \mathrm{H}, \mathrm{m}, \mathrm{C} 22), 2.00(1 \mathrm{H}, \mathrm{m}$, C24), $1.96(1 \mathrm{H}, \mathrm{m}, \mathrm{C} 10), 1.80(1 \mathrm{H}, \mathrm{m}, \mathrm{C} 24), 1.56(5 \mathrm{H}, \mathrm{m}, \mathrm{C} 4$ \& $\mathrm{C} 10$ \& $\mathrm{C} 12$ \& C29 (x2)), 1.34 (1H, m, C12), 1.27 (8H, m, C30-C33), 1.18 (3H, s, Me), $1.04(3 \mathrm{H}, \mathrm{s}, \mathrm{Me}), 0.87$ (3H, m, Me)

${ }^{13}$ C NMR $\left(125 \mathrm{MHz}, \mathrm{CDCl}_{3}\right) \delta 172.4,172.1,167.0,151.7,142.4,133.7,125.6,119.8,117.4$, $102.4,98.9,75.5,75.5,74.1,71.7,69.8,68.7,67.9,65.8,64.5,51.1,45.2,42.2,40.3,37.2,36.0$, $36.0,34.6,33.1,31.6,31.0,29.0,28.9,24.7,24.3,22.6,19.3,14.1$ 
HRMS (ES ${ }^{+}$) Cacld for $\mathrm{C}_{38} \mathrm{H}_{60} \mathrm{O}_{13} \mathrm{Na}$ : 747.3932 Found: 747.3942

$[\alpha]_{D}^{27}=-16.7^{\circ}\left(c=0.4, \mathrm{CHCl}_{3}\right)$ 

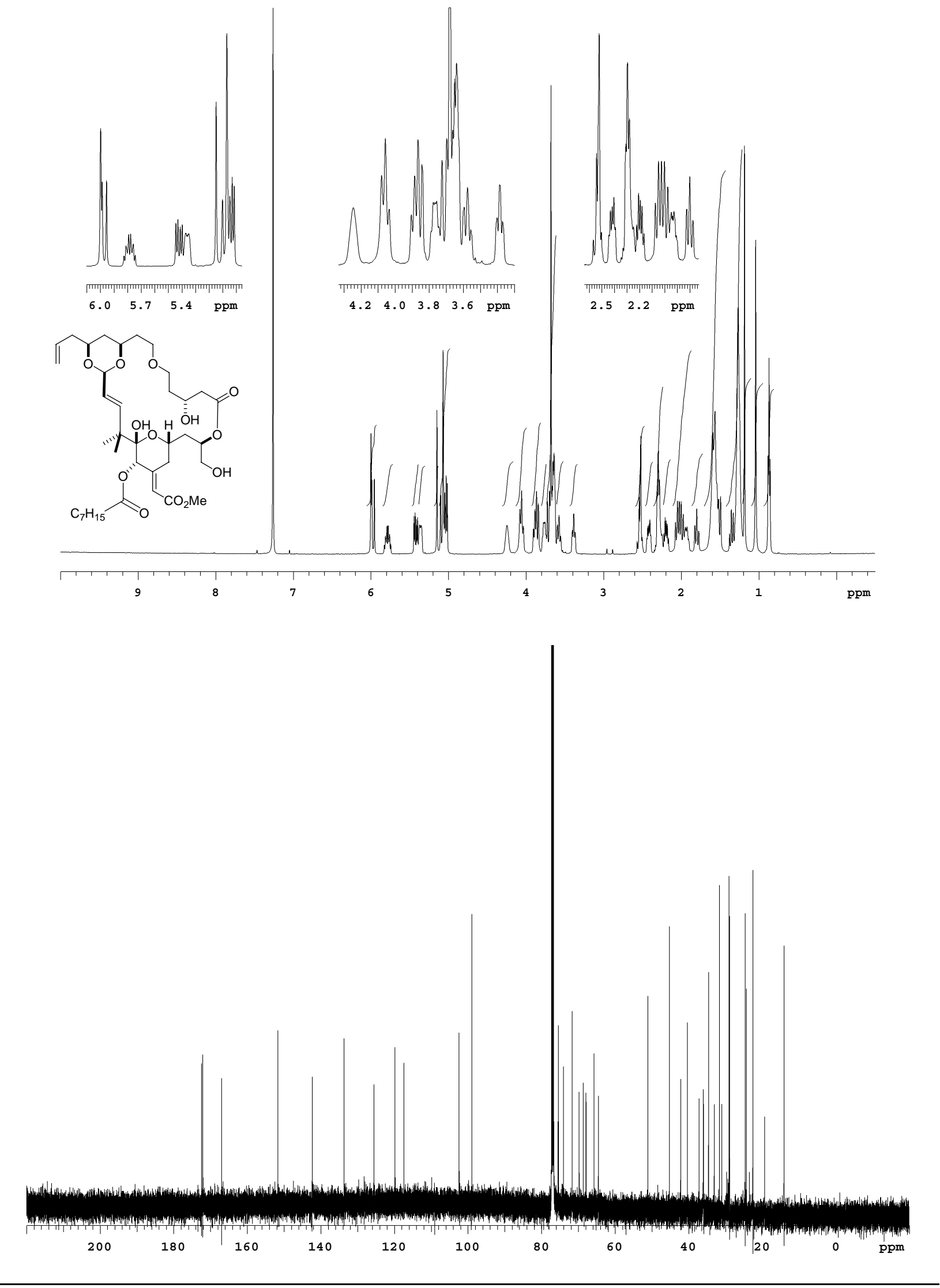


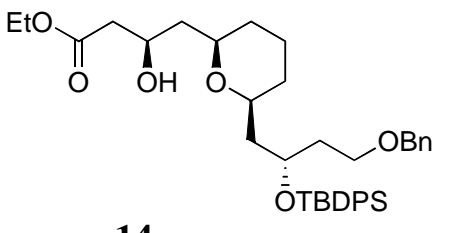

14

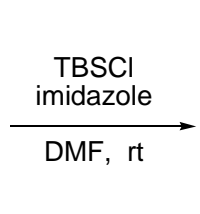

$\underset{\text { DMF, rt }}{\stackrel{\substack{\text { TBSCl } \\ \text { imidazole }}}{\longrightarrow}}$

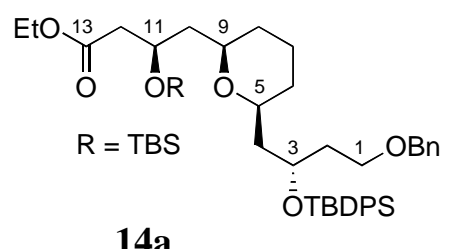

$14 a$

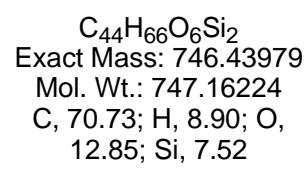

Exact Mass: 746.43979
Mol. Wt.: 747.16224
C, 70.73; $\mathrm{H}, 8.90 ; \mathrm{O}$,
12.85; $\mathrm{Si}, 7.52$

To a solution of alcohol $14(269 \mathrm{mg}, 0.425 \mathrm{mmol})^{\mathrm{i}}$ in DMF $(1.0 \mathrm{~mL})$, under $\mathrm{N}_{2}$ at $\mathrm{rt}$, was added a single portion of solid imidazole $(87 \mathrm{mg}, 1.28 \mathrm{mmol})$ followed by a single portion of solid TBSCl (128 mg, $0.849 \mathrm{mmol})$. The reaction was allowed to stir for $15 \mathrm{~h}$ and was then diluted with sat. aq. $\mathrm{NaHCO}_{3}(5 \mathrm{~mL})$ and extracted with $\mathrm{Et}_{2} \mathrm{O}(3 \times 5 \mathrm{~mL})$. The combined organic layers were dried over anhydrous $\mathrm{MgSO}_{4}$, filtered and concentrated in vacuo. Chromatography on silica (10\% EtOAc / pentane) gave $304 \mathrm{mg}$ of the silyl ether 14a as a colorless oil (96\%).

Data for 14a:

$\mathbf{R}_{f}=0.77$ (20\% EtOAc / petroleum ether)—one red/black spot w/ p-anisaldehyde stain.

IR (thin film): 3070, 2930, 2857, 1738, 1589, 1472, 1428, 1373, 1308, 1256, 1223, 1177, 1111 , $1006,966,939,901,836,777,736,702 \mathrm{~cm}^{-1}$.

${ }^{1} \mathbf{H}$ NMR (600 MHz, $\left.\mathrm{CDCl}_{3}\right): \delta 7.66(4 \mathrm{H}, \mathrm{m}, \mathrm{ArH}), 7.40(2 \mathrm{H}, \mathrm{m}, \mathrm{ArH}), 7.35$ (4H, m, ArH), 7.30 $(2 \mathrm{H}, \mathrm{m}, \mathrm{ArH}), 7.25$ (3H, m, ArH), 4.33 (2H, s, $\left.-\mathrm{CH}_{2} \mathrm{Ph}\right), 4.22(1 \mathrm{H}, \mathrm{m}, \mathrm{C} 11), 4.08$ (3H, m, C3 \& $\left.\mathrm{CO}_{2} \mathrm{CH}_{2} \mathrm{CH}_{3}\right), 3.46(2 \mathrm{H}, \mathrm{ddt}, J=9.4,17.8,6.8 \mathrm{~Hz}, \mathrm{C} 1), 3.23(2 \mathrm{H}, \mathrm{m}, \mathrm{C} 5 \& \mathrm{C} 9), 2.40$ (1H, dd, $J$ $=5.5,14.8 \mathrm{~Hz}, \mathrm{C} 12), 2.37(1 \mathrm{H}, \mathrm{dd}, J=6.8,14.8 \mathrm{~Hz}, \mathrm{C} 12), 1.77(2 \mathrm{H}, \mathrm{m}, \mathrm{C} 2), 1.69$ (2H, m, C7 \& C10), 1.61 (1H, ddd, $J=5.1,8.1,14.0 \mathrm{~Hz}, \mathrm{C} 4), 1.51(3 \mathrm{H}, \mathrm{m}, \mathrm{C} 4, \mathrm{C} 8$ \& C10), 1.29 (2H, m, C6 \& C7), $1.23\left(3 \mathrm{H}, \mathrm{t}, J=7.1,-\mathrm{CO}_{2} \mathrm{CH}_{2} \mathrm{CH}_{3}\right), 1.07(1 \mathrm{H}, \mathrm{ddt}, J=4.1,11.2,13.1 \mathrm{~Hz}, \mathrm{C} 6$ or C8), 1.03 $(9 \mathrm{H}, \mathrm{s}, t-\mathrm{Bu}), 0.97(1 \mathrm{H}, \mathrm{ddt}, J=4.1,11.0,13.1 \mathrm{~Hz}, \mathrm{C} 6$ or $\mathrm{C} 8), 0.84(9 \mathrm{H}, \mathrm{s}, t-\mathrm{Bu}), 0.022(3 \mathrm{H}, \mathrm{s},-$ OTBDMS), 0.014 (3H, s, -OTBDMS).

${ }^{13}$ C NMR (125 MHz, $\mathrm{CDCl}_{3}$ ): $\delta$ 171.7, 138.6, 135.89 (2C), 135.87 (2C), 134.5, 129.5, 129.4, 128.2 (2C), 127.55 (2C), 127.50 (2C), 127.46 (2C), 127.3 (2C), 74.5, 74.0, 72.7, 68.7, 66.9, 66.6, 60.2, 44.7, 44.0, 42.7, 37.6, 31.7, 31.3, 27.0 (3C), 25.8 (3C), 23.5, 19.5, 17.9, 14.2, -4.5, -4.8.

HRMS (MALDI): Calculated for $\mathrm{C}_{44} \mathrm{H}_{66} \mathrm{O}_{6} \mathrm{NaSi}_{2}$ [M+Na]: 769.4290. Found: 769.4311 .

Elemental Analysis: Calculated for $\mathrm{C}_{44} \mathrm{H}_{66} \mathrm{O}_{6} \mathrm{Si}_{2}$ : C 70.73; H 8.90. Found: $\mathrm{C} 70.53 ; \mathrm{H}$ 8.73. $[\alpha]_{D}^{23}=+7.1^{\circ}\left(c=1.84, \mathrm{CHCl}_{3}\right)$. 

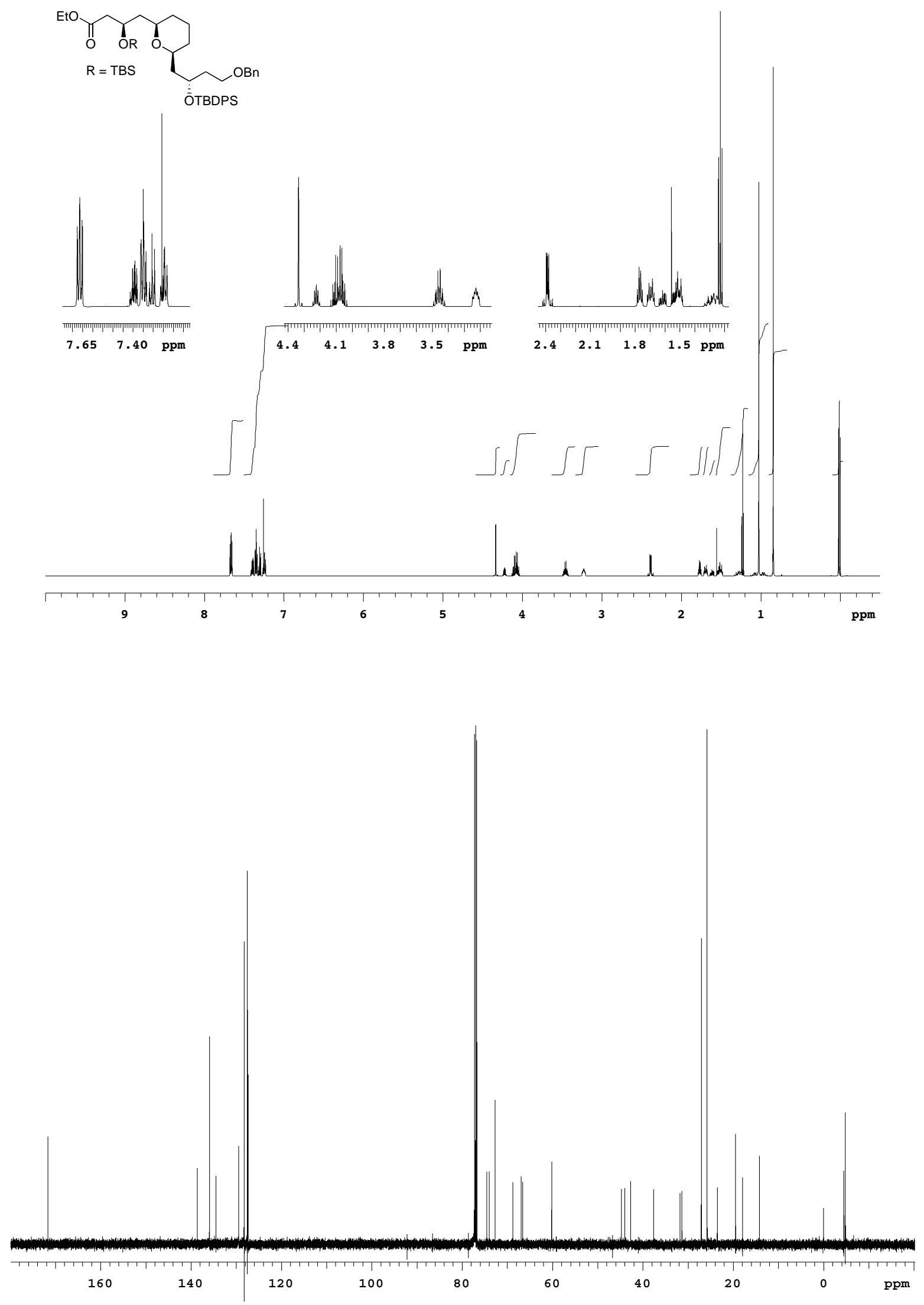

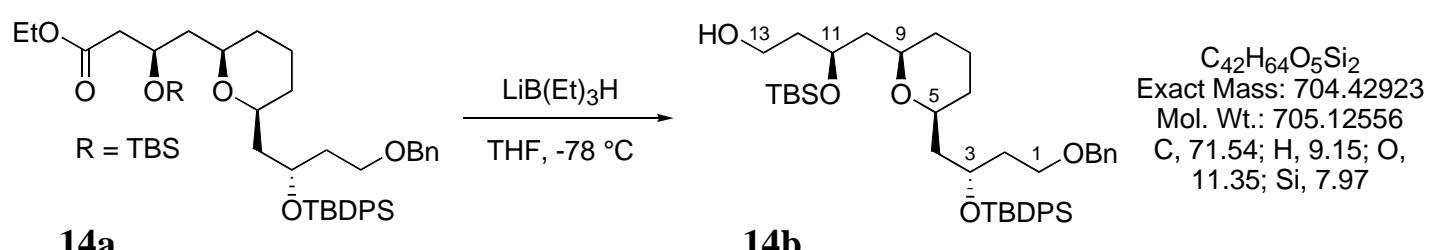

To a solution of ester 14a (191 mg, $0.256 \mathrm{mmol})$ in THF $(4.5 \mathrm{~mL})$, under $\mathrm{N}_{2}$ at $-78{ }^{\circ} \mathrm{C}$, was added Super Hydride $(1.0 \mathrm{~mL}$ of $1.0 \mathrm{M}$ solution in THF, $1.0 \mathrm{mmol}$ ) dropwise over $2 \mathrm{~min}$ via syringe. The reaction was allowed to stir for $15 \mathrm{~min}$ and then $\mathrm{H}_{2} \mathrm{O}_{2}(0.4 \mathrm{~mL}$ of a $30 \%$ aq. solution) followed by $\mathrm{NaOH}\left(0.1 \mathrm{~mL}\right.$ of a $1.0 \mathrm{M}$ aq. solution) were added via syringe at $0{ }^{\circ} \mathrm{C}$. The reaction was stirred at $0{ }^{\circ} \mathrm{C}$ for $5 \mathrm{~min}$ and then allowed to stir at $\mathrm{rt}$ for $15 \mathrm{~min}$. The reaction mixture was diluted with water $(3 \mathrm{~mL})$ and sat. aq. $\mathrm{Na}_{2} \mathrm{~S}_{2} \mathrm{O}_{3}(1 \mathrm{~mL})$ and then extracted with $\mathrm{Et}_{2} \mathrm{O}$ $(4 \times 6 \mathrm{~mL})$. The combined organic layers were dried over anhydrous $\mathrm{MgSO}_{4}$, filtered and concentrated in vacuo. Chromatography on silica (15\% EtOAc / pentane) gave $159 \mathrm{mg}$ of the alcohol $\mathbf{1 4 b}$ as a colorless oil (88\%).

Data for 14b:

$\mathbf{R}_{f}=0.38$ (20\% EtOAc / hexanes ether) - one black spot $\mathrm{w} / \mathrm{p}$-anisaldehyde stain.

IR (thin film): 2459, 3070, 2930, 2857, 1589, 1454, 1472, 1463, 1360, 1256, 1198, 1111, 1072, $938,836,775,736,702 \mathrm{~cm}^{-1}$.

${ }^{1} \mathbf{H}$ NMR $\left(600 \mathrm{MHz}, \mathrm{CDCl}_{3}\right): \delta 7.66(4 \mathrm{H}, \mathrm{m}, \mathrm{ArH}), 7.41(2 \mathrm{H}, \mathrm{m}, \mathrm{ArH}), 7.33(6 \mathrm{H}, \mathrm{m}, \mathrm{ArH}), 7.25$ $(3 \mathrm{H}, \mathrm{m}, \mathrm{ArH}), 4.35\left(1 \mathrm{H}, \mathrm{d}, J=11.9 \mathrm{~Hz},-\mathrm{CH}_{2} \mathrm{Ph}\right), 4.33\left(1 \mathrm{H}, \mathrm{d}, J=11.9 \mathrm{~Hz},-\mathbf{C H}_{2} \mathrm{Ph}\right), 4.04(2 \mathrm{H}$, m, C3 \& C11), 3.70 (1H, dddd, $J=4.6,4.6,8.9,11.0 \mathrm{~Hz}, \mathrm{C} 13), 3.59(1 \mathrm{H}$, ddt, $J=4.9,11.0,5.7$ $\mathrm{Hz}, \mathrm{C} 13), 3.45(2 \mathrm{H}, \mathrm{m}, \mathrm{C} 1), 3.16$ (1H, ddt, $J=8.6,11.0,2.8 \mathrm{~Hz}, \mathrm{C} 5$ or C9), 3.12 (1H, dddd, $J=$ $2.1,3.8,8.8,10.8 \mathrm{~Hz}, \mathrm{C} 5$ or C9), $2.62(1 \mathrm{H}, \mathrm{t}, J=5.6, \mathrm{C} 13-\mathrm{OH}), 1.65-1.83(5 \mathrm{H}, \mathrm{m}, \mathrm{C} 2, \mathrm{C} 7, \mathrm{C} 10$ \& C12), $1.60(1 \mathrm{H}, \mathrm{ddd}, J=5.4,8.5,14.1 \mathrm{~Hz}, \mathrm{C} 4), 1.51(3 \mathrm{H}, \mathrm{m}, \mathrm{C} 4, \mathrm{C} 10 \& \mathrm{C} 12), 1.43(1 \mathrm{H}, \mathrm{brd}$, $J=11.8 \mathrm{~Hz}, \mathrm{C} 8), 1.30(1 \mathrm{H}, \mathrm{qt}, J=13.3,4.1 \mathrm{~Hz}, \mathrm{C} 7), 1.23(1 \mathrm{H}, \mathrm{brd}, J=13.1 \mathrm{~Hz}, \mathrm{C} 6), 1.08(1 \mathrm{H}$, ddt, $J=4.0,11.2,13.0 \mathrm{~Hz}, \mathrm{C} 8), 1.02(9 \mathrm{H}, \mathrm{s}, t-\mathrm{Bu}), 0.99(1 \mathrm{H}, \mathrm{ddt}, J=4.2,11.5,13.0 \mathrm{~Hz}, \mathrm{C} 6)$, 0.87 (9H, s, t-Bu), 0.05 (3H, s, -OTBDMS), 0.03 (3H, s, -OTBDMS).

${ }^{13} \mathrm{C}$ NMR (125 MHz, $\mathrm{CDCl}_{3}$ ): $\delta$ 138.5, 135.9 (2C), 135.8 (2C), 134.6, 134.4, 129.51, 129.47, 128.2 (2C), 127.6 (2C), 127.5 (2C), 127.44 (2C), 127.40, 74.7, 74.1, 72.8, 69.2, 68.9, 67.0, 60.1, 44.8, 43.5, 37.8, 37.6, 31.84, 31.81, 27.0 (3C), 25.8 (3C), 23.6, 19.4, 17.9, -4.5, -4.8.

HRMS (MALDI): Calculated for $\mathrm{C}_{42} \mathrm{H}_{64} \mathrm{O}_{5} \mathrm{NaSi}_{2}[\mathrm{M}+\mathrm{Na}]$ : 727.4185. Found: 727.4165.

Elemental Analysis: Calculated for $\mathrm{C}_{42} \mathrm{H}_{64} \mathrm{O}_{5} \mathrm{Si}_{2}$ : C 71.54; H 9.15. Found: $\mathrm{C} 71.28 ; \mathrm{H} 9.35$.

$[\alpha]_{D}^{23}=+5.1^{\circ}\left(c=1.93, \mathrm{CHCl}_{3}\right)$. 

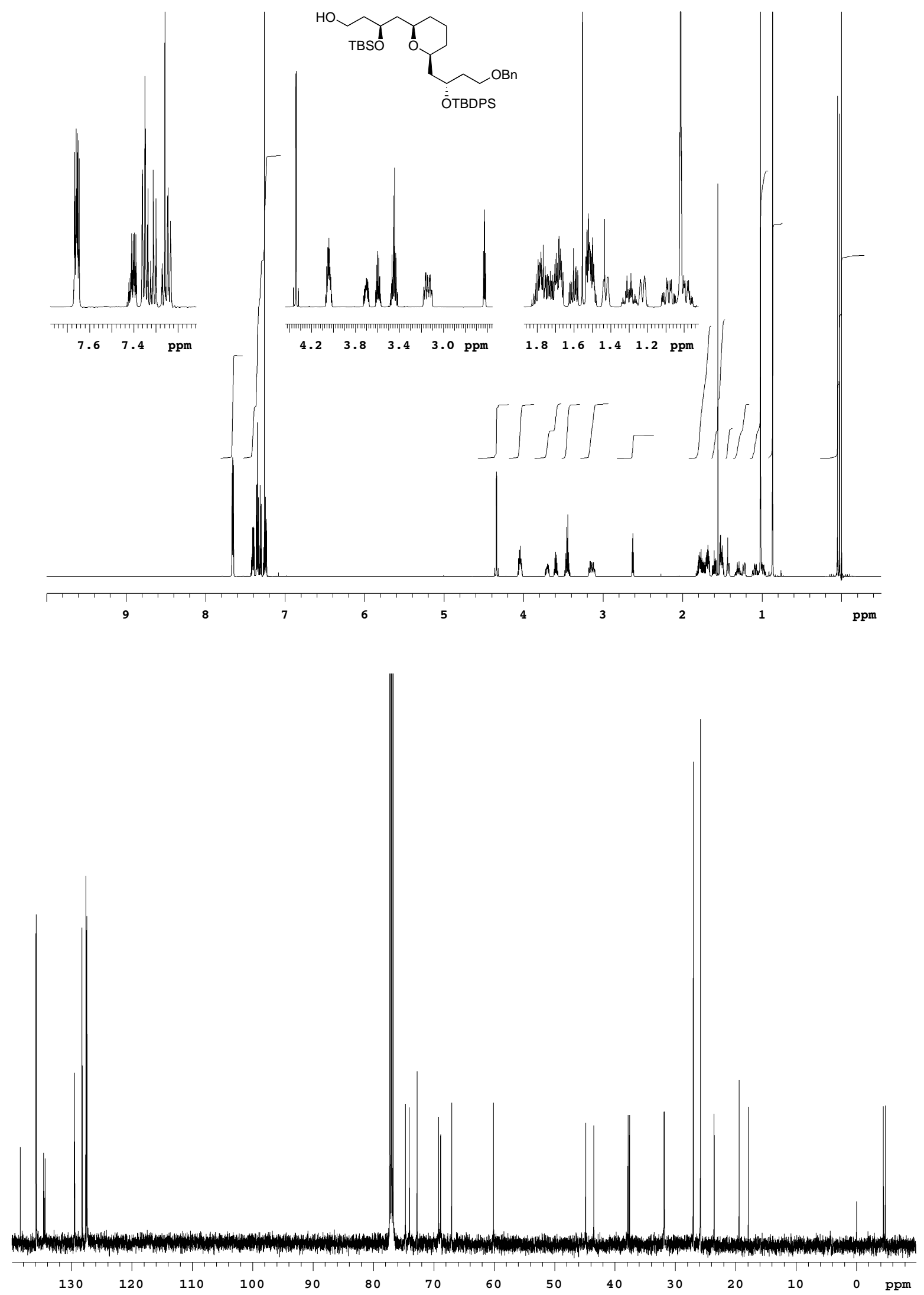

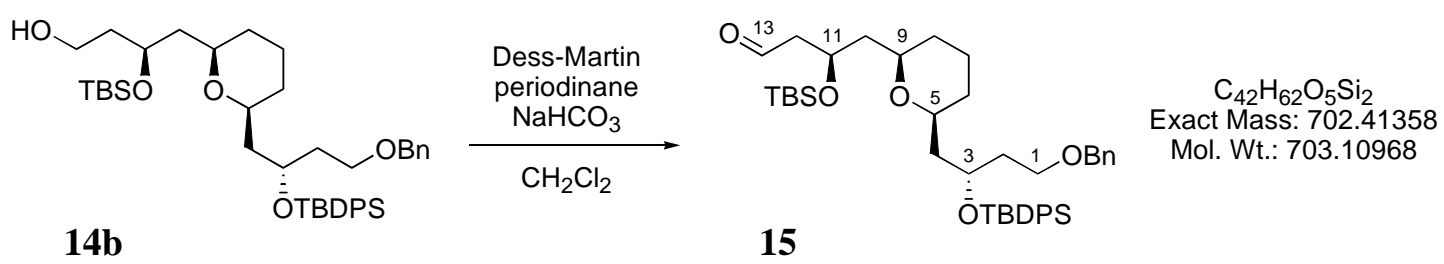

To a solution of alcohol $14 \mathbf{b}(159 \mathrm{mg}, 0.225 \mathrm{mmol})$ in THF $(4.5 \mathrm{~mL})$, under $\mathrm{N}_{2}$ at $0{ }^{\circ} \mathrm{C}$, was added a single portion of solid $\mathrm{NaHCO}_{3}(71 \mathrm{mg}, 0.84 \mathrm{mmol})$ followed by a single portion of Dess-Martin periodinane $(143 \mathrm{mg}, 0.337 \mathrm{mmol})$. The reaction was stirred at $0{ }^{\circ} \mathrm{C}$ for 5 min and then allowed to stir at $\mathrm{rt}$ for $1.5 \mathrm{~h}$. The reaction mixture was diluted with water $(4 \mathrm{~mL})$ and sat. aq. $\mathrm{Na}_{2} \mathrm{~S}_{2} \mathrm{O}_{3}(2 \mathrm{~mL})$ and extracted with $\mathrm{Et}_{2} \mathrm{O}(4 \times 6 \mathrm{~mL})$. The combined organic layers were dried over anhydrous $\mathrm{Na}_{2} \mathrm{SO}_{4}$, filtered and concentrated in vacuo. Chromatography on silica (15\% EtOAc / pentane) gave $143 \mathrm{mg}$ of the aldehyde 15 as a colorless oil (90\%).

Data for 15:

$\mathbf{R}_{f}=0.38$ (10\% EtOAc / hexanes ether) — one black spot w/ p-anisaldehyde stain.

IR (thin film): 3069, 2930, 2856, 2720, 1725, 1589, 1473, 1427, 1361, 1253, 1199, 1111, 1006, 938, 836, 776, 736, $702 \mathrm{~cm}^{-1}$.

${ }^{1} \mathbf{H}$ NMR $\left(600 \mathrm{MHz}, \mathrm{CDCl}_{3}\right): \delta 9.65(1 \mathrm{H}, \mathrm{dd}, J=1.8,3.2 \mathrm{~Hz}, \mathrm{C} 13), 7.66(4 \mathrm{H}, \mathrm{m}, \mathrm{ArH}), 7.40$ $(2 \mathrm{H}, \mathrm{m}, \operatorname{ArH}), 7.34$ (4H, m, ArH), $7.30(2 \mathrm{H}, \mathrm{m}, \operatorname{ArH}), 7.25(3 \mathrm{H}, \mathrm{m}, \mathrm{ArH}), 4.35(1 \mathrm{H}, \mathrm{d}, J=12.0$ $\left.\mathrm{Hz},-\mathrm{CH}_{2} \mathrm{Ph}\right), 4.32\left(1 \mathrm{H}, \mathrm{d}, J=12.0 \mathrm{~Hz},-\mathrm{CH}_{2} \mathrm{Ph}\right), 4.29(1 \mathrm{H}, \mathrm{ddt}, J=6.7,7.3,4.9 \mathrm{~Hz}, \mathrm{C} 11), 4.07$ $(1 \mathrm{H}, \mathrm{ddt}, J=6.8,10.9,5.6 \mathrm{~Hz}, \mathrm{C} 3), 3.45(2 \mathrm{H}, \mathrm{m}, \mathrm{C} 1), 3.19(1 \mathrm{H}$, dddd, $J=2.0,3.5,8.3,11.2 \mathrm{~Hz}$, C5), 3.16 (1H, dddd, $J=2.1,4.2,8.1,10.5 \mathrm{~Hz}, \mathrm{C} 9), 2.43$ (1H, dd, $J=1.8,4.8 \mathrm{~Hz}, \mathrm{C} 12), 2.34$ $(1 \mathrm{H}, \mathrm{dd}, J=1.8,4.8 \mathrm{~Hz}, \mathrm{C} 12), 1.78(2 \mathrm{H}, \mathrm{dt}, J=5.8,6.8 \mathrm{~Hz}, \mathrm{C} 2), 1.72(1 \mathrm{H}, \mathrm{ddd}, J=5.0,8.2$, $14.0 \mathrm{~Hz}, \mathrm{C} 10), 1.69$ (1H, m, C7), $1.61(1 \mathrm{H}, \mathrm{ddd}, J=5.1,8.3,14.1 \mathrm{~Hz}, \mathrm{C} 4), 1.53$ (1H, ddd, $J=$ 3.7, 6.9, $14.1 \mathrm{~Hz}, \mathrm{C} 4), 1.48(1 \mathrm{H}$, ddd, $J=4.4,7.6,13.9 \mathrm{~Hz}, \mathrm{C} 10), 1.43(1 \mathrm{H}$, brd, $J=13.0 \mathrm{~Hz}$, C8), $1.30(1 \mathrm{H}, \mathrm{qt}, J=13.3,4.1 \mathrm{~Hz}, \mathrm{C} 7), 1.25(1 \mathrm{H}$, brd, $J=14.3 \mathrm{~Hz}, \mathrm{C} 6), 1.09(1 \mathrm{H}, \mathrm{ddt}, J=3.8$, $11.3,13.3 \mathrm{~Hz}, \mathrm{C} 8), 1.02(9 \mathrm{H}, \mathrm{s}, t-\mathrm{Bu}), 0.97(1 \mathrm{H}, \mathrm{ddt}, J=4.8,11.3,13.1 \mathrm{~Hz}, \mathrm{C} 6), 0.84(9 \mathrm{H}, \mathrm{s}, t-$ $\mathrm{Bu}), 0.02(6 \mathrm{H}, \mathrm{s},-\mathrm{OTBDMS})$.

${ }^{13}$ C NMR (125 MHz, $\left.\mathrm{CDCl}_{3}\right): \delta 202.5,138.5,135.87$ (2C), 135.84 (2C), 134.5, 134.4, 129.51, 125.49, 128.2 (2C), 127.6 (2C), 127.51 (2C), 127.47 (2C), 127.37, 74.6, 73.7, 72.7, 68.9, 66.9, 65.3, 50.4, 44.8, 44.2, 37.9, 31.7, 31.6, 27.0 (3C), 25.7 (3C), 23.5, 19.4, 17.9, -4.4, -4.8.

HRMS (FAB+): Calculated for $\mathrm{C}_{42} \mathrm{H}_{62} \mathrm{O}_{5} \mathrm{NaSi}_{2}[\mathrm{M}+\mathrm{Na}]:$ 725.4034. Found: 725.4035.

$[\alpha]_{D}^{22}=+10.8^{\circ}\left(c=2.13, \mathrm{CHCl}_{3}\right)$. 

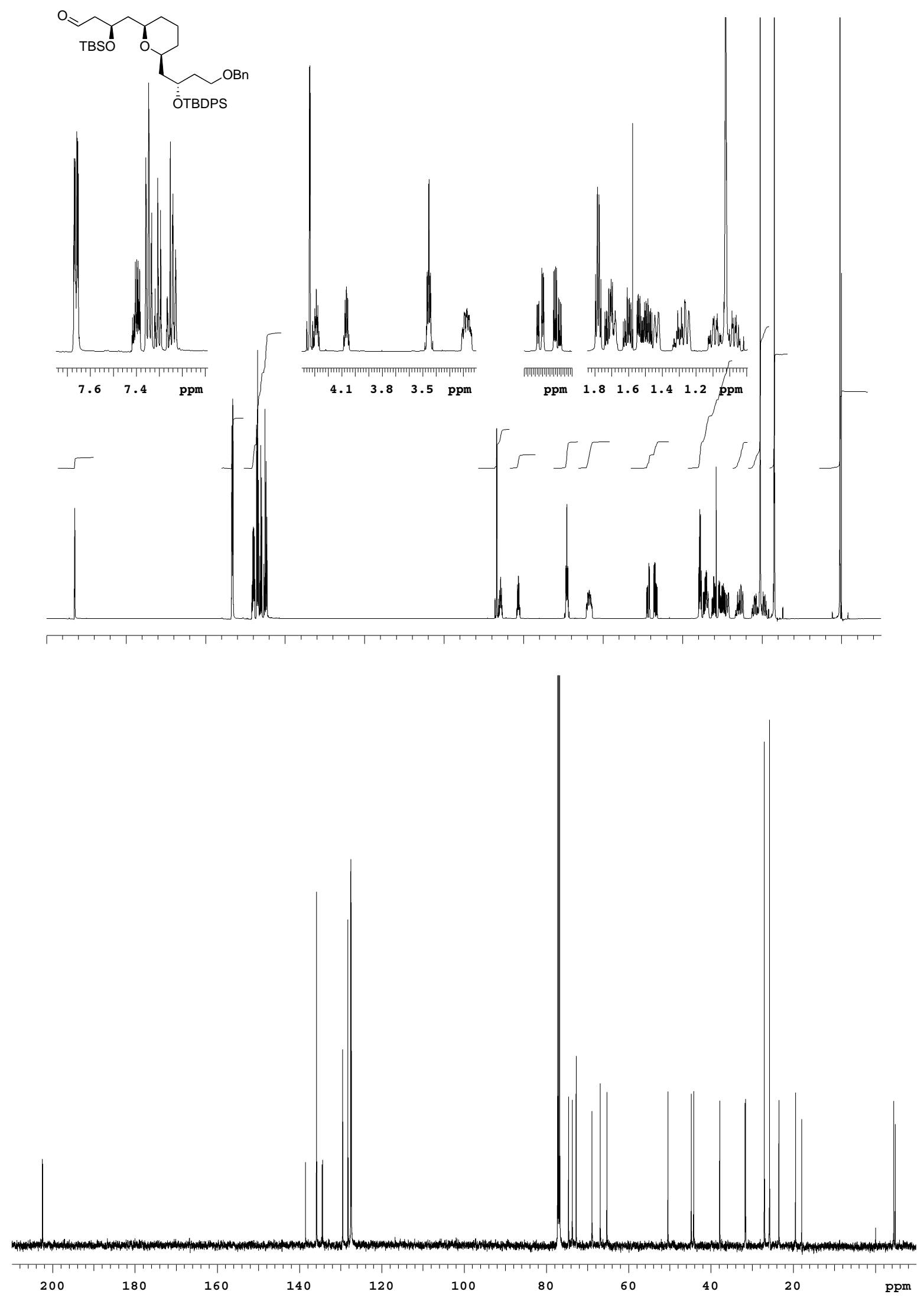


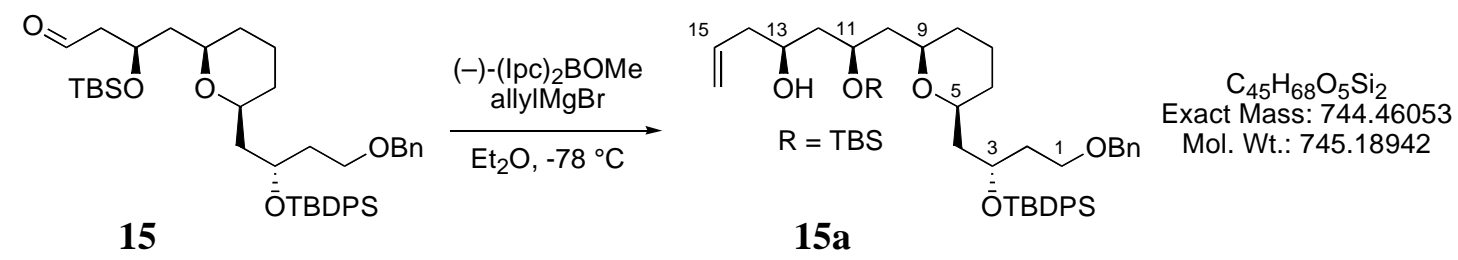

To a solution of (-)-(Ipc) $)_{2} \mathrm{BOMe}(103 \mathrm{mg}, 0.292 \mathrm{mmol}$, weighed under an inert atmosphere) in $\mathrm{Et}_{2} \mathrm{O}(1.3 \mathrm{~mL})$ under $\mathrm{N}_{2}$ at $-78{ }^{\circ} \mathrm{C}$ (external temp.), was added allylmagnesium bromide (1.0 $\mathrm{M}$ in $\mathrm{Et}_{2} \mathrm{O}, 0.24 \mathrm{~mL}, 0.24 \mathrm{mmol}$ ) in a single portion via syringe. The bath was removed and the solution stirred for $1 \mathrm{~h}$ while it warmed to rt. The flask was cooled back to $-78{ }^{\circ} \mathrm{C}$ (external temp.) and aldehyde $15(114 \mathrm{mg}, 0.162 \mathrm{mmol})$, as a solution in $2.9 \mathrm{~mL} \mathrm{Et}_{2} \mathrm{O}$, was added in a single portion via cannula. The suspension was stirred for $2.5 \mathrm{~h}$ at $-78{ }^{\circ} \mathrm{C}$. The cold bath was removed and $\mathrm{H}_{2} \mathrm{O}_{2}(1.25 \mathrm{~mL}$ of a $30 \%$ aq. solution) and $\mathrm{NaOH}(0.4 \mathrm{~mL}$ of a $1 \mathrm{~N}$ aq. solution) were added via syringe. The biphasic mixture was allowed to gently reflux in a warm water bath for $30 \mathrm{~min}$. The mixture was diluted with water $(2 \mathrm{~mL})$ and $\mathrm{Na}_{2} \mathrm{~S}_{2} \mathrm{O}_{3}(3 \mathrm{~mL}$ of a sat. aq. solution) and extracted with $\mathrm{Et}_{2} \mathrm{O}(3 \times 5 \mathrm{~mL})$. The combined organic layers were dried over anhydrous $\mathrm{MgSO}_{4}$, filtered and concentrated in vacuo. The resulting residue was dissolved in $\mathrm{MeOH}$ and evaporated to dryness $(3 \times 5 \mathrm{~mL})$ and then chromatographed on silica ( $15 \%$ EtOAc / pentane) to give $82.7 \mathrm{mg}$ of alcohol 15a as a colorless oil $(69 \%)$.

Data for 15a:

$\mathbf{R}_{f}=0.46$ (15\% EtOAc / hexanes ether) — one black spot w/ p-anisaldehyde stain.

IR (thin film): 3468, 3071, 2930, 2857, 1641, 1589, 1472, 1428, 1389, 1361, 1332, 1256, 1199 , $1111,1005,938,912,837,823,776,736,701 \mathrm{~cm}^{-1}$.

${ }^{1} \mathbf{H}$ NMR (600 MHz, CDCl $): \delta 7.66(4 \mathrm{H}, \mathrm{m}, \mathrm{ArH}), 7.40(2 \mathrm{H}, \mathrm{m}, \mathrm{ArH}), 7.35$ (4H, m, ArH), 7.31 $(2 \mathrm{H}, \mathrm{m}, \operatorname{ArH}), 7.25(3 \mathrm{H}, \mathrm{m}, \mathrm{ArH}), 5.80(1 \mathrm{H}, \mathrm{ddt}, J=10.3,17.4,7.1 \mathrm{~Hz}, \mathrm{C} 15), 5.07(2 \mathrm{H}, \mathrm{m}$, C16), $4.35\left(1 \mathrm{H}, \mathrm{d}, J=12.0 \mathrm{~Hz},-\mathbf{C H}_{2} \mathrm{Ph}\right), 4.33\left(1 \mathrm{H}, \mathrm{d}, J=12.0 \mathrm{~Hz},-\mathrm{CH}_{2} \mathrm{Ph}\right), 4.05(2 \mathrm{H}, \mathrm{m}, \mathrm{C} 3 \&$ C11), 3.77 (1H, ttd, $J=2.1,6.3,10.8 \mathrm{~Hz}, \mathrm{C} 13), 3.46(2 \mathrm{H}, \mathrm{m}, \mathrm{C} 1), 3.21(1 \mathrm{H}, \mathrm{d}, J=2.1 \mathrm{~Hz}, \mathrm{C} 13-$ OH), 3.16 (2H, m, C5 \& C9), 2.17 (2H, m, C14), 1.78 (2H, m, C2), 1.67 (2H, m, C7 \& C10), $1.64(1 \mathrm{H}, \mathrm{ddd}, J=2.3,4.7,14.0 \mathrm{~Hz}, \mathrm{C} 12), 1.61(1 \mathrm{H}, \mathrm{ddd}, J=5.5,8.1,14.0 \mathrm{~Hz}, \mathrm{C} 4), 1.52(1 \mathrm{H}$, ddd, $J=3.8,6.8,14.2 \mathrm{~Hz}, \mathrm{C} 4), 1.47(2 \mathrm{H}, \mathrm{m}, \mathrm{C} 10 \& \mathrm{C} 12), 1.43(1 \mathrm{H}, \mathrm{brd}, J=13.1 \mathrm{~Hz}, \mathrm{C} 8), 1.30$ $(1 \mathrm{H}, \mathrm{qt}, J=13.1,3.9 \mathrm{~Hz}, \mathrm{C} 7), 1.23(1 \mathrm{H}, \mathrm{brd}, J=13.3 \mathrm{~Hz}, \mathrm{C} 6), 1.07$ (1H, ddt, $J=3.9,11.0,12.9$ $\mathrm{Hz}, \mathrm{C} 8), 1.02(9 \mathrm{H}, \mathrm{s}, t-\mathrm{Bu}), 0.96(1 \mathrm{H}, \mathrm{ddt}, J=4.1,11.3,13.1 \mathrm{~Hz}, \mathrm{C} 6), 0.84(9 \mathrm{H}, \mathrm{s}, t-\mathrm{Bu}), 0.07$ (3H, s, -OTBDMS), 0.05 (3H, s, -OTBDMS).

${ }^{13}$ C NMR (125 MHz, $\left.\mathrm{CDCl}_{3}\right): \delta 138.5,135.84(2 \mathrm{C}), 135.81$ (2C), 135.0, 134.6, 134.4, 129.49, 129.48, 128.2 (2C), $127.6(2 \mathrm{C}), 127.50$ (2C), 127.48 (2C), 127.36, 117.3, 74.5, 74.1, 72.8, 69.9, 69.8, 69.0, 66.9, 45.0, 44.8, 42.7, 42.2, 37.7, 31.79, 31.78, 27.0 (3C), $25.8(3 \mathrm{C}), 23.5,19.5,17.8$, $-4.1,-4.7$.

HRMS (FAB+): Calculated for $\mathrm{C}_{45} \mathrm{H}_{69} \mathrm{O}_{5} \mathrm{Si}_{2}[\mathrm{M}+\mathrm{H}]$ : 745.4684. Found: 745.4685 . $[\alpha]_{D}^{24}=-2.56^{\circ}\left(c=2.60, \mathrm{CHCl}_{3}\right)$. 

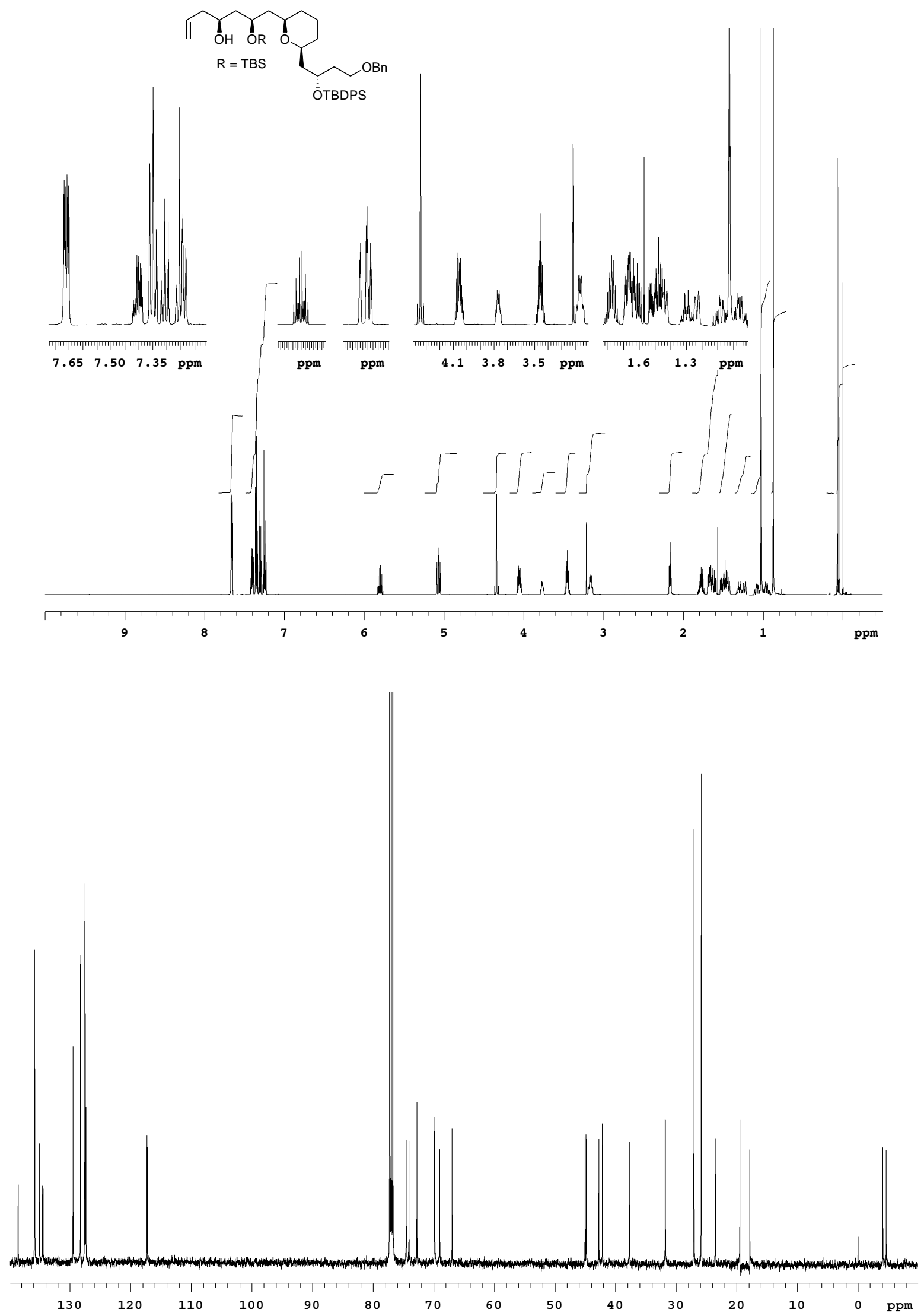


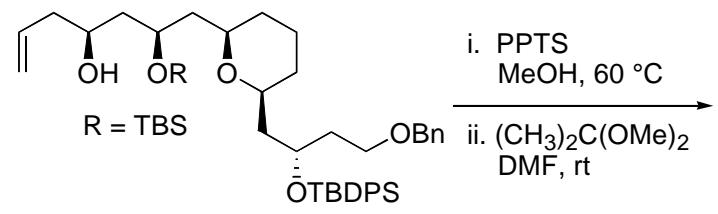

$15 a$

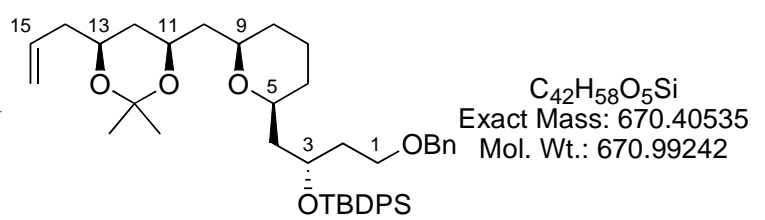

15b

A solution of alcohol 15a (17.4 mg, $0.0233 \mathrm{mmol})$ and PPTS (6 mg, $0.02 \mathrm{mmol})$ in $\mathrm{MeOH}$ $(0.83 \mathrm{~mL})$ was heated at $60{ }^{\circ} \mathrm{C}$ for $1.8 \mathrm{~h}$ and then the solvent was removed under a stream of $\mathrm{N}_{2}$ gas. Residual $\mathrm{MeOH}$ was removed under high-vacuum and the residue was redissolved in DMF $(0.83 \mathrm{~mL})$. 2,2-dimethoxypropane $(0.20 \mathrm{~mL}, 1.6 \mathrm{mmol})$ was added in a single portion via syringe and the reaction was stirred for $2.5 \mathrm{~h}$ at $\mathrm{rt}$. The reaction mixture was diluted with water $(1 \mathrm{~mL})$ and sat. aq. $\mathrm{NaHCO}_{3}(2 \mathrm{~mL})$ and extracted with $\mathrm{Et}_{2} \mathrm{O}(3 \times 3 \mathrm{~mL})$. The combined organic layers were dried over anhydrous $\mathrm{MgSO}_{4}$, filtered and concentrated in vacuo. Chromatography on silica (10\% EtOAc / pentane) gave $14.1 \mathrm{mg}$ of acetonide $\mathbf{1 5 b}$ as a colorless liquid $(90 \%)$.

Data for 15b:

$\mathbf{R}_{f}=0.33$ (10\% EtOAc / hexanes ether) — one black spot w/ p-anisaldehyde stain.

IR (thin film): 3070, 2991, 2934, 2857, 1642, 1589, 1472, 1454, 1428, 1378, 1264, 1200, 1173, $1111,1046,946,915,822,735,701 \mathrm{~cm}^{-1}$.

${ }^{1} \mathbf{H}$ NMR (600 MHz, CDCl 3$): \delta 7.67$ (4H, m, ArH), 7.40 (2H, m, ArH), 7.35 (4H, m, ArH), 7.31 $(2 \mathrm{H}, \mathrm{m}, \operatorname{ArH}), 7.25(3 \mathrm{H}, \mathrm{m}, \mathrm{ArH}), 5.78(1 \mathrm{H}, \mathrm{dddd}, J=6.6,7.4,10.3,17.1 \mathrm{~Hz}, \mathrm{C} 15), 5.07(1 \mathrm{H}$, ddt, $J=2.0,17.1,1.5 \mathrm{~Hz}, \mathrm{C} 16), 5.04$ (1H, ddt, $J=2.2,10.3,1.1 \mathrm{~Hz}, \mathrm{C} 16), 4.34\left(2 \mathrm{H}, \mathrm{s},-\mathrm{CH}_{2} \mathrm{Ph}\right)$, $4.09(1 \mathrm{H}, \mathrm{dq}, J=5.5,6.8 \mathrm{~Hz}, \mathrm{C} 3), 3.93(1 \mathrm{H}, \mathrm{ddt}, J=2.3,11.4,6.7 \mathrm{~Hz}, \mathrm{C} 11), 3.83(1 \mathrm{H}, \mathrm{ddt}, J=$ 2.6, 11.7, $6.2 \mathrm{~Hz}, \mathrm{C} 13), 3.48$ (1H, ddd, $J=6.5,7.2,16.4 \mathrm{~Hz}, \mathrm{C} 1), 3.45$ (1H, ddd, $J=6.9,7.3$, $16.4 \mathrm{~Hz}, \mathrm{C} 1), 3.22$ (2H, m, C5 \& C9), 2.27 (1H, dtt, J=14.0, 1.4, $6.3 \mathrm{~Hz}, \mathrm{C} 14), 2.12$ (1H, dddt, $J=6.3,7.6,14.0,1.3 \mathrm{~Hz}, \mathrm{C} 14), 1.78(2 \mathrm{H}, \mathrm{m}, \mathrm{C} 2), 1.72(1 \mathrm{H}, \mathrm{dt}, J=13.8,6.9 \mathrm{~Hz}, \mathrm{C} 10), 1.69(1 \mathrm{H}$, m, C7), 1.61 (1H, ddd, $J=5.4,8.6,14.1 \mathrm{~Hz}, \mathrm{C} 4), 1.52(1 \mathrm{H}, \mathrm{ddd}, J=3.4,6.9,14.1 \mathrm{~Hz}, \mathrm{C} 4) .1 .49$ $(1 \mathrm{H}$, brd, $J=13.0, \mathrm{C} 8), 1.45(1 \mathrm{H}, \mathrm{dt}, J=12.8,2.4 \mathrm{~Hz}, \mathrm{C} 12), 1.38\left(6 \mathrm{H}, \mathrm{s},-\mathrm{C}\left(\mathrm{CH}_{3}\right)_{2}\right), 1.33(2 \mathrm{H}$, m, C7 \& C10), $1.25(1 \mathrm{H}$, brd, $J=13.7 \mathrm{~Hz}, \mathrm{C} 6), 1.08(2 \mathrm{H}, \mathrm{m}, \mathrm{C} 8 \& \mathrm{C} 12), 1.03(9 \mathrm{H}, \mathrm{s},-$ OTBDMS), 0.99 (1H, ddt, $J=4.2,11.1,13.1 \mathrm{~Hz}, \mathrm{C} 6)$.

${ }^{13}$ C NMR (125 MHz, $\mathrm{CDCl}_{3}$ ): $\delta$ 138.6, 138.89 (2C), 135.87 (2C), 134.5, 134.3, 129.48, 129.45, 128.2 (2C), 127.55 (2C), 127.50 (2C), 127.47 (2C), 127.35 (2C), 117.0, 98.3, 74.5, 73.4, 72.7, 68.8, 68.5, 67.0, 65.6, 44.6, 43.1, 40.8, 37.6, 36.5, 31.9, 31.2, 30.2, 27.1 (3C), 23.5, 19.7, 19.5 .

HRMS (FAB+): Calculated for $\mathrm{C}_{42} \mathrm{H}_{58} \mathrm{O}_{5} \mathrm{NaSi}[\mathrm{M}+\mathrm{Na}]$ : 693.3951. Found: 693.3949.

$[\alpha]_{D}^{23}=+4.45^{\circ}\left(c=1.34, \mathrm{CHCl}_{3}\right)$.

Stereochemical Assignment for 15b: syn-acetonide (based on ${ }^{13} \mathrm{CNMR}$ shifts) ${ }^{\mathrm{ii}}$

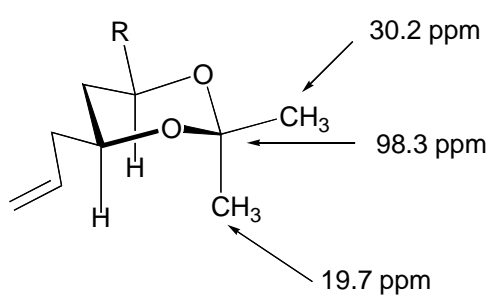



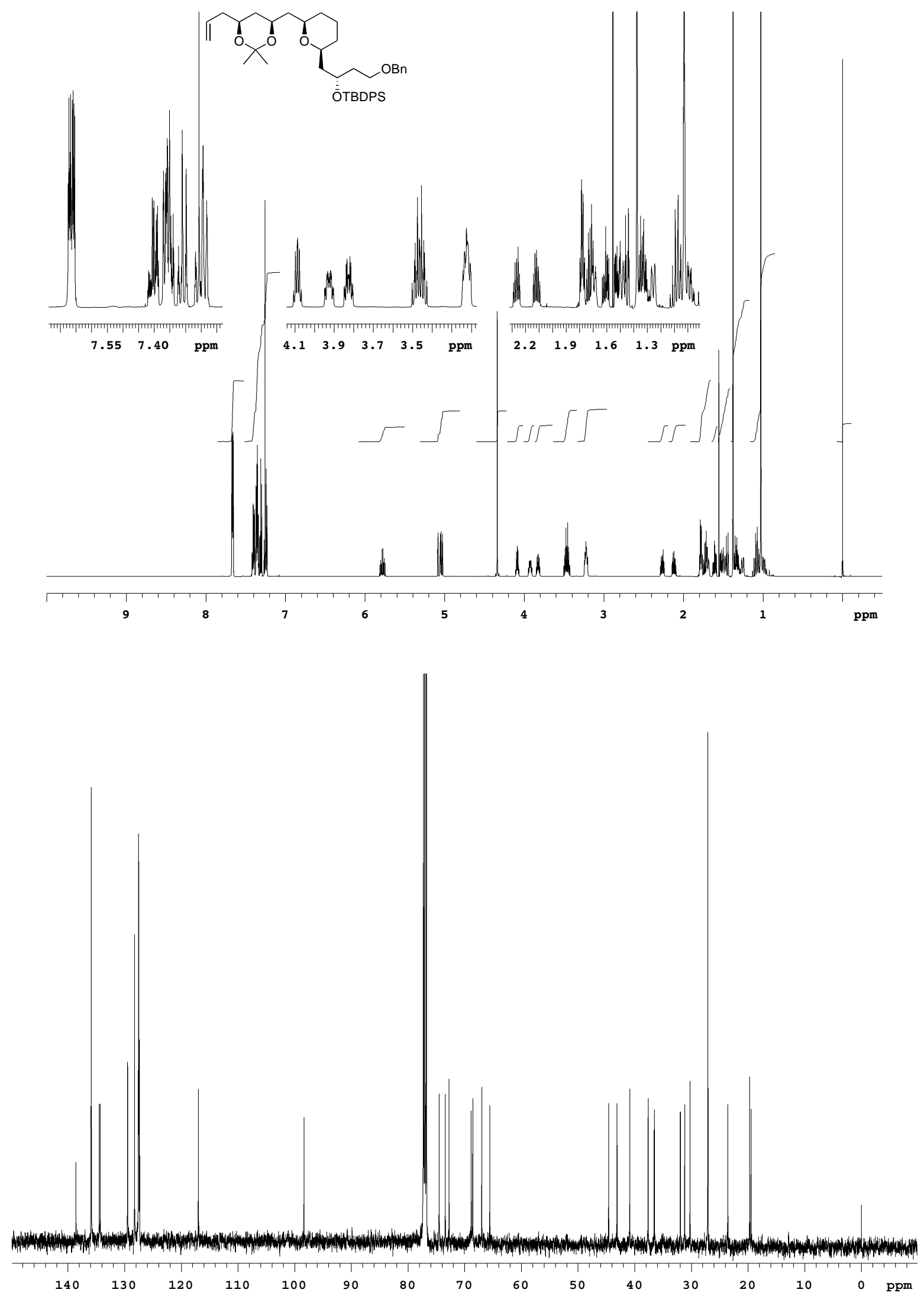


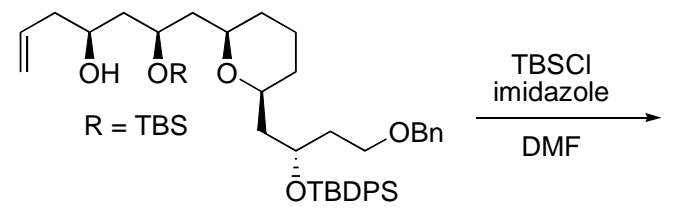

15a

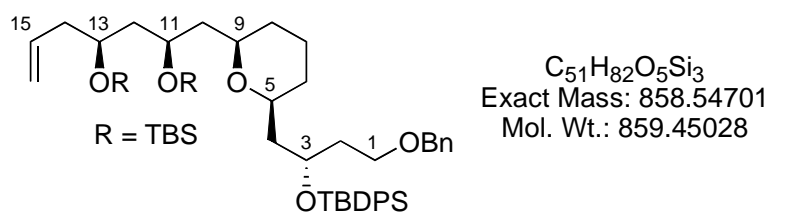

16

To a solution of alcohol 15a $(80.5 \mathrm{mg}, 0.108 \mathrm{mmol})$ in DMF $(0.5 \mathrm{~mL})$, under $\mathrm{N}_{2}$ at $\mathrm{rt}$, was added a single portion of solid imidazole $(15 \mathrm{mg}, 0.22 \mathrm{mmol})$ followed by a single portion of solid TBSCl (24 mg, $0.16 \mathrm{mmol})$. The reaction was allowed to stir for $7 \mathrm{~h}$ and was then diluted with water $(1 \mathrm{~mL})$ and sat. aq. $\mathrm{NaHCO}_{3}(2 \mathrm{~mL})$. The mixture was extracted with $\mathrm{Et}_{2} \mathrm{O}(4 \times 3 \mathrm{~mL})$ and the combined organic layers were dried over anhydrous $\mathrm{MgSO}_{4}$, filtered and concentrated in vacuo. Chromatography on silica (5\% EtOAc / pentane) gave $82.3 \mathrm{mg}$ of silyl ether 16 as a colorless oil $(89 \%)$.

Data for 16:

$\mathbf{R}_{f}=0.51$ (5\% EtOAc / hexanes ether) — one black spot w/ p-anisaldehyde stain.

IR (thin film): 3071, 2929, 1856, 1640, 1589, 1472, 1462, 1427, 1388, 1361, 1256, 1198, 1110 , $1104,1074,1047,1005,938,912,835,774,737,701 \mathrm{~cm}^{-1}$.

${ }^{1} \mathbf{H}$ NMR (600 MHz, $\left.\mathrm{CDCl}_{3}\right): \delta 7.67$ (4H, m, ArH), $7.40(2 \mathrm{H}, \mathrm{m}, \mathrm{ArH}), 7.35$ (4H, m, ArH), 7.31 $(2 \mathrm{H}, \mathrm{m}, \mathrm{ArH}), 7.25(3 \mathrm{H}, \mathrm{m}, \mathrm{ArH}), 5.79(1 \mathrm{H}, \mathrm{ddt}, J=10.6,16.6,7.2 \mathrm{~Hz}, \mathrm{C} 15), 5.03(2 \mathrm{H}, \mathrm{m}$, C16), $4.34\left(2 \mathrm{H}, \mathrm{s},-\mathrm{CH}_{2} \mathrm{Ph}\right), 4.06(1 \mathrm{H}, \mathrm{dq}, J=6.9,5.6 \mathrm{~Hz}, \mathrm{C} 3), 3.85(1 \mathrm{H}, \mathrm{tt}, J=5.4,7.1 \mathrm{~Hz}$, C11), $3.77(1 \mathrm{H}, \mathrm{dq}, J=7.3,5.3 \mathrm{~Hz}, \mathrm{C} 13), 3.50(1 \mathrm{H}, \mathrm{ddd}, J=6.0,7.7,9.2 \mathrm{~Hz}, \mathrm{C} 1), 3.46(1 \mathrm{H}$, ddd, $J=6.6,7.7,9.2 \mathrm{~Hz}, \mathrm{C} 1), 3.31$ (1H, dddd, $J=2.0,5.9,7.5,11.1 \mathrm{~Hz}, \mathrm{C} 9), 3.27$ (1H, dddd, $J=$ 1.8, 4.2, 7.7, $11.1 \mathrm{~Hz}, \mathrm{C} 5), 2.24(1 \mathrm{H}$, dddt, $J=5.9,7.2,12.7,1.3 \mathrm{~Hz}, \mathrm{C} 14), 2.14(1 \mathrm{H}$, dddt, $J=$ 5.3, 7.6, 12.7, 1.3 Hz, C14), 1.77 (2H, m, C2), 1.70 (1H, m, C7), 1.52-1.66 (6H, m, C4, C8, C10 \& C12), $1.47(1 \mathrm{H}$, ddd, $J=5.2,7.3,13.5 \mathrm{~Hz}, \mathrm{C} 10), 1.30(2 \mathrm{H}, \mathrm{m}, \mathrm{C} 6$ \& C7), 1.03 (1H, m, C8), $1.03(9 \mathrm{H}, \mathrm{s}, t-\mathrm{Bu}), 0.97(1 \mathrm{H}, \mathrm{m}, \mathrm{C} 6), 0.89(9 \mathrm{H}, \mathrm{s}, t-\mathrm{Bu}), 0.87(9 \mathrm{H}, \mathrm{s}, t-\mathrm{Bu}), 0.05(6 \mathrm{H}, \mathrm{s}$, OTBDMS), 0.04 (3H, s, -OTBDMS), 0.01 (3H, s, -OTBDMS).

${ }^{13}$ C NMR (125 MHz, $\left.\mathrm{CDCl}_{3}\right): \delta 138.6,135.9$ (3C), 134.9, 134.42, 134.40, 129.5, 129.4, 128.2 (3C), $127.6(2 \mathrm{C}), 127.52$ (2C), 127.49 (2C), 127.3, 117.0, 74.6, 74.5, 72.7, 69.0, 68.6, 66.9, 66.6, 45.5, 44.8, 43.9, 42.2, 37.5, 31.9, 31.2, 27.1 (3C), 25.92 (3C), 25.89 (3C), 23.5, 19.4, 18.01, $17.99,-4.17,-4.24,-4.3,-4.4$.

HRMS (FAB+): Calculated for $\mathrm{C}_{51} \mathrm{H}_{82} \mathrm{O}_{5} \mathrm{NaSi}_{3}[\mathrm{M}+\mathrm{Na}]$ : 881.5368. Found: 881.5334.

$[\alpha]_{D}^{24}=+19.43^{\circ}\left(c=0.53, \mathrm{CHCl}_{3}\right)$. 

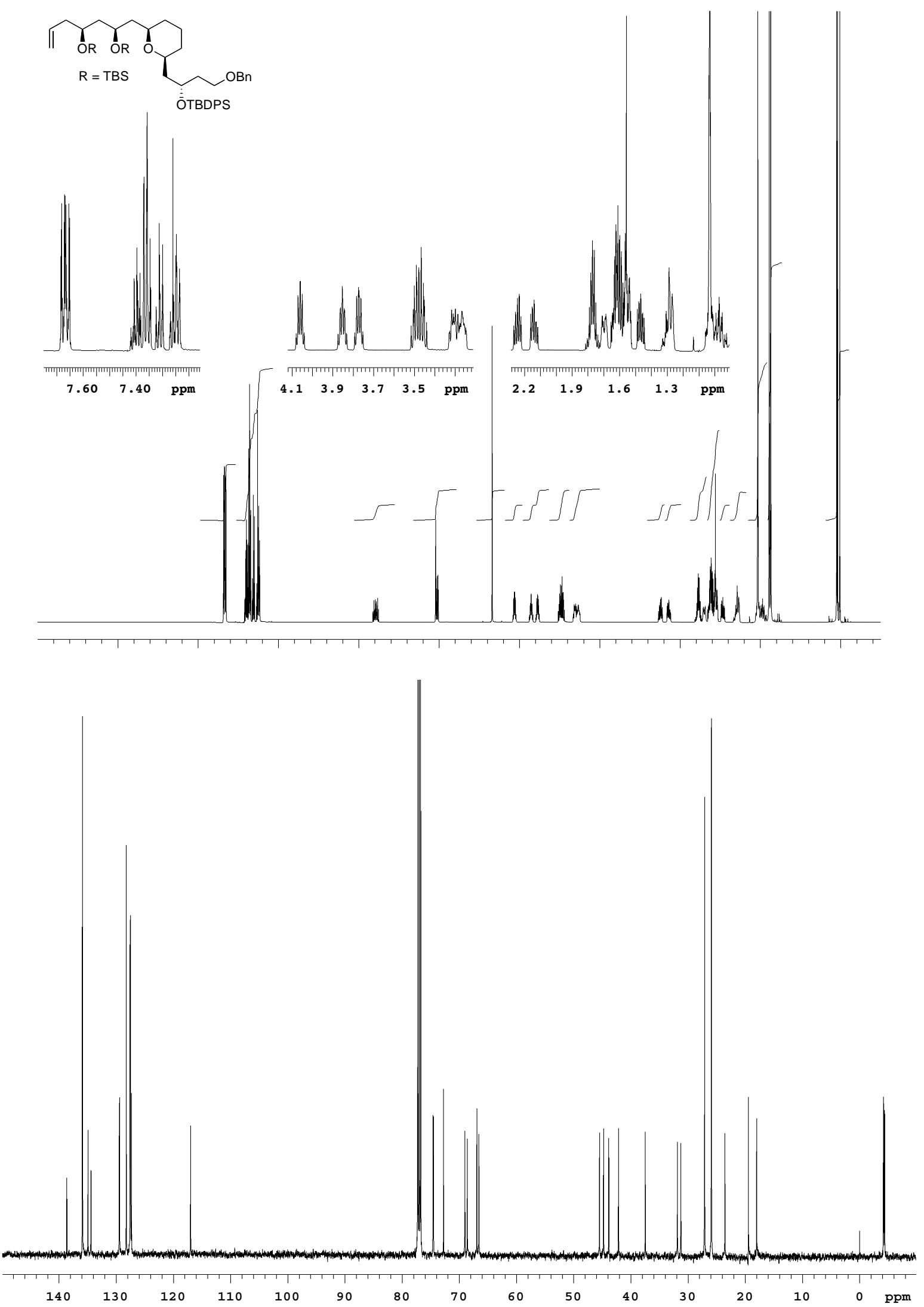


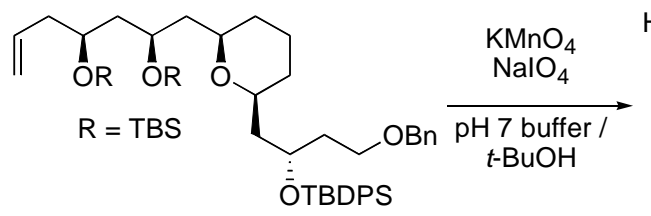

16

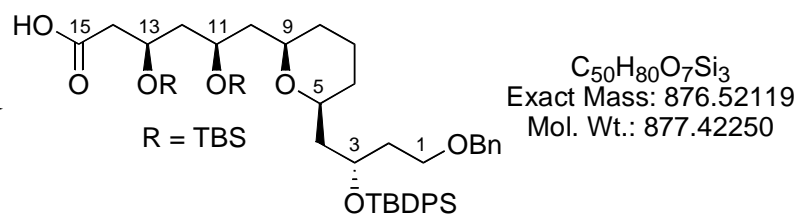

16a

Solid $\mathrm{NaIO}_{4}(35.8 \mathrm{mg}, 0.168 \mathrm{mmol})$ and $\mathrm{KMnO}_{4}(3.3 \mathrm{mg}, 0.021 \mathrm{mmol})$ were suspended in $0.86 \mathrm{~mL}$ of $\mathrm{pH} 7$ phosphate buffer and stirred at $\mathrm{rt}$ for $10 \mathrm{~min}$. This purple suspension was then directly poured into a solution of the terminal alkene $16(18.0 \mathrm{mg}, 0.021 \mathrm{mmol})$ in $t$-BuOH $(1.5$ $\mathrm{mL}$ ) using $0.44 \mathrm{~mL}$ phosphate buffer to rinse the remaining suspension into the reaction flask. The mixture was allowed to stir for $45 \mathrm{~min}$ and then diluted with sat. aq. $\mathrm{Na}_{2} \mathrm{~S}_{2} \mathrm{O}_{3}(1 \mathrm{~mL})$ and brine $(2 \mathrm{~mL})$. After extraction with $\mathrm{Et}_{2} \mathrm{O}(3 \times 3 \mathrm{~mL})$ the combined organic layers were dried over anhydrous $\mathrm{MgSO}_{4}$, filtered and concentrated. Chromatography on silica $(10 \%$ EtOAc / pentane + $0.5 \% \mathrm{AcOH})$ gave $12.4 \mathrm{mg}$ of carboxylic acid 16a as a colorless oil (67\%).

Data for 16a:

$\mathbf{R}_{f}=0.67$ (20\% EtOAc / hexanes ether) — one black spot w/ p-anisaldehyde stain.

IR (thin film): 3070, 2923, 2857, 1712, 1591, 1472, 1428, 1389, 1361, 1315, 1257, 1111, 1076, $1005,938,836,775,736,701 \mathrm{~cm}^{-1}$.

${ }^{1} \mathbf{H}$ NMR $\left(600 \mathrm{MHz}, \mathrm{CDCl}_{3}\right): \delta 7.66(4 \mathrm{H}, \mathrm{m}, \mathrm{ArH}), 7.40(2 \mathrm{H}, \mathrm{m}, \operatorname{ArH}), 7.35(4 \mathrm{H}, \mathrm{m}, \mathrm{ArH}), 7.30$ $(2 \mathrm{H}, \mathrm{m}, \mathrm{ArH}), 7.26(3 \mathrm{H}, \mathrm{m}, \mathrm{ArH}), 4.37\left(1 \mathrm{H}, \mathrm{d}, J=12.1 \mathrm{~Hz},-\mathrm{CH}_{2} \mathrm{Ph}\right), 4.35(1 \mathrm{H}, \mathrm{d}, J=12.1 \mathrm{~Hz}$, $\left.\mathrm{CH}_{2} \mathrm{Ph}\right), 4.21(1 \mathrm{H}, \mathrm{dq}, J=7.4,5.6 \mathrm{~Hz}, \mathrm{C} 13), 4.02(1 \mathrm{H}, \mathrm{dq}, J=4.8,6.4 \mathrm{~Hz}, \mathrm{C} 3), 3.82(1 \mathrm{H}, \mathrm{tt}, J=$ 5.1, 7.1 Hz, C11), 3.54 (1H, ddd, $J=5.5,7.6,9.3 \mathrm{~Hz}, \mathrm{C} 1), 3.50(1 \mathrm{H}, \mathrm{dt}, J=9.3,7.1 \mathrm{~Hz}, \mathrm{C} 1)$, 3.21 (1H, dddd, $J=1.9,5.0,7.0,11.4 \mathrm{~Hz}, \mathrm{C} 9), 3.15$ (1H, dddd, $J=2.0,4.4,7.5,11.4 \mathrm{~Hz}, \mathrm{C} 5)$, $2.55(1 \mathrm{H}, \mathrm{dd}, J=5.5,15.1 \mathrm{~Hz}, \mathrm{C} 14), 2.45(1 \mathrm{H}, \mathrm{dd}, J=5.5,15.1 \mathrm{~Hz}, \mathrm{C} 14), 1.82(1 \mathrm{H}, \mathrm{ddt}, J=4.8$, 14.0, 7.0 Hz, C2), 1.60-1.79 (6H, m, C2, C4, C7, C10 \& C12), 1.51 (1H, ddd, J = 4.4, 6.3, 14.1 $\mathrm{Hz}, \mathrm{C} 4), 1.45(2 \mathrm{H}, \mathrm{m}, \mathrm{C} 8$ \& C10), $1.28(1 \mathrm{H}, \mathrm{m}, \mathrm{C} 7), 1.20(1 \mathrm{H}, \mathrm{brd}, J=13.0 \mathrm{~Hz}, \mathrm{C} 6), 1.05(1 \mathrm{H}$, m, C8), $1.02(9 \mathrm{H}, \mathrm{s}, t-\mathrm{Bu}), 0.92(1 \mathrm{H}, \mathrm{m}, \mathrm{C} 6), 0.89(9 \mathrm{H}, \mathrm{s}, t-\mathrm{Bu}), 0.87(9 \mathrm{H}, \mathrm{s}, t-\mathrm{Bu}), 0.09(3 \mathrm{H}, \mathrm{s},-$ OTBDMS), 0.08 (3H, s, -OTBDMS), 0.04 (3H, s, -OTBDMS), 0.02 (3H, s, -OTBDMS).

${ }^{13}$ C NMR (125 MHz, $\left.\mathrm{CDCl}_{3}\right): \delta 174.1,138.3,135.88$ (2C), 135.87 (2C), 134.5, 134.3, 129.5, 129.4, 128.2 (2C), 127.7 (2C), 127.52 (2C), 127.47 (2C), 127.42, 74.8, 74.2, 72.7, 69.0, 67.0, 66.8, 66.5, 45.0, 44.8, 44.1, 42.0, 37.4, 31.7, 31.6, 27.0 (3C), 25.9 (3C), 25.7 (3C), 23.5, 19.4, $17.95,17.89,-4.3,-4.4,-4.5,-4.8$.

HRMS (ESI+): Calculated for $\mathrm{C}_{50} \mathrm{H}_{80} \mathrm{O}_{7} \mathrm{NaSi}_{3}[\mathrm{M}+\mathrm{Na}]$ : 899.5110. Found: 899.5095.

$[\alpha]_{D}^{23}=+1.93^{\circ}\left(c=1.79, \mathrm{CHCl}_{3}\right)$. 

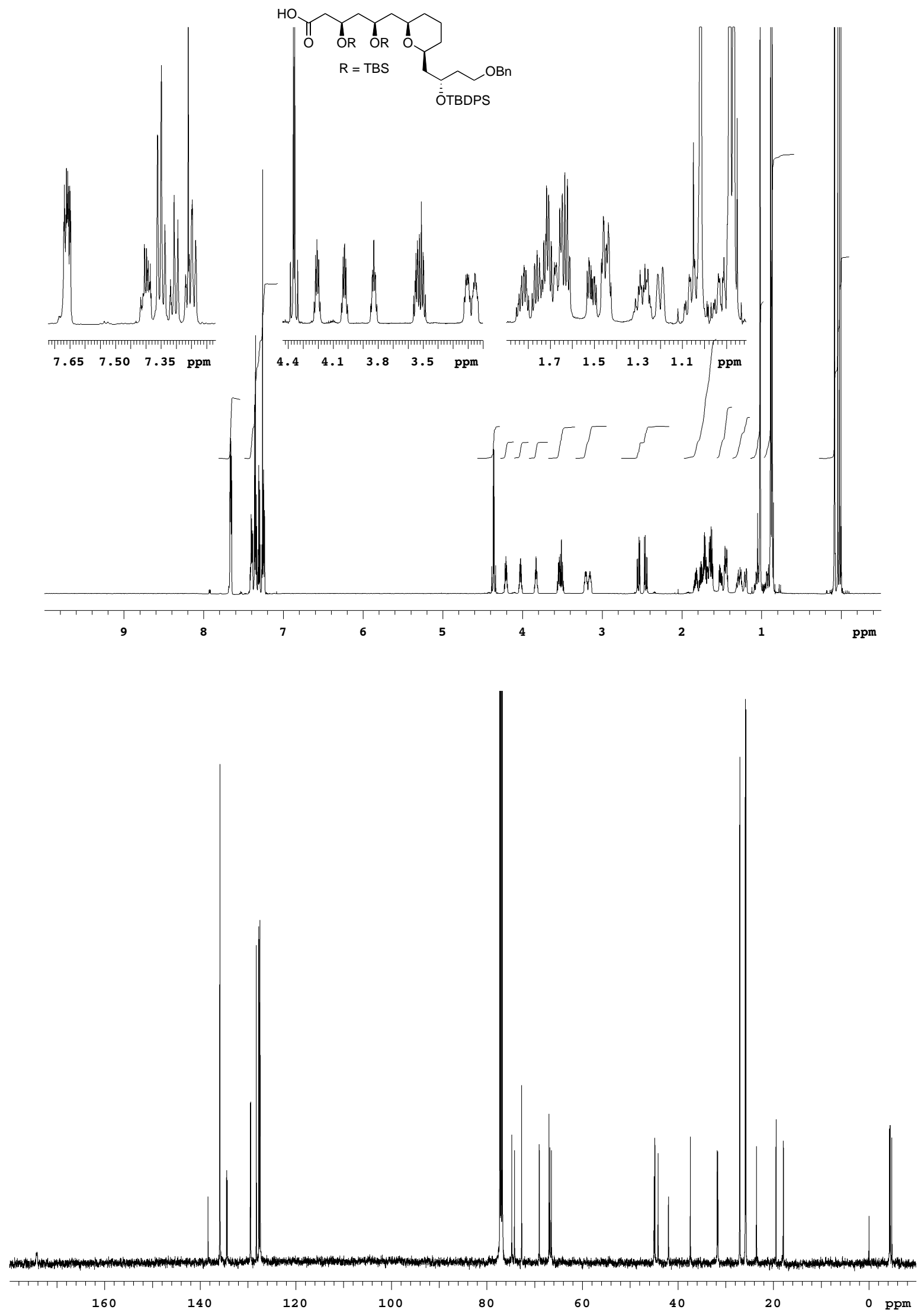


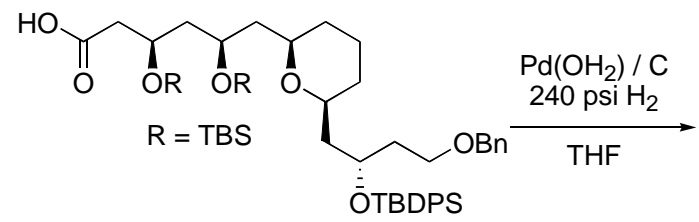

16a

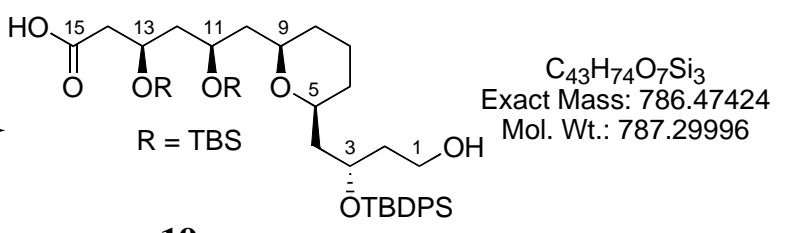

19

To a solution of benzyl ether 16a $(21.0 \mathrm{mg}, 0.0239 \mathrm{mmol})$ in THF $(2.7 \mathrm{~mL})$ was added $\mathrm{Pd}(\mathrm{OH})_{2}(20 \mathrm{wt} \% \mathrm{Pd}$ on carbon-wet, $21 \mathrm{mg})$. The reaction flask was placed in a high-pressure reaction bomb, pressurized with $\mathrm{H}_{2}(240 \mathrm{psi})$ and slowly depressurized. After repressurization with $\mathrm{H}_{2}$ (240 psi), the reaction was allowed to stir at $\mathrm{rt}$ for $3 \mathrm{~h}$. The bomb was slowly depressurized and the reaction mixture was filtered though a plug of celite using EtOAc. The filtrate was concentrated and chromatographed on silica ( $40 \%$ EtOAc / pentane $+0.5 \% \mathrm{AcOH})$ to give $17.3 \mathrm{mg}$ of alcohol 19 as a colorless oil (92\%).

Data for 18:

$\mathbf{R}_{f}=0.39$ (40\% EtOAc / petroleum ether) — one black spot w/ p-anisaldehyde stain.

IR (thin film): 3362 (br), 3071, 2930, 2857, 1713, 1591, 1471, 1428, 1389, 1257, 1105, 1081, $1049,1004,938,836,775,740,702 \mathrm{~cm}^{-1}$.

${ }^{1} \mathbf{H}$ NMR $\left(500 \mathrm{MHz}, \mathrm{CDCl}_{3}\right): \delta 7.69(4 \mathrm{H}, \mathrm{m}, \operatorname{ArH}), 7.40(6 \mathrm{H}, \mathrm{m}, \operatorname{ArH}), 4.21(1 \mathrm{H}, \mathrm{dq}, J=8.3,4.9$ $\mathrm{Hz}, \mathrm{C} 13), 4.02$ (1H, m, C3), $3.81(2 \mathrm{H}, \mathrm{m}, \mathrm{C} 1 \& \mathrm{C} 11), 3.75(1 \mathrm{H}, \mathrm{dt}, J=11.0,5.6 \mathrm{~Hz}, \mathrm{C} 1), 3.14$ (1H, m, C9), 2.92 (1H, dddd, $J=2.2,4.1,7.5,11.3 \mathrm{~Hz}, \mathrm{C} 5), 2.57$ (1H, dd, J = 5.4, $15.3 \mathrm{~Hz}, \mathrm{C} 14)$, 2.49 (1H, dd, J = 4.6, $15.3 \mathrm{~Hz}, \mathrm{C} 14), 1.89$ (1H, ddt, $J=8.2,14.2,5.0 \mathrm{~Hz}, \mathrm{C} 2), 1.60-1.79(6 \mathrm{H}, \mathrm{m}$, C2, C4, C7, C10 \& C12), 1.49 (1H, dt, $J=14.2,4.4 \mathrm{~Hz}, \mathrm{C} 4), 1.40$ (1H, ddd, $J=4.0,8.1,14.0$ $\mathrm{Hz}, \mathrm{C} 10), 1.39$ (1H, brd, $J=13.0 \mathrm{~Hz}, \mathrm{C} 8), 1.26(1 \mathrm{H}, \mathrm{qt}, J=12.3,3.8 \mathrm{~Hz}, \mathrm{C} 7), 1.07$ (2H, m, C6 \& C8), $1.05(9 \mathrm{H}, \mathrm{s}, t-\mathrm{Bu}), 0.91(1 \mathrm{H}, \mathrm{m}, \mathrm{C} 6), 0.90(9 \mathrm{H}, \mathrm{s}, t-\mathrm{Bu}), 0.87(9 \mathrm{H}, \mathrm{s}, t-\mathrm{Bu}), 0.11(6 \mathrm{H}, \mathrm{s},-$ OTBDMS), 0.05 (3H, s, -OTBDMS), 0.05 (3H, s, -OTBDMS).

${ }^{13}$ C NMR (125 MHz, $\left.\mathrm{CDCl}_{3}\right): \delta 173.5,135.91$ (2C), 135.88 (2C), 134.2, 133.8, 129.7, 129.6, 127.7 (2C), 127.6 (2C), 75.3, 74.3, 70.7, 66.8, 66.4, 59.5, 44.5, 44.4, 43.9, 41.3, 39.0, 31.75, 31.68, 27.0 (3C), 25.8 (3C), 25.7 (3C), 23.5, 19.3, 17.93, 17.89, -4.2, -4.50, -4.55, -4.9.

HRMS (ESI+): Calculated for $\mathrm{C}_{43} \mathrm{H}_{74} \mathrm{O}_{7} \mathrm{NaSi}_{3}$ [M+Na]: 809.4640. Found: 809.4645.

$[\alpha]_{D}^{25}=-9.30^{\circ}\left(c=0.95, \mathrm{CHCl}_{3}\right)$. 

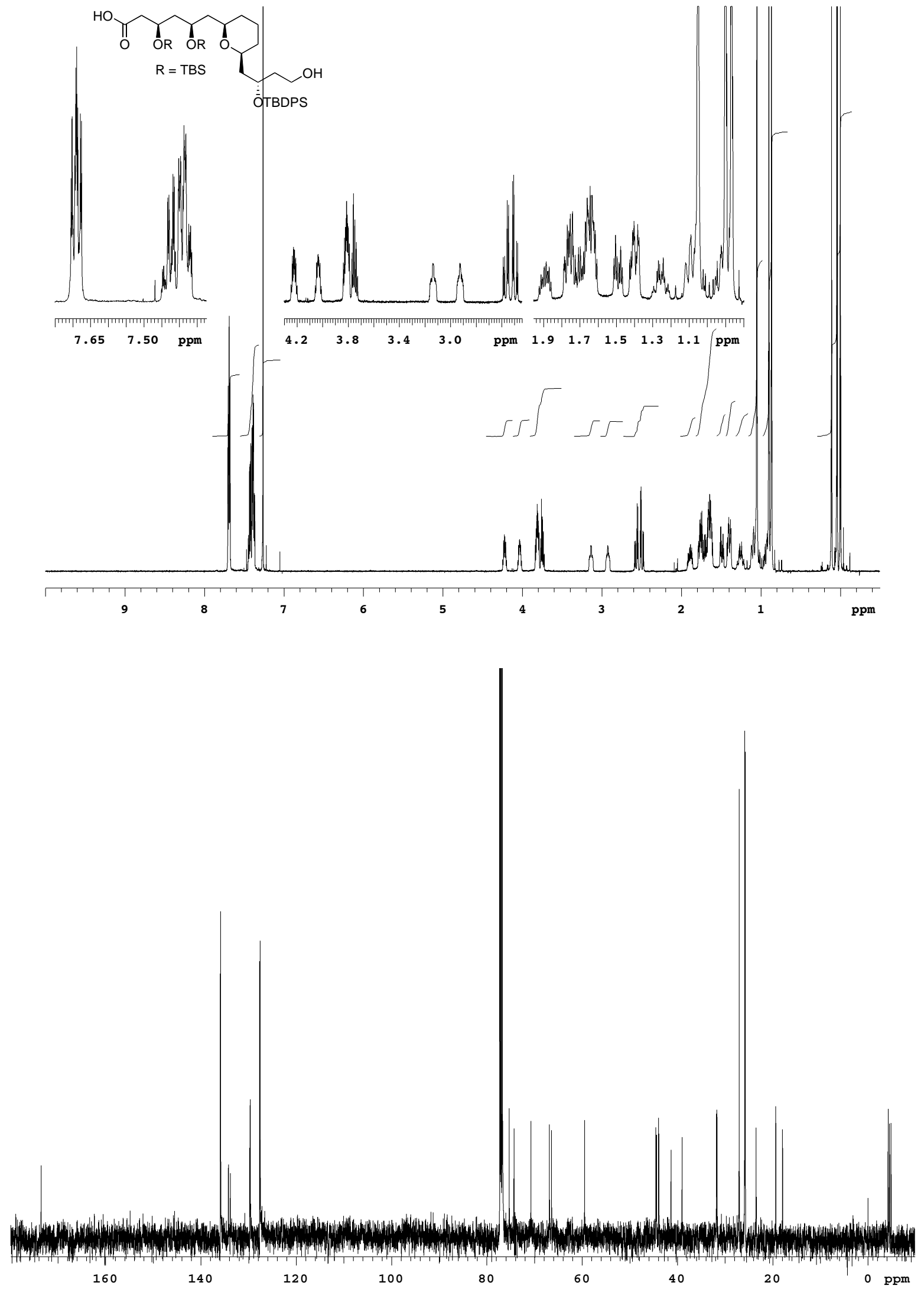


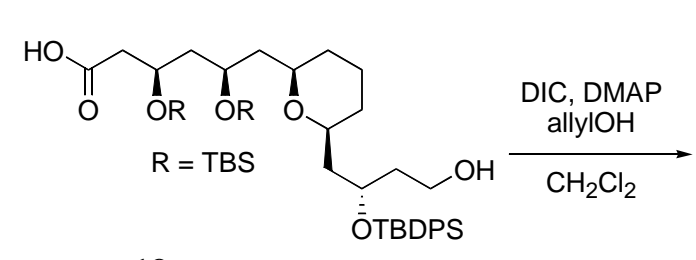

19

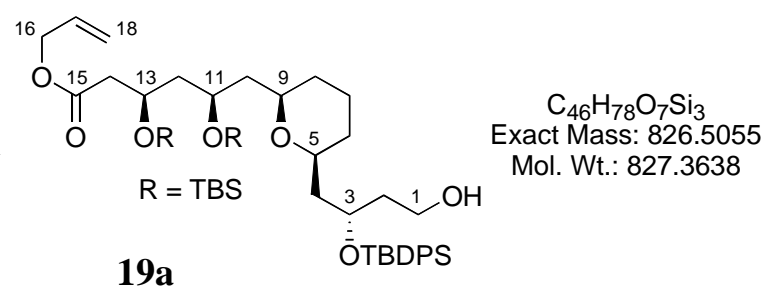

19a

To a solution of carboxylic acid $19(18.0 \mathrm{mg}, 0.0229 \mathrm{mmol})$ in $\mathrm{CH}_{2} \mathrm{Cl}_{2}(1.2 \mathrm{~mL})$, under $\mathrm{N}_{2}$ at rt, was added a single portion of solid DMAP $(4.5 \mathrm{mg}, 0.012 \mathrm{mmol})$, a single portion of allyl alcohol $(154 \mu \mathrm{L}, 2.26 \mathrm{mmol})$ via syringe and then a single portion of diisopropylcarbodiimide $(5.0 \mu \mathrm{L}, 0.032 \mathrm{mmol})$ also via syringe. The reaction was allowed to stir for $2 \mathrm{~h}$ and then was diluted with sat. aq. $\mathrm{NH}_{4} \mathrm{Cl}(2 \mathrm{~mL})$ and extracted with $\mathrm{Et}_{2} \mathrm{O}(3 \times 3 \mathrm{~mL})$. The combined organic layers were dried over anhydrous $\mathrm{MgSO}_{4}$, filtered and concentrated. Chromatography on silica (10\% EtOAc / pentane) gave $11.5 \mathrm{mg}$ of ester 19a as a colorless oil (61\%).

Data for 18a:

$\mathbf{R}_{f}=0.51$ (15\% EtOAc / petroleum ether) — one black spot $\mathrm{w} / \mathrm{p}$-anisaldehyde stain.

IR (thin film): 3464 (br), 3072, 2930, 2893, 2857, 1741, 1590, 1472, 1463, 1428, 1389, 1361, $1314,1257,1168,1131,1111,1080,1005,938,836,776,740,702 \mathrm{~cm}^{-1}$.

${ }^{1} \mathbf{H}$ NMR $\left(500 \mathrm{MHz}, \mathrm{CDCl}_{3}\right): \delta 7.69(4 \mathrm{H}, \mathrm{m}, \mathrm{ArH}), 7.41(6 \mathrm{H}, \mathrm{m}, \mathrm{ArH}), 5.91(1 \mathrm{H}, \mathrm{ddt}, J=10.5$, $17.4,5.6 \mathrm{~Hz}, \mathrm{C} 17), 5.31(1 \mathrm{H}, \mathrm{dq}, J=17.2,1.5 \mathrm{~Hz}, \mathrm{C} 18), 5.22(1 \mathrm{H}, \mathrm{dq}, J=10.4,1.3 \mathrm{~Hz}, \mathrm{C} 18)$, $4.58(1 \mathrm{H}, \mathrm{ddt}, J=5.8,13.2,1.4 \mathrm{~Hz}, \mathrm{C} 16), 4.53(1 \mathrm{H}, \mathrm{ddt}, J=5.913 .2,1.3 \mathrm{~Hz}, \mathrm{C} 16), 4.22(1 \mathrm{H}$, ddt, $J=4.55 .8,7.6 \mathrm{~Hz}, \mathrm{C} 13), 4.05(1 \mathrm{H}, \mathrm{dq}, J=7.4,4.9 \mathrm{~Hz}, \mathrm{C} 3), 3.79(2 \mathrm{H}, \mathrm{m}, \mathrm{C} 1$ \& C11), 3.71 $(1 \mathrm{H}, \mathrm{m}, \mathrm{C} 1), 3.17(1 \mathrm{H}$, dddd. $J=1.9,5.7,7.4,12.7 \mathrm{~Hz}, \mathrm{C} 9), 2.93(1 \mathrm{H}$, dddd, $J=2.2,3.7,8.4$, $10.9 \mathrm{~Hz}, \mathrm{C} 5), 2.53(1 \mathrm{H}, \mathrm{dd}, J=4.4 ., 14.5 \mathrm{~Hz}, \mathrm{C} 14), 2.39$ (1H, dd, $J=4.5,6.4 \mathrm{~Hz}, \mathrm{C} 1-\mathrm{OH}), 2.35$ $(1 \mathrm{H}, \mathrm{dd}, J=7.7,14.5 \mathrm{~Hz}, \mathrm{C} 14), 1.87(1 \mathrm{H}, \mathrm{ddt}, J=7.9,14.3,4.8 \mathrm{~Hz}, \mathrm{C} 2), 1.57-1.79(6 \mathrm{H}, \mathrm{m}, \mathrm{C} 2$, C4, C7, C10 \& C12), 1.50 (1H, ddd, $J=3.6,5.1,14.3 \mathrm{~Hz}, \mathrm{C} 4), 1.47(1 \mathrm{H}, \mathrm{m}, \mathrm{C} 8), 1.44(1 \mathrm{H}, \mathrm{ddd}$, $J=5.7,6.6,13.7 \mathrm{~Hz}, \mathrm{C} 10), 1.24(1 \mathrm{H}, \mathrm{qt}, J=13.1,4.0 \mathrm{~Hz}, \mathrm{C} 7), 1.13(1 \mathrm{H}, \mathrm{brd}, J=13.4 \mathrm{~Hz}, \mathrm{C} 6)$, $1.05(9 \mathrm{H}, \mathrm{s}, t-\mathrm{Bu}), 1.02(1 \mathrm{H}, \mathrm{m}, \mathrm{C} 8), 0.94(1 \mathrm{H}, \mathrm{m}, \mathrm{C} 6), 0.87(9 \mathrm{H}, \mathrm{s}, t-\mathrm{Bu}), 0.85(9 \mathrm{H}, \mathrm{s}, t-\mathrm{Bu})$, 0.05 (3H, s, -OTBDMS), 0.04 (3H, s, -OTBDMS), 0.02 (3H, s, -OTBDMS), 0.01 (3H, s, OTBDMS).

${ }^{13} \mathrm{C}$ NMR $\left(125 \mathrm{MHz}, \mathrm{CDCl}_{3}\right): \delta 171.2,135.88(2 \mathrm{C}), 135.85$ (2C), 134.1, 133.8, 132.2, 129.74, 129.66, 127.7 (2C), 127.6 (2C), 118.3, 75.3, 74.5, 71.1, 66.8, 66.3, 65.1, 59.5, 45.3, 44.1, 43.6, 42.6, 38.8, 31.7, 31.4, 27.0 (3C), 25.9 (3C), 25.8 (3C), 23.4, 19.3, 17.92, 17.89, -4.28, -4.32, -4.5, -4.7 .

HRMS (ESI+): Calculated for $\mathrm{C}_{46} \mathrm{H}_{78} \mathrm{O}_{7} \mathrm{NaSi}_{3}[\mathrm{M}+\mathrm{Na}]$ : 849.4953. Found: 849.4941.

$[\alpha]_{D}^{26}=-7.50^{\circ}\left(c=1.00, \mathrm{CHCl}_{3}\right)$. 

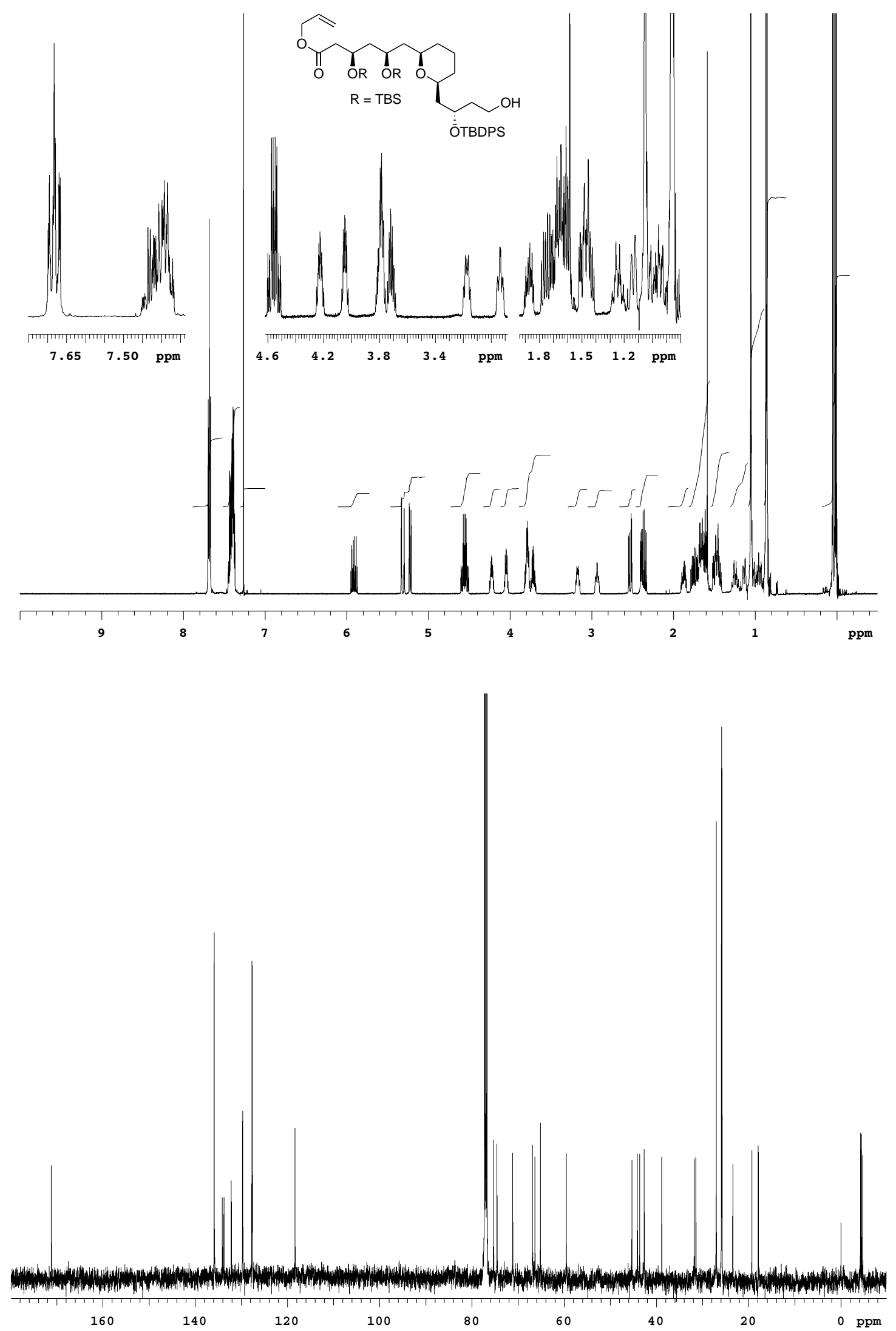


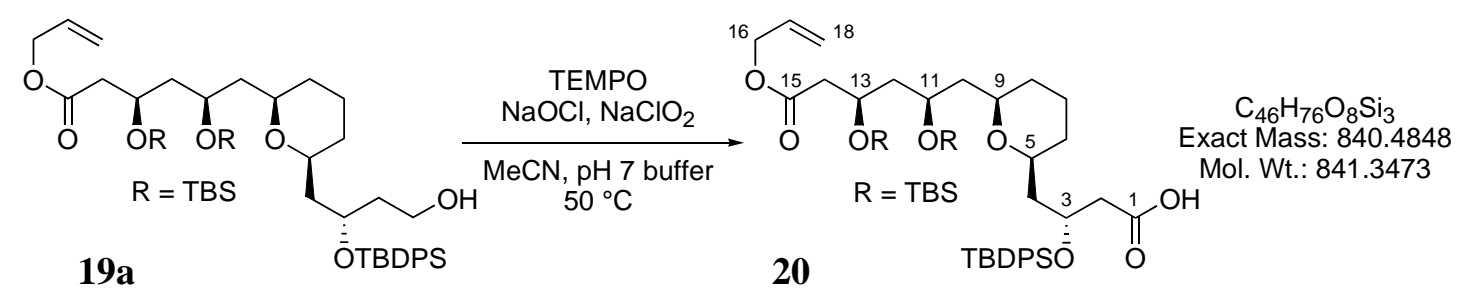

To a solution of alcohol 19a $(9.5 \mathrm{mg}, 0.012 \mathrm{mmol})$ in a mixture of $\mathrm{pH} 7$ phosphate buffer $(0.25 \mathrm{~mL})$ and $\mathrm{MeCN}(0.35 \mathrm{~mL})$, was added a single portion of solid TEMPO $(0.5 \mathrm{mg}, 0.0032$ mmol), a single portion of solid $\mathrm{NaClO}_{2}(80 \%$ technical grade, $11 \mathrm{mg}, 0.097 \mathrm{mmol})$ and a single portion of $\mathrm{NaOCl}$ (as a $5 \%$ commercial bleach solution, $3 \mu \mathrm{L}$ ) via syringe. Upon addition of the $\mathrm{NaOCl}$ solution, the reaction turned a deep purple color. The reaction was allowed to stir for $1 \mathrm{~h}$ at $50{ }^{\circ} \mathrm{C}$ and then diluted with brine $(2 \mathrm{~mL})$ and extracted with $\mathrm{Et}_{2} \mathrm{O}(4 \times 2 \mathrm{~mL})$. The combined organic layers were dried over anhydrous $\mathrm{MgSO}_{4}$, filtered and concentrated. Chromatography on silica $(15 \%$ EtOAc / pentane $+0.5 \% \mathrm{AcOH})$ gave $9.2 \mathrm{mg}$ of carboxylic acid 20 as a colorless oil $(95 \%)$.

Data for 19:

$\mathbf{R}_{f}=0.33$ (10\% EtOAc / petroleum ether) — one black spot $\mathrm{w} / \mathrm{p}$-anisaldehyde stain.

IR (thin film): 3072, 3050, 1930, 1857, 1738, 1712, 1590, 1472, 1463, 1428, 1389, 1361, 1312, $1257,1202,1168,1111,1081,1027,1005,966,938,836,808,775,740,702 \mathrm{~cm}^{-1}$.

${ }^{1} \mathbf{H}$ NMR $\left(500 \mathrm{MHz}, \mathrm{CDCl}_{3}\right): \delta 7.68(4 \mathrm{H}, \mathrm{m}, \mathrm{ArH}), 7.40(6 \mathrm{H}, \mathrm{m}, \mathrm{ArH}), 5.90(1 \mathrm{H}, \mathrm{ddt}, J=10.4$, $17.2,5.9 \mathrm{~Hz}, \mathrm{C} 17), 5.31(1 \mathrm{H}, \mathrm{dq}, J=17.2,1.6 \mathrm{~Hz}, \mathrm{C} 18), 5.22(1 \mathrm{H}, \mathrm{dq}, J=10.4,1.3 \mathrm{~Hz}, \mathrm{C} 18)$, $4.57(1 \mathrm{H}, \mathrm{ddt}, J=5.7,13.2,1.3 \mathrm{~Hz}, \mathrm{C} 16), 4.52(1 \mathrm{H}, \mathrm{ddt}, J=5.913 .2,1.3 \mathrm{~Hz}, \mathrm{C} 16), 4.21(2 \mathrm{H}, \mathrm{m}$, C3 \& C13), $3.79(1 \mathrm{H}$, pent, C11), $3.23(1 \mathrm{H}, \mathrm{ddt}, J=1.6,7.9,6.3 \mathrm{~Hz}, \mathrm{C} 11), 3.03(1 \mathrm{H}$, ddt, $J=$ 8.8, 10.9, 2.3 Hz, C9), $2.58(1 \mathrm{H}, \mathrm{dd}, J=5.3,15.0 \mathrm{~Hz}, \mathrm{C} 2$ or $\mathrm{C} 14), 2.52(2 \mathrm{H}, \mathrm{m}, \mathrm{C} 2$ or C14), 2.37 $(1 \mathrm{H}, \mathrm{dd}, J=7.3,14.5 \mathrm{~Hz}, \mathrm{C} 2$ or C14), $1.55-1.76(6 \mathrm{H}, \mathrm{m}, \mathrm{C} 4, \mathrm{C} 7, \mathrm{C} 10 \& \mathrm{C} 12), 1.51$ (1H, brd, $J=$ $13.2 \mathrm{~Hz}, \mathrm{C} 8), 1.48(1 \mathrm{H}, \mathrm{dt}, J=13.6,6.9 \mathrm{~Hz}, \mathrm{C} 10), 1.28(1 \mathrm{H}, \mathrm{qt}, J=13.4,3.8 \mathrm{~Hz}, \mathrm{C} 7), 1.21(1 \mathrm{H}$, brd, $J=13.4 \mathrm{~Hz}, \mathrm{C} 6), 1.04(9 \mathrm{H}, \mathrm{s}, t-\mathrm{Bu}), 1.03(2 \mathrm{H}, \mathrm{m}, \mathrm{C} 6 \& \mathrm{C} 8), 0.86(9 \mathrm{H}, \mathrm{s}, t-\mathrm{Bu}), 0.85(9 \mathrm{H}, \mathrm{s}$, $t$-Bu), 0.05 (3H, s, -OTBDMS), 0.04 (3H, s, -OTBDMS), 0.02 (3H, s, -OTBDMS), 0.01 (3H, s, OTBDMS).

${ }^{13}$ C NMR (125 MHz, $\left.\mathrm{CDCl}_{3}\right): \delta 173.3,171.2,135.9$ (2C), $135.8(2 \mathrm{C}), 133.7,133.3,132.1$, 129.8, 129.7, 127.7 (2C), 127.6 (2C), 118.4, 74.80, 74.75, 68.8, 66.8, 66.3, 65.2, 45.5, 43.8, 43.7, $42.6,42.5,31.8,31.2,26.9$ (3C), 25.9 (3C), 25.8 (3C), 23.3, 19.3, 17.93, 17.88, -4.36 (2C), -4.45, -4.7 .

HRMS (ESI+): Calculated for $\mathrm{C}_{46} \mathrm{H}_{76} \mathrm{O}_{8} \mathrm{NaSi}_{3}[\mathrm{M}+\mathrm{Na}]$ : 863.4746. Found: 863.4748.

$[\alpha]_{D}^{24}=-2.52^{\circ}\left(c=1.46, \mathrm{CHCl}_{3}\right)$. 

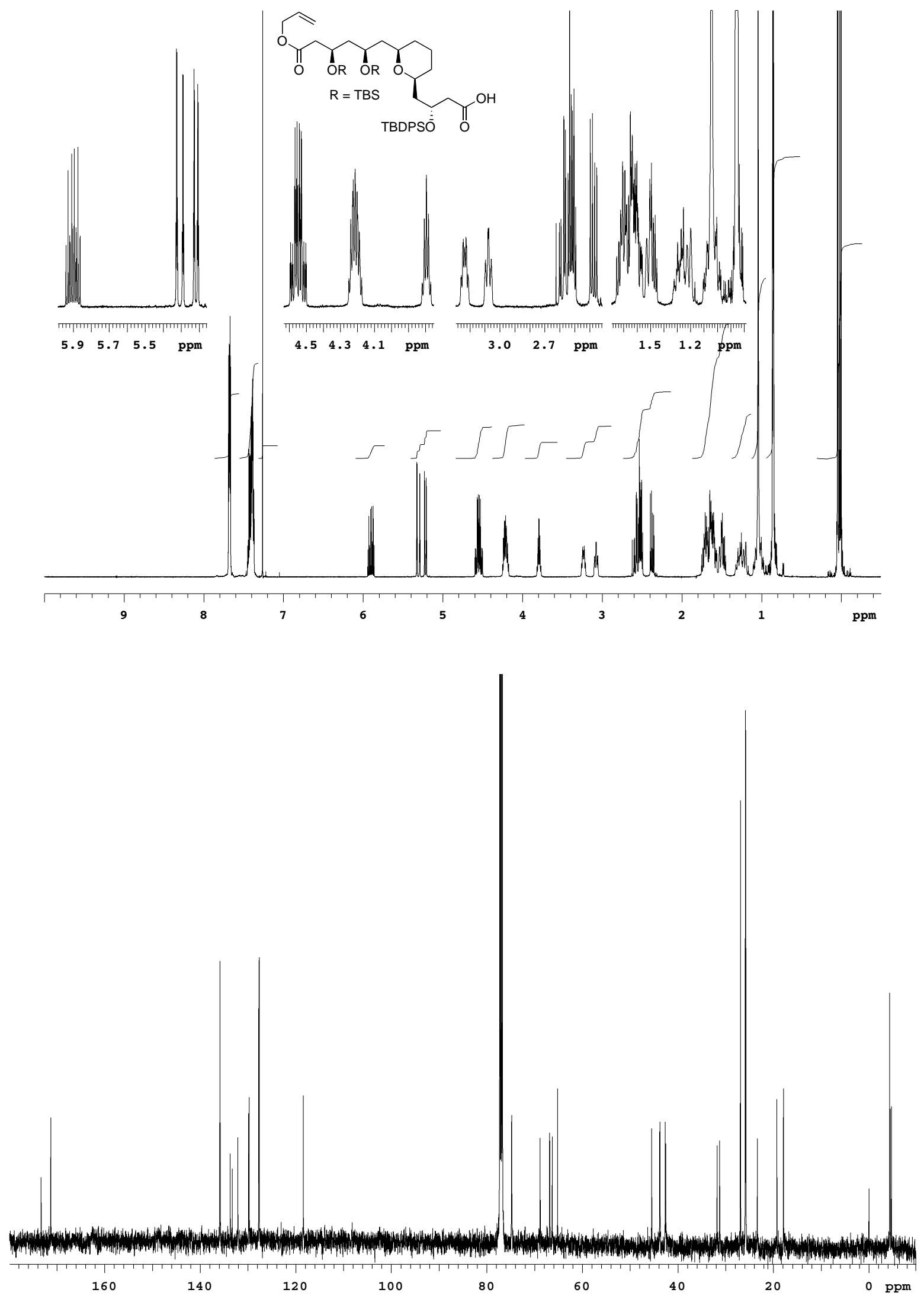


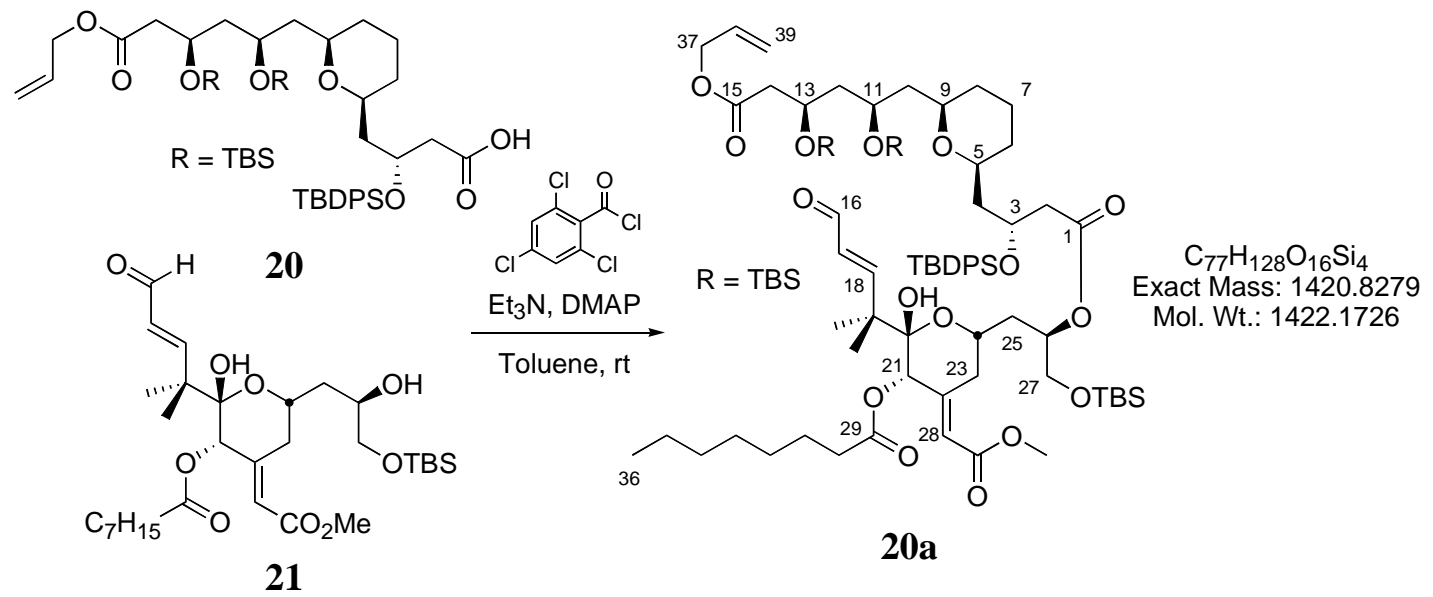

To a solution of carboxylic acid $20(14.0 \mathrm{mg}, 17 \mu \mathrm{mol})$ in toluene $(0.66 \mathrm{~mL})$, under $\mathrm{N}_{2}$ at $\mathrm{rt}$, was added a single portion of triethylamine $(9.7 \mu \mathrm{L}, 70 \mu \mathrm{mol})$ via syringe followed by a single portion of 2,4,6-trichlorobenzoyl chloride $(0.10 \mathrm{~mL}$ of a $170 \mathrm{mM}$ solution in toluene, $17 \mu \mathrm{mol})$ via syringe. The solution was stirred at $\mathrm{rt}$ for $3 \mathrm{~h}$ and then a solution of alcohol 21 (10.9 $\mathrm{mg}, 18.3$ $\mu \mathrm{mol})$ and DMAP $(10 \mathrm{mg}, 83 \mu \mathrm{mol})$ in toluene $(0.76 \mathrm{~mL})$ was added in one portion via cannula. After stirring for $1 \mathrm{~h}$, the solvent was removed under stream of $\mathrm{N}_{2}$ and the residue was directly chromatographed on silica (10\% EtOAc / pentane) to give $16.9 \mathrm{mg}$ of the coupled ester 20a as a colorless oil (72\%).

Data for 20a:

$\mathbf{R}_{f}=0.60$ (15\% EtOAc / petroleum ether) - one black spot w/ p-anisaldehyde stain.

IR (thin film): 3489, 3072, 3049, 2953, 2930, 2857, 2719, 1738, 1725, 1691, 1472, 1463, 1428, 1384, 1361, 1306, 1257, 1229, 1155, 1111, 1083, 1048, 1006, 985, 939, 837, 776, 741, $703 \mathrm{~cm}^{-1}$.

${ }^{1} \mathbf{H}$ NMR $\left(600 \mathrm{MHz}, \mathrm{CDCl}_{3}\right): \delta 9.56(1 \mathrm{H}, \mathrm{d}, J=7.8 \mathrm{~Hz}, \mathrm{C} 16), 7.66(4 \mathrm{H}, \mathrm{m}, \mathrm{Ar}-\mathrm{H}), 7.38(7 \mathrm{H}, \mathrm{m}$, Ar-H \& C18), $5.99(1 \mathrm{H}, \mathrm{d}, J=2.1 \mathrm{~Hz}, \mathrm{C} 28), 5.95(1 \mathrm{H}, \mathrm{dd}, J=7.8,16.1 \mathrm{~Hz}, \mathrm{C} 17), 5.91(1 \mathrm{H}, \mathrm{ddt}$, $J=10.4,17.2,5.9 \mathrm{~Hz}, \mathrm{C} 38), 5.31(1 \mathrm{H}, \mathrm{dq}, J=17.2,1.6 \mathrm{~Hz}, \mathrm{C} 39), 5.22(1 \mathrm{H}, \mathrm{dq}, J=10.4,1.4 \mathrm{~Hz}$, C39), $5.16(1 \mathrm{H}, \mathrm{ddt}, J=1.5,10.6,5.3 \mathrm{~Hz}, \mathrm{C} 26), 5.11(1 \mathrm{H}, \mathrm{s}, \mathrm{C} 21), 4.58(1 \mathrm{H}, \mathrm{ddt}, J=5.8,13.3$, $1.4 \mathrm{~Hz}, \mathrm{C} 37), 4.54(1 \mathrm{H}, \mathrm{ddt}, J=5.9,13.3,1.3 \mathrm{~Hz}, \mathrm{C} 37), 4.29(1 \mathrm{H}, \mathrm{dq}, J=6.9,5.9 \mathrm{~Hz}, \mathrm{C} 3), 4.17$ $(1 \mathrm{H}, \mathrm{dq}, J=5.1,6.6 \mathrm{~Hz}, \mathrm{C} 13), 3.80(2 \mathrm{H}, \mathrm{m}, \mathrm{C} 11 \& \mathrm{C} 24), 3.67(1 \mathrm{H}, \mathrm{m}, \mathrm{C} 23), 3.66(3 \mathrm{H}, \mathrm{s},-$ OMe), $3.64(1 \mathrm{H}, \mathrm{dd}, J=5.0,10.8 \mathrm{~Hz}, \mathrm{C} 27), 3.59(1 \mathrm{H}, \mathrm{dd}, J=5.5,10.8 \mathrm{~Hz}, \mathrm{C} 27), 3.28(1 \mathrm{H}, \mathrm{ddt}$, $J=2.0,5.7,7.4 \mathrm{~Hz}, \mathrm{C} 9), 3.24(1 \mathrm{H}, \mathrm{dddd}, J=2.2,4.2,7.7,10.3 \mathrm{~Hz}, \mathrm{C} 5), 3.16(1 \mathrm{H}, \mathrm{s}, \mathrm{C} 20-\mathrm{OH})$, $2.52(1 \mathrm{H}, \mathrm{dd}, J=5.2,14.7 \mathrm{~Hz}, \mathrm{C} 14), 2.45(1 \mathrm{H}, \mathrm{dd}, J=6.3,15.0 \mathrm{~Hz}, \mathrm{C} 2), 2.41(1 \mathrm{H}, \mathrm{dd}, J=5.2$, $15.0 \mathrm{~Hz}, \mathrm{C} 2), 2.41(1 \mathrm{H}, \mathrm{dd}, J=7.0,14.7 \mathrm{~Hz}, \mathrm{C} 14), 2.09(1 \mathrm{H}, \mathrm{dt}, J=16.0,7.4 \mathrm{~Hz}, \mathrm{C} 30), 2.06$ $(1 \mathrm{H}, \mathrm{m}, \mathrm{C} 23), 2.02(1 \mathrm{H}, \mathrm{dt}, J=16.0,7.6 \mathrm{~Hz}, \mathrm{C} 30), 1.91(1 \mathrm{H}, \mathrm{ddd}, J=1.7,11.4,14.7 \mathrm{~Hz}, \mathrm{C} 25)$, 1.65-1.77 (3H, m, C7, C12 \& C25), 1.52-1.63 (5H, m, C4, C8, C10 \& $\mathrm{C} 12), 1.44-1.52(3 \mathrm{H}, \mathrm{m}$, C10 \& C31), $1.24\left(10 \mathrm{H}, \mathrm{m}, \mathrm{C} 6, \mathrm{C} 7\right.$ \& C32-C35), $1.15\left(6 \mathrm{H}, \mathrm{s},-\mathrm{CH}_{3}\right), 1.01(1 \mathrm{H}, \mathrm{m}, \mathrm{C} 8), 1.00(9 \mathrm{H}$, s, -OTBDMS), 0.94 (1H, m, C6), 0.89 (9H, s, -OTBDMS), 0.87 (3H, m, C36), 0.86 (18H, s, OTBDMS), 0.057 (3H, s, -OTBDMS), 0.055 (3H, s, -OTBDMS), 0.054 (3H, s, -OTBDMS), 0.047 (3H, s, -OTBDMS), 0.03 (3H, s, -OTBDMS), 0.00 (3H, s, -OTBDMS).

${ }^{13} \mathrm{C}$ NMR $\left(125 \mathrm{MHz}, \mathrm{CDCl}_{3}\right): \delta$ 194.6, 172.1, 171.7, 171.0, 166.6, 166.4, 150.5, 135.9 (2C), 135.8 (2C), 133.93, 133.85, 132.1, 129.62, 129.55, 127.58 (2C), 127.56 (2C), 127.4, 120.6, $118.4,99.5,74.6,74.2,72.6,71.2,67.7,66.8,66.3,66.2,65.1,64.9,51.2,46.1,45.7,44.5,43.7$, 
43.03, 43.00, 37.5, 34.5, 31.58, 31.56, 31.1, 30.9, 28.88, 28.86, 26.9 (3C), 25.9 (3C), 25.84 (3C), 25.77 (3C), 24.5, 23.4, 22.9, 22.5, 20.0, 19.3, 18.3, 18.0, 17.9, 14.1, -4.29, -4.28, -4.3, -4.6, -5.3 (2C).

HRMS (ESI+): Calculated for $\mathrm{C}_{77} \mathrm{H}_{128} \mathrm{O}_{16} \mathrm{NaSi}_{4}[\mathrm{M}+\mathrm{Na}]$ : 1443.8177 . Found: 1443.8151 . $[\alpha]_{D}^{26}=-24.2^{\circ}\left(c=1.35, \mathrm{CHCl}_{3}\right)$. 

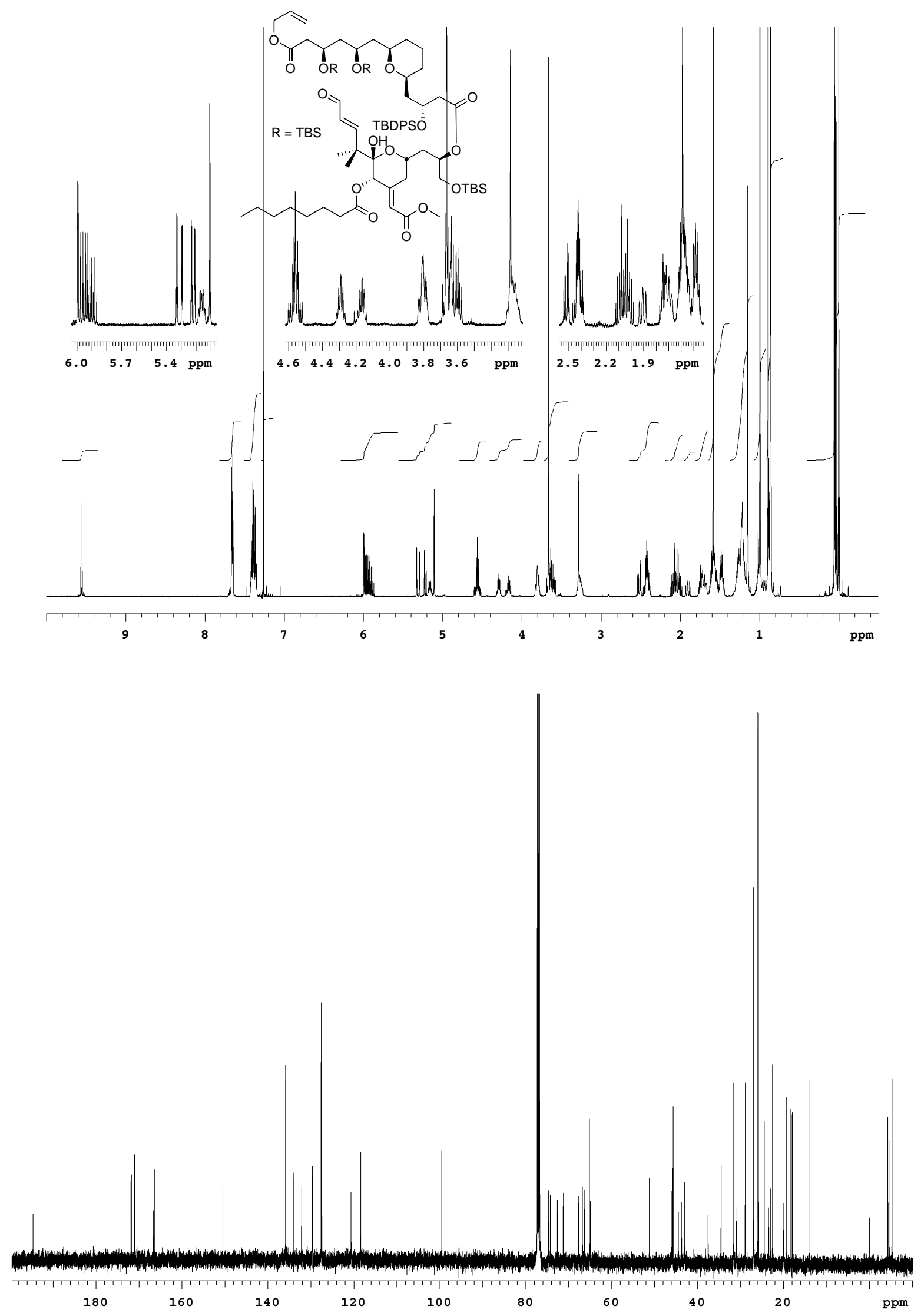


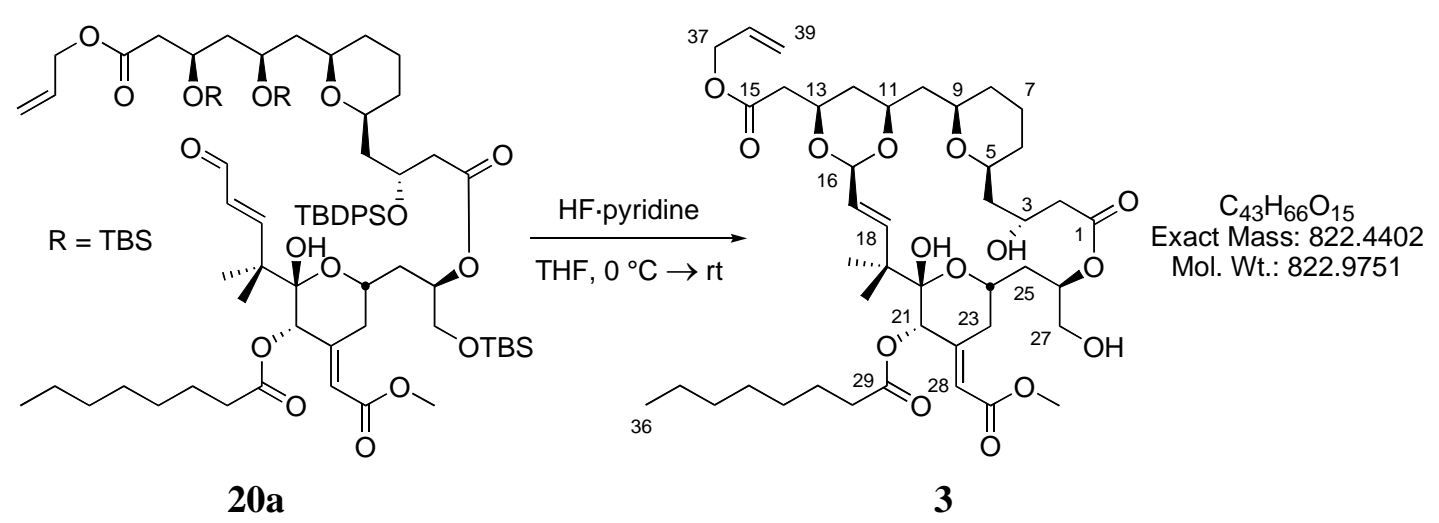

To a solution of the seco aldehyde 20a $(16.3 \mathrm{mg}, 11.5 \mu \mathrm{mol})$ in THF $(3.0 \mathrm{~mL})$, under $\mathrm{N}_{2}$ at 0 ${ }^{\circ} \mathrm{C}$ in a $10 \mathrm{~mL}$ polypropylene vial, was added HF.pyridine $(0.58 \mathrm{~mL})$ dropwise over $2 \mathrm{~min}$. The 0 ${ }^{\circ} \mathrm{C}$ bath was removed and the reaction was allowed to stir for $32 \mathrm{~h}$ at room temperature. The reaction solution was transferred to a larger $50 \mathrm{~mL}$ propylene vial using EtOAc $(10 \mathrm{~mL})$. Water $(4 \mathrm{~mL})$ was added followed by slow addition of sat aq. $\mathrm{NaHCO}_{3}$ until gas evolution ceased (13 $\mathrm{mL}$, vigorous bubbling!). The layers were separated and the aqueous phase was extracted with EtOAc $(3 \times 10 \mathrm{~mL})$. The combined organic layers were dried over anhydrous $\mathrm{Na}_{2} \mathrm{SO}_{4}$, filtered and concentrated in vacuo. Chromatography on silica (70\% EtOAc / pentane) gave $6.8 \mathrm{mg}$ of the completed analog 3 as a white amorphous solid (72\%).

Data for 3:

$\mathbf{R}_{f}=0.43$ (75\% EtOAc / petroleum ether) - one black spot w/ p-anisaldehyde stain.

IR (thin film): $3457,3325,2933,2858,1738,1733,1667,1435,1407,1383,1362,1347,1298$, $1258,1230,1151,1136,1108,1088,1052,1003,983,890,861,756 \mathrm{~cm}^{-1}$.

${ }^{1} \mathbf{H}$ NMR $\left(600 \mathrm{MHz}, \mathrm{CDCl}_{3}\right): \delta 5.98(1 \mathrm{H}, \mathrm{d}, J=2.2 \mathrm{~Hz}, \mathrm{C} 28), 5.96(1 \mathrm{H}, \mathrm{d}, J=15.9 \mathrm{~Hz}, \mathrm{C} 18)$, $5.89(1 \mathrm{H}, \mathrm{ddt}, J=10.6,17.2,5.6 \mathrm{~Hz}, \mathrm{C} 38), 5.38(1 \mathrm{H}, \mathrm{dd}, J=7.6,15.9 \mathrm{~Hz}, \mathrm{C} 17), 5.36(1 \mathrm{H}, \mathrm{m}$, C26), $5.32(1 \mathrm{H}, \mathrm{dq}, J=17.2,1.5 \mathrm{~Hz}, \mathrm{C} 37), 5.22(1 \mathrm{H}, \mathrm{dq}, J=1.6,1.3 \mathrm{~Hz}, \mathrm{C} 37), 5.14(1 \mathrm{H}, \mathrm{s}$, C21), $5.10(1 \mathrm{H}, \mathrm{s}, \mathrm{C} 20-\mathrm{OH}), 5.06(1 \mathrm{H}, \mathrm{d}, J=7.5 \mathrm{~Hz}, \mathrm{C} 16), 4.59(2 \mathrm{H}, \mathrm{dt}, J=5.6,1.5 \mathrm{~Hz}, \mathrm{C} 39)$, $4.47(1 \mathrm{H}, \mathrm{d}, J=11.8, \mathrm{C} 3-\mathrm{OH}), 4.18(2 \mathrm{H}, \mathrm{m}, \mathrm{C} 3 \& \mathrm{C} 13), 4.05(1 \mathrm{H}, \mathrm{tt}, J=2.6,11.4 \mathrm{~Hz}, \mathrm{C} 24)$, 3.92 (1H, ddd, $J=2.7,7.4,11.3 \mathrm{~Hz}, \mathrm{C} 11), 3.86(1 \mathrm{H}, \mathrm{brd}, J=12.1 \mathrm{~Hz}, \mathrm{C} 27), 3.70(1 \mathrm{H}, \mathrm{m}, \mathrm{C} 24)$, $3.68\left(3 \mathrm{H}, \mathrm{s},-\mathrm{OCH}_{3}\right), 3.66(1 \mathrm{H}, \mathrm{m}, \mathrm{C} 27), 3.53(1 \mathrm{H}, \mathrm{m}, \mathrm{C} 5), 3.44(1 \mathrm{H}, \mathrm{m}, \mathrm{C} 9), 2.70(1 \mathrm{H}, \mathrm{dd}, J=$ $6.1,15.5 \mathrm{~Hz}, \mathrm{C} 14), 2.51(2 \mathrm{H}, \mathrm{m}, \mathrm{C} 2), 2.46(1 \mathrm{H}, \mathrm{dd}, J=7.5,15.5 \mathrm{~Hz}, \mathrm{C} 14), 2.29$ (2H, m, C30), $2.01(4 \mathrm{H}, \mathrm{m}, \mathrm{C} 4, \mathrm{C} 24, \mathrm{C} 25$ \& $\mathrm{C} 27-\mathrm{OH}), 1.80(3 \mathrm{H}, \mathrm{m}, \mathrm{C} 7, \mathrm{C} 10$ \& $\mathrm{C} 25), 1.35-1.63(9 \mathrm{H}, \mathrm{m}, \mathrm{C} 4$, $\mathrm{C} 6, \mathrm{C} 7, \mathrm{C} 8, \mathrm{C} 10, \mathrm{C} 12$ \& $\mathrm{C} 31), 1.26(10 \mathrm{H}, \mathrm{m}, \mathrm{C} 6, \mathrm{C} 8$ \& C32-C35), $1.17(3 \mathrm{H}, \mathrm{s},-\mathrm{CH}), 1.02(3 \mathrm{H}$, $\left.\mathrm{s},-\mathrm{CH}_{3}\right), 0.87(3 \mathrm{H}, \mathrm{t}, J=7.1 \mathrm{~Hz}, \mathrm{C} 36)$.

${ }^{13} \mathrm{C}$ NMR $\left(100 \mathrm{MHz}, \mathrm{CDCl}_{3}\right): \delta 172.5,172.1,170.2,167.0,151.7,142.7,131.9,125.3,119.8$, 118.2, 102.4, 98.9, 78.6, 75.8, 75.4, 74.1, 72.3, 71.6, 68.6, 65.8, 65.2, 64.4, 51.1, 45.1, 42.8, 42.5, 40.9, 39.9, 37.7, 35.9, 34.6, 31.6, 31.4, 31.3, 31.0, 29.0, 28.9, 24.7, 24.3, 23.0, 22.6, 19.3, 14.1 .

HRMS (ESI+): Calculated for $\mathrm{C}_{43} \mathrm{H}_{66} \mathrm{O}_{15} \mathrm{Na}[\mathrm{M}+\mathrm{Na}]:$ 845.4299. Found: 845.4321.

$[\alpha]_{D}^{23}=-5.7^{\circ}\left(c=0.68, \mathrm{CHCl}_{3}\right)$. 

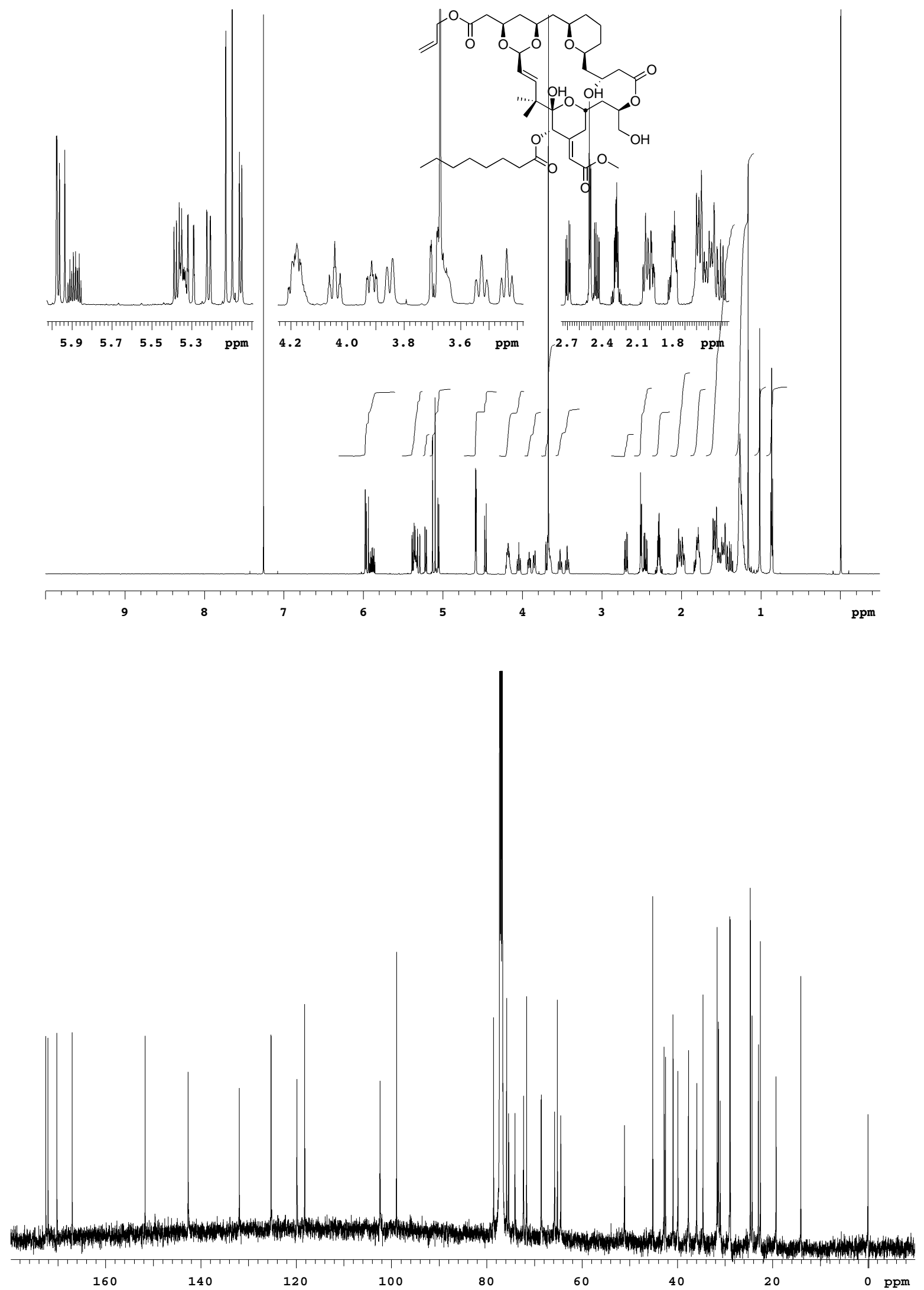

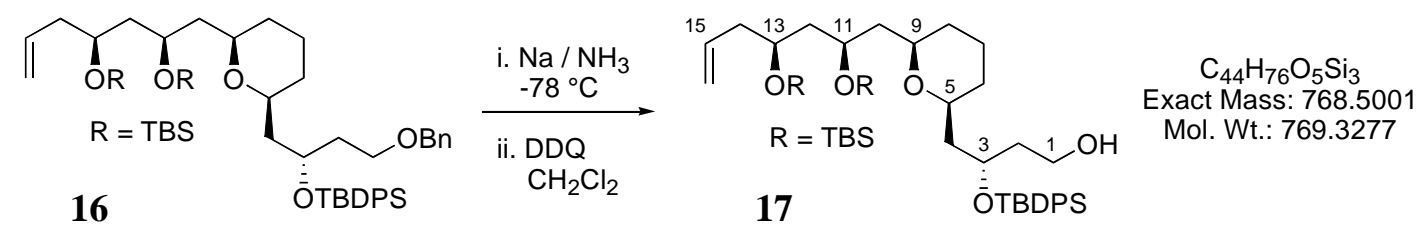

Into a flask containing benzyl ether 16 under $\mathrm{N}_{2}$ at $-78{ }^{\circ} \mathrm{C}$ was condensed $\mathrm{NH}_{3}$ (ca. $15 \mathrm{~mL}$ ). To this solution was added a single piece of sodium metal $(10 \mathrm{mg}, 0.43 \mathrm{mmol})$. The deep blue colored solution was allowed to stir for $2 \mathrm{~h}$ at $-78^{\circ} \mathrm{C}$. After portionwise addition of solid $\mathrm{NH}_{4} \mathrm{Cl}$ $(2 \mathrm{~g})$, the cold bath was removed and the solvent was allowed to evaporate as the flask warmed to $\mathrm{rt}$. The residue was transferred to a separatory funnel containing water $(30 \mathrm{~mL}) \mathrm{using} \mathrm{Et}_{2} \mathrm{O}(20$ $\mathrm{mL})$. The layers were separated and the aqueous layer was further extracted with $\mathrm{Et}_{2} \mathrm{O}(2 \times 20$ $\mathrm{mL}$ ). The combined organic layers were dried over anhydrous $\mathrm{MgSO}_{4}$, filtered and concentrated in vacuo to give a yellow liquid.

To a solution of the crude liquid in $\mathrm{CH}_{2} \mathrm{Cl}_{2}(1.0 \mathrm{~mL})$ was added a single portion of solid DDQ (20 mg, $0.088 \mathrm{mmol})$. The orange suspension was allowed to stir for $1 \mathrm{~h} \mathrm{at} \mathrm{rt}$ and then diluted with water $(1 \mathrm{~mL})$ and sat. aq. $\mathrm{Na}_{2} \mathrm{~S}_{2} \mathrm{O}_{3}(1.5 \mathrm{~mL})$. The reaction mixture was extracted with $\mathrm{Et}_{2} \mathrm{O}(3 \times 3 \mathrm{~mL})$ and the combined organic layers were dried over anhydrous $\mathrm{MgSO}_{4}$, filtered and concentrated in vacuo. Chromatography on silica $(5 \rightarrow 20 \%$ EtOAc / pentane) gave $3.7 \mathrm{mg}$ of alcohol 17 as a pale yellow oil (21\%). Even though the isolated material was a single spot by TLC analysis, the ${ }^{1} \mathrm{H}-\mathrm{NMR}$ spectrum shows a small amount of impurity that could not be separated from the product.

\section{Data for 16b:}

$\mathbf{R}_{f}=0.34$ (10\% EtOAc / hexanes ether) — one black spot w/ p-anisaldehyde stain.

IR (thin film): 3452 (br), 3072, 1929, 2857, 1472, 1463, 1428, 1389, 1361, 1256, 1198, 1105, $1067,1046,1005,938,913,837,774,739,702 \mathrm{~cm}^{-1}$.

${ }^{1} \mathbf{H}$ NMR (500 MHz, $\left.\mathrm{CDCl}_{3}\right): \delta 7.69(4 \mathrm{H}, \mathrm{m}, \mathrm{ArH}), 7.41(6 \mathrm{H}, \mathrm{m}, \mathrm{ArH}), 5.78(1 \mathrm{H}, \mathrm{ddt}, J=10.4$, 17.4, 7.1 Hz, C15), 5.03 (2H, m, C16), 4.05 (1H, dq, J =6.8, 5.0 Hz, C3), 3.79 (3H, m, C1, C11 \& C13), $3.72(1 \mathrm{H}, \mathrm{m}, \mathrm{C} 1), 3.22(1 \mathrm{H}, \mathrm{ddt}, J=1.8,11.0,6.6 \mathrm{~Hz}, \mathrm{C} 9), 2.96(1 \mathrm{H}$, dddd, $J=2.2,3.5$, 8.2, $10.8 \mathrm{~Hz}, \mathrm{C} 5), 2.39$ (1H, dd, $J=4.6,6.5 \mathrm{~Hz}, \mathrm{C} 1-\mathrm{OH}), 2.23$ (1H, dddt, $J=5.5,6.8,13.7,1.3$ $\mathrm{Hz}, \mathrm{C} 14), 2.10$ (1H, dddt, $J=5.5,6.7,13.9,1.1 \mathrm{~Hz}, \mathrm{C} 14), 1.86(1 \mathrm{H}, \mathrm{ddt}, J=8.0,14.3,4.9 \mathrm{~Hz}$, C2), 1.64-1.79 (4H, m, C2, C4, C7 \& C12), 1.47-1.62 (4H, m, C4, C8, C10 \& C12), 1.43 (1H, dt, $J=13.5,6.3 \mathrm{~Hz}, \mathrm{C} 10), 1.24(1 \mathrm{H}, \mathrm{qt}, J=13.1,4.0 \mathrm{~Hz}, \mathrm{C} 7), 1.16(1 \mathrm{H}, \mathrm{brd}, J=13.8 \mathrm{~Hz}, \mathrm{C} 6), 1.05$ $(9 \mathrm{H}, \mathrm{s}, t-\mathrm{Bu}), 0.97(2 \mathrm{H}, \mathrm{m}, \mathrm{C} 6$ \& $\mathrm{C} 8), 0.88(9 \mathrm{H}, \mathrm{s}, t-\mathrm{Bu}), 0.86(9 \mathrm{H}, \mathrm{s}, t-\mathrm{Bu}), 0.037(3 \mathrm{H}, \mathrm{s},-$ OTBDMS), 0.035 (3H, s, -OTBDMS), 0.02 (3H, s, -OTBDMS), 0.004 (3H, s, -OTBDMS).

${ }^{13} \mathrm{C}$ NMR (125 MHz, $\left.\mathrm{CDCl}_{3}\right): \delta 135.90$ (2C), 135.86 (2C), 134.9, 134.0, 133.8, 129.7, 129.6, 127.7 (2C), 127.6 (2C), 117.0, 75.2, 74.7, 71.0, 68.9, 66.5, 59.5, 45.2, 43.9, 43.6, 41.9, 38.8, $31.8,31.2,27.0$ (3C), 25.9 (6C), 23.4, 19.3, 18.02, 17.96, -4.2, -4.3, -4.4 (2C).

HRMS (ESI+): Calculated for $\mathrm{C}_{44} \mathrm{H}_{76} \mathrm{O}_{5} \mathrm{NaSi}_{3}[\mathrm{M}+\mathrm{Na}]:$ 791.4898. Found: 791.4896.

$$
[\alpha]_{D}^{27}=-7.20^{\circ}\left(c=0.32, \mathrm{CHCl}_{3}\right) \text {. }
$$



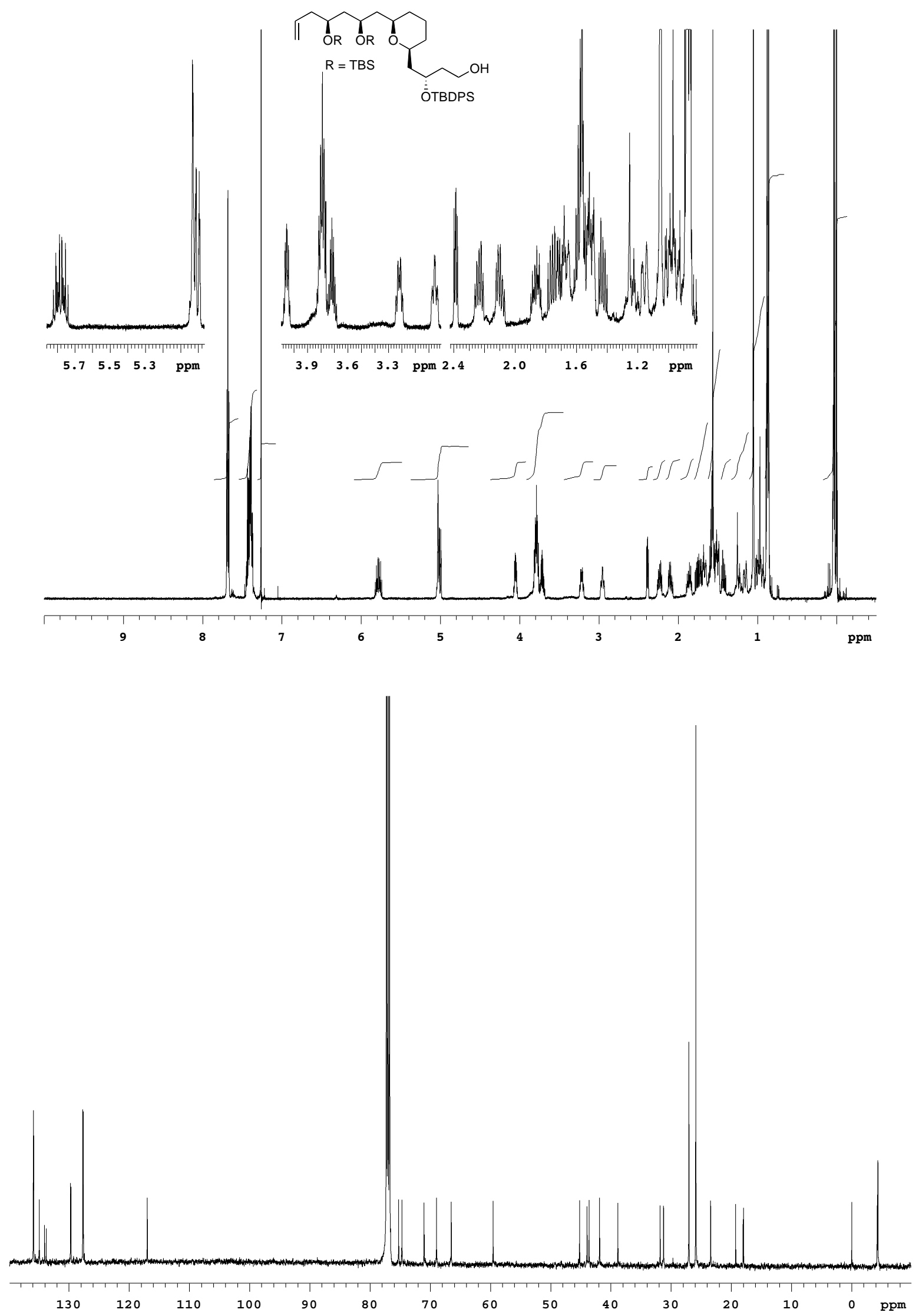


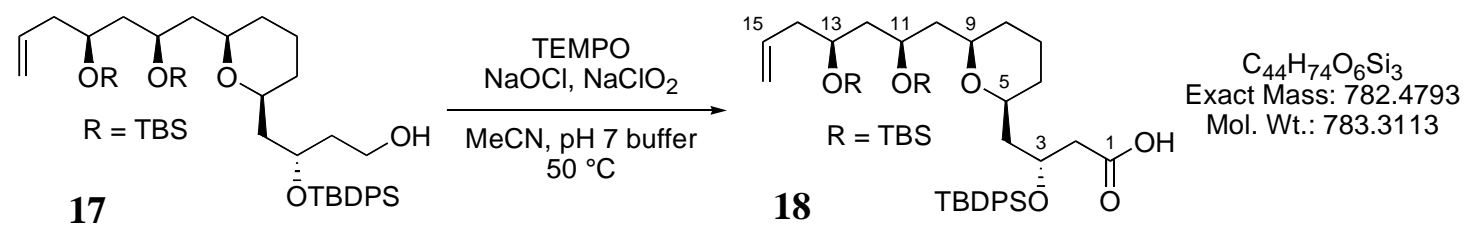

To a solution of alcohol $17(3.3 \mathrm{mg}, 0.0043 \mathrm{mmol})$ in a mixture of $\mathrm{pH} 7$ phosphate buffer $(0.25 \mathrm{~mL})$ and $\mathrm{MeCN}(0.35 \mathrm{~mL})$, was added a single portion of solid TEMPO (0.5 mg, 0.0032 mmol), a single portion of solid $\mathrm{NaClO}_{2}(80 \%$ technical grade, $11 \mathrm{mg}, 0.097 \mathrm{mmol})$ and a single portion of $\mathrm{NaOCl}$ (as a $5 \%$ commercial bleach solution, $3 \mu \mathrm{L}$ ) via syringe. Upon addition of the $\mathrm{NaOCl}$ solution, the reaction turned a deep purple color. The reaction was allowed to stir for $1 \mathrm{~h}$ at $50{ }^{\circ} \mathrm{C}$ and then diluted with brine $(2 \mathrm{~mL})$ and extracted with $\mathrm{Et}_{2} \mathrm{O}(4 \times 2 \mathrm{~mL})$. The combined organic layers were dried over anhydrous $\mathrm{MgSO}_{4}$, filtered and concentrated. Chromatography on silica $(20 \%$ EtOAc / pentane $+0.5 \% \mathrm{AcOH})$ gave $3.2 \mathrm{mg}$ of carboxylic acid $\mathbf{1 8}$ as a colorless oil $(95 \%)$.

Data for 17:

$\mathbf{R}_{f}=0.43$ (20\% EtOAc / petroleum ether) — one black spot w/ p-anisaldehyde stain.

IR (thin film): 3072, 2930, 2857, 1712, 1590, 1472, 1463, 1428, 1389, 1361, 1307, 1111, 1082, $1005,966,938,914,836,807,774,739,702 \mathrm{~cm}^{-1}$.

${ }^{1} \mathbf{H}$ NMR $\left(500 \mathrm{MHz}, \mathrm{CDCl}_{3}\right): \delta 7.67(4 \mathrm{H}, \mathrm{m}, \operatorname{ArH}), 7.41(6 \mathrm{H}, \mathrm{m}, \operatorname{ArH}), 5.77(1 \mathrm{H}, \mathrm{ddt}, J=10.8$, 16.5, 7.2 Hz, C15), $5.02(2 \mathrm{H}, \mathrm{m}, \mathrm{C} 16), 4.21(1 \mathrm{H}, \mathrm{dq}, J=7.1,5.6 \mathrm{~Hz}, \mathrm{C} 3), 3.80(1 \mathrm{H}$, pent, $J=6.6$ $\mathrm{Hz}, \mathrm{C} 11), 3.75$ (1H, ddt, $J=6.8,11.4,5.7 \mathrm{~Hz}, \mathrm{C} 13), 3.29(1 \mathrm{H}$, dddd, $J=1.5,5.3,7.1,11.0 \mathrm{~Hz}$, C9), 3.10 (1H, ddt, $J=9.2,10.9,2.2 \mathrm{~Hz}, \mathrm{C} 5), 2.57(1 \mathrm{H}, \mathrm{dd}, J=5.6,14.7 \mathrm{~Hz}, \mathrm{C} 2), 2.51$ (1H, dd, $J$ $=4.9,14.7 \mathrm{~Hz}, \mathrm{C} 2), 2.23(1 \mathrm{H}, \mathrm{dddt}, J=5.8,6.9,13.7,1.1 \mathrm{~Hz}, \mathrm{C} 14), 2.11(1 \mathrm{H}$, dddt, $J=5.3,7.2$, 13.7, $1.0 \mathrm{~Hz}, \mathrm{C} 14), 1.73$ (1H, ddd, $J=7.3,9.0,14.6 \mathrm{~Hz}, \mathrm{C} 4), 1.71$ (1H, brd, $J=13.0 \mathrm{~Hz}, \mathrm{C} 7)$, $1.58(5 \mathrm{H}, \mathrm{m}, \mathrm{C} 4, \mathrm{C} 8, \mathrm{C} 10$ \& C12), $1.48(1 \mathrm{H}, \mathrm{ddd}, J=5.5,7.4,13.1 \mathrm{~Hz}, \mathrm{C} 10), 1.28(1 \mathrm{H}, \mathrm{qt}, J=$ 13.1, $3.6 \mathrm{~Hz}, \mathrm{C} 7), 1.23(1 \mathrm{H}$, brd, $J=13.8 \mathrm{~Hz}, \mathrm{C} 6), 1.05$ (2H, m, C6 \& C8), 1.05 (9H, s, $t-\mathrm{Bu})$, $0.87(9 \mathrm{H}, \mathrm{s}, t-\mathrm{Bu}), 0.85$ (9H, s, t-Bu), 0.039 (3H, s, -OTBDMS), 0.037 (3H, s, -OTBDMS), 0.02 (3H, s, -OTBDMS), 0.002(3H, s, -OTBDMS).

${ }^{13} \mathrm{C}$ NMR (125 MHz, $\left.\mathrm{CDCl}_{3}\right): \delta 172.6,135.9$ (2C), $135.8(2 \mathrm{C}), 134.7,133.6,133.2,129.9$, 129.8, 127.73 (2C), 127.66 (2C), 117.1, 75.0, 74.7, 69.0, 68.7, 66.4, 45.4, 43.6 (2C), 42.4, 41.9, 31.8, 30.9, 26.9 (3C), 25.88 (3C), 25.87 (3C), 23.3, 19.2, 18.01, 17.97, -4.18, -4.25, -4.40, -4.42.

HRMS (ESI+): Calculated for $\mathrm{C}_{44} \mathrm{H}_{74} \mathrm{O}_{6} \mathrm{NaSi}_{3}[\mathrm{M}+\mathrm{Na}]$ : 805.4691. Found: 805.4678.

$[\alpha]_{D}^{23}=-6.05^{\circ}\left(c=0.53, \mathrm{C}_{6} \mathrm{D}_{6}\right)$. 

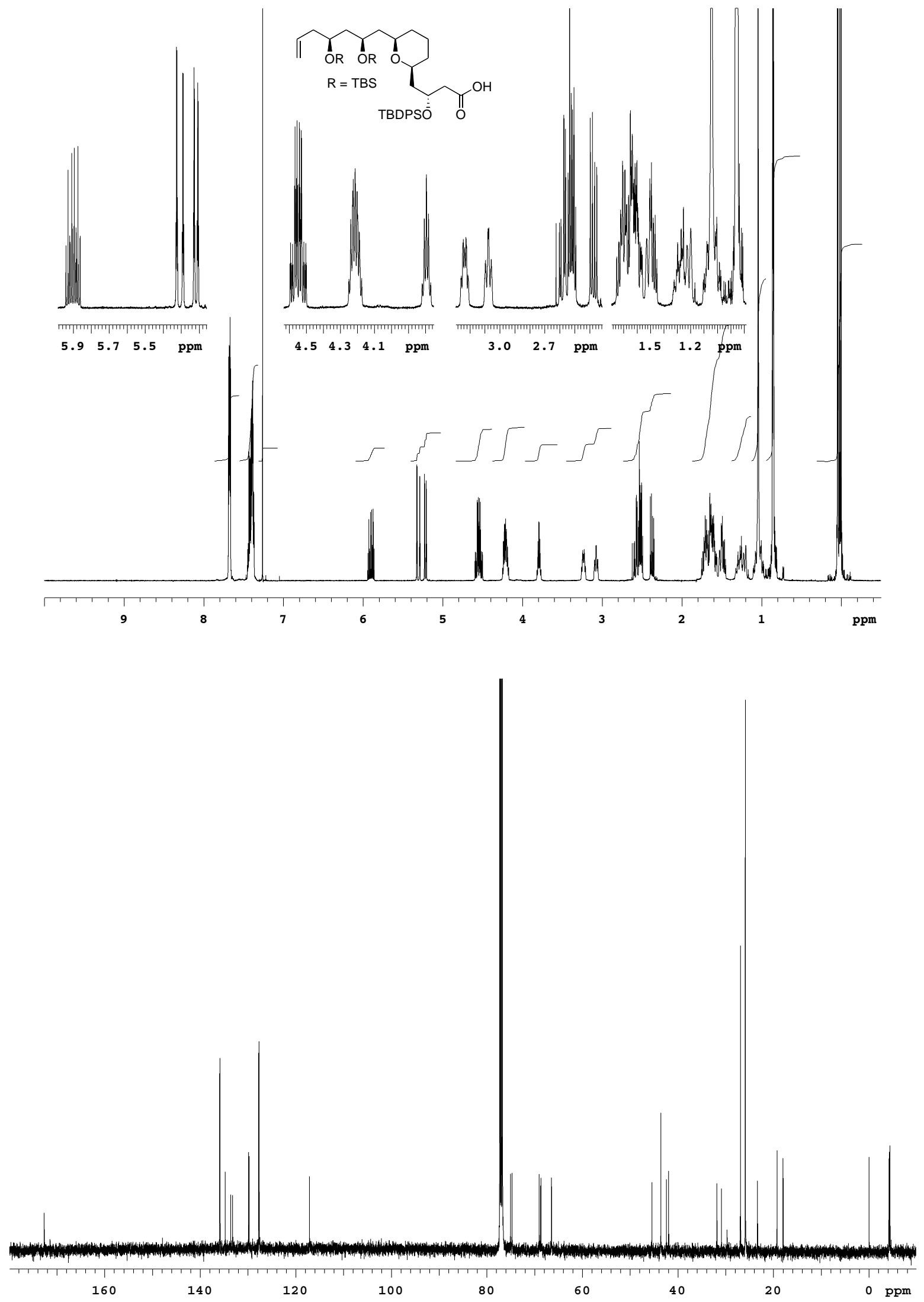


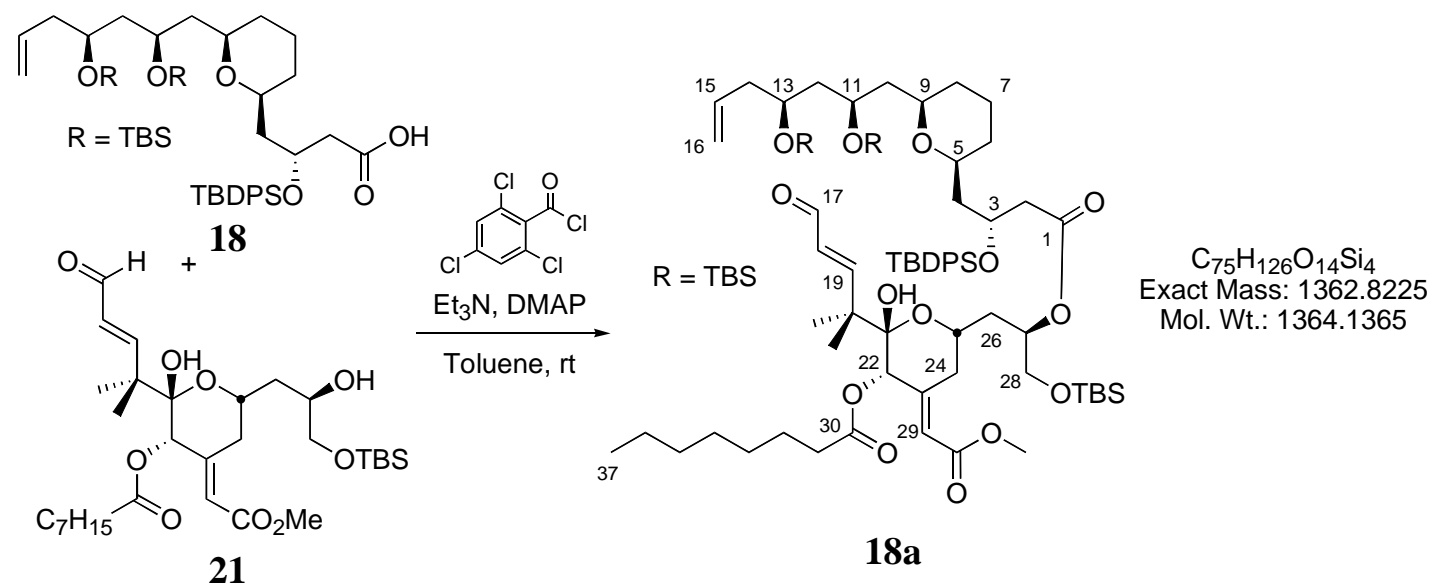

To a solution of carboxylic acid $18(5.1 \mathrm{mg}, 6.5 \mu \mathrm{mol})$ in toluene $(0.13 \mathrm{~mL})$, under $\mathrm{N}_{2}$ at $\mathrm{rt}$, was added a single portion of triethylamine $(3.8 \mu \mathrm{L}, 27 \mu \mathrm{mol})$ via syringe followed by a single portion of 2,4,6-trichlorobenzoyl chloride $(0.20 \mathrm{~mL}$ of a $33 \mathrm{mM}$ solution in toluene, $6.5 \mu \mathrm{mol})$ via syringe. The solution was stirred at $\mathrm{rt}$ for $3 \mathrm{~h}$ and then a solution of alcohol 21 (4.3 mg, 7.2 $\mu \mathrm{mol})$ and DMAP $(4.0 \mathrm{mg}, 33 \mu \mathrm{mol})$ in toluene $(0.33 \mathrm{~mL})$ was added in one portion via cannula. After stirring for $1 \mathrm{~h}$, the solvent was removed under stream of $\mathrm{N}_{2}$ and the residue was directly chromatographed on silica (10\% EtOAc / pentane) to give $5.8 \mathrm{mg}$ of the coupled ester 18a as a colorless oil $(65 \%)$.

Data for 18a:

$\mathbf{R}_{f}=0.39$ (10\% EtOAc / petroleum ether) 一 one black spot w/ p-anisaldehyde stain.

IR (thin film): 3489, 3072, 2953, 2930, 2857, 2714, 1725, 1691, 1472, 1429, 1389, 1361, 1302, $1257,1228,1155,1111,1084,1046,1005,983,938,915,837,775,741,702 \mathrm{~cm}^{-1}$.

${ }^{1} \mathbf{H}$ NMR $\left(500 \mathrm{MHz}, \mathrm{CDCl}_{3}\right): \delta 9.56(1 \mathrm{H}, \mathrm{d}, J=7.8 \mathrm{~Hz}, \mathrm{C} 17), 7.65(4 \mathrm{H}, \mathrm{m}, \mathrm{Ar}-\mathbf{H}), 7.39(7 \mathrm{H}, \mathrm{m}$, Ar-H \& C19), $5.99(1 \mathrm{H}, \mathrm{d}, J=1.9 \mathrm{~Hz}, \mathrm{C} 29), 5.94(1 \mathrm{H}, \mathrm{dd}, J=7.8,16.1 \mathrm{~Hz}, \mathrm{C} 18), 5.78$ (1H, ddt, $J=10.4,17.6,7.0 \mathrm{~Hz}, \mathrm{C} 15), 5.16(1 \mathrm{H}, \mathrm{ddt}, J=1.5,10.8,5.4 \mathrm{~Hz}, \mathrm{C} 27), 5.10(1 \mathrm{H}, \mathrm{s}, \mathrm{C} 22), 5.04$ $(2 \mathrm{H}, \mathrm{m}, \mathrm{C} 16), 4.29(1 \mathrm{H}$, pent, $J=5.9 \mathrm{~Hz}, \mathrm{C} 3), 3.80(2 \mathrm{H}, \mathrm{m}, \mathrm{C} 11 \& \mathrm{C} 25), 3.72(1 \mathrm{H}, \mathrm{m}, \mathrm{C} 13)$, $3.67(1 \mathrm{H}, \mathrm{m}, \mathrm{C} 24), 3.66(3 \mathrm{H}, \mathrm{s},-\mathrm{OMe}), 3.63(1 \mathrm{H}, \mathrm{dd}, J=5.2,10.8 \mathrm{~Hz}, \mathrm{C} 28), 3.58(1 \mathrm{H}, \mathrm{dd}, J=$ 5.4, $10.8 \mathrm{~Hz}, \mathrm{C} 28), 3.31(2 \mathrm{H}, \mathrm{m}, \mathrm{C} 5 \& \mathrm{C} 9), 3.20(1 \mathrm{H}, \mathrm{s}, \mathrm{C} 21-\mathrm{OH}), 2.44(1 \mathrm{H}, \mathrm{dd}, J=6.4,15.0$ $\mathrm{Hz}, \mathrm{C} 2), 2.39(1 \mathrm{H}, \mathrm{dd}, J=6.2,15.0 \mathrm{~Hz}, \mathrm{C} 2), 2.24(1 \mathrm{H}, \mathrm{m}, \mathrm{C} 14), 1.97-2.18(4 \mathrm{H}, \mathrm{m}, \mathrm{C} 14, \mathrm{C} 24$ \& C31), 1.90 (1H, ddd, $J=1.3,11.2,14.4 \mathrm{~Hz}, \mathrm{C} 26), 1.62-1.75$ (3H, m, C7, C12 \& C26), 1.41-1.60 $(8 \mathrm{H}, \mathrm{m}, \mathrm{C} 4, \mathrm{C} 8, \mathrm{C} 10, \mathrm{C} 12, \mathrm{C} 32), 1.23(10 \mathrm{H}, \mathrm{m}, \mathrm{C} 6, \mathrm{C} 7, \mathrm{C} 33-\mathrm{C} 36), 1.14(6 \mathrm{H}, \mathrm{s},-\mathrm{CH}), 0.99(9 \mathrm{H}$, s, -OTBDMS), 0.98 (2H, m, C6 \& C8), 0.89 (9H, s, -OTBDMS), 0.88 (9H, s, -OTBDMS), 0.87 ( $3 \mathrm{H}, \mathrm{m}, \mathrm{C} 37), 0.86$ (9H, s, -OTBDMS), 0.05 (3H, s, -OTBDMS), 0.050 (3H, s, -OTBDMS), 0.048 (3H, s, -OTBDMS), 0.043 (3H, s, -OTBDMS), 0.033 (3H, s, -OTBDMS), -0.005 (3H, s, OTBDMS).

${ }^{13}$ C NMR $\left(125 \mathrm{MHz}, \mathrm{CDCl}_{3}\right): \delta 194.6,172.1,171.7,166.6,166.4,150.5,135.9$ (2C), 135.8 (2C), 134.7, 133.89, 133.86, 129.6, 129.5, 127.60 (2C), 127.57 (2C), 127.4, 120.6, 117.1, 99.5, $74.8,74.1,72.6,71.1,69.0,67.5,66.5,66.2,64.9,51.2,45.74,45.66,44.51,43.5,43.0,42.3$, $37.5,34.5,31.65,31.58,30.91,30.86,28.88,28.86,27.0$ (3C), 25.92 (3C), 25.87 (3C), 25.83 (3C), 24.5, 23.4, 22.9, 22.5, 20.0, 19.3, 18.2, 17.994, 17.986, 14.1, -4.11, -4.14, -4.3, -4.4, -5.286, -5.294 . 
HRMS (ESI+): Calculated for $\mathrm{C}_{75} \mathrm{H}_{126} \mathrm{O}_{14} \mathrm{NaSi}_{4}[\mathrm{M}+\mathrm{Na}]$ : 1385.8122. Found: 1385.8168 .

$[\alpha]_{D}^{23}=-15.5^{\circ}\left(c=0.58, \mathrm{CHCl}_{3}\right)$. 

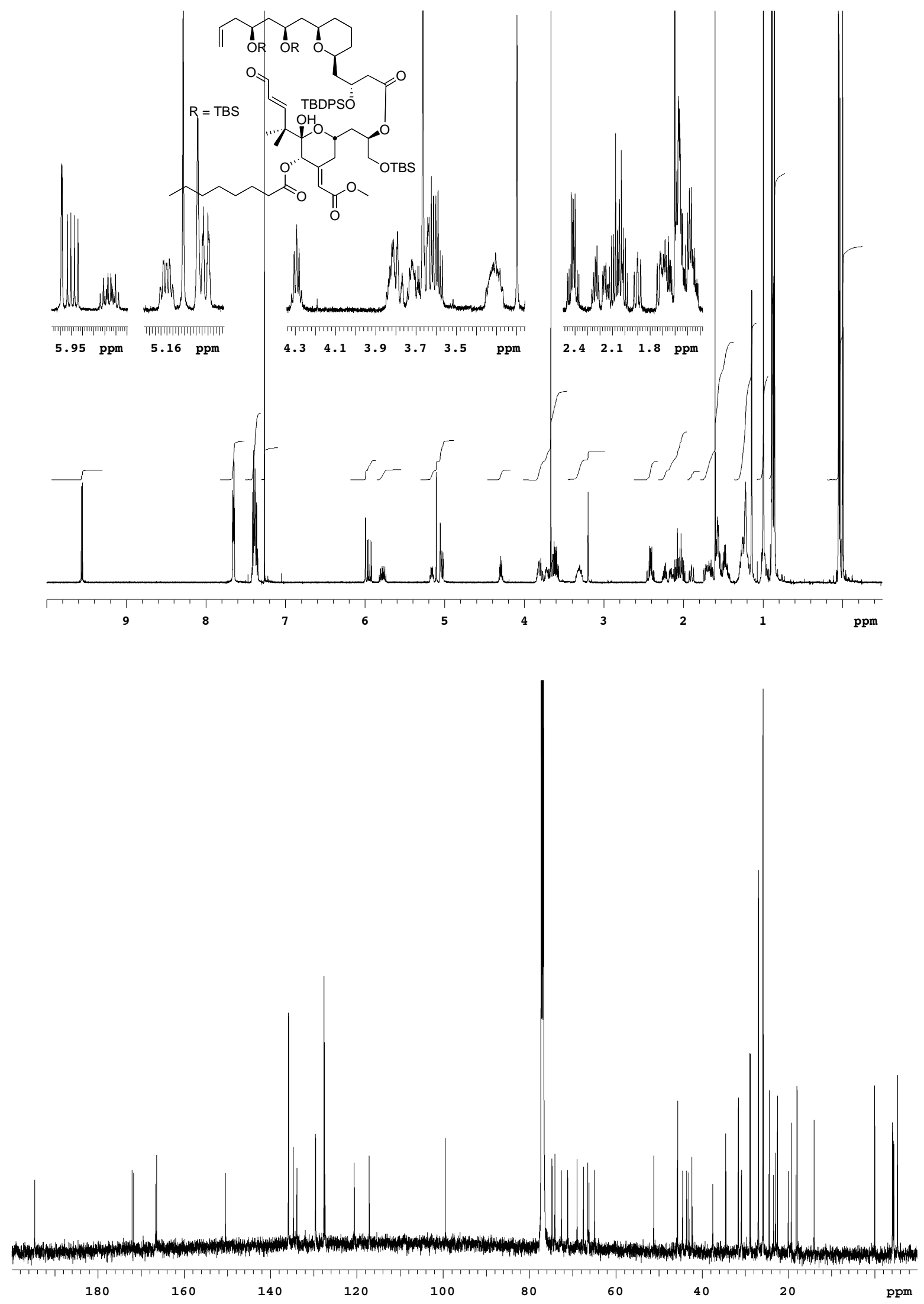


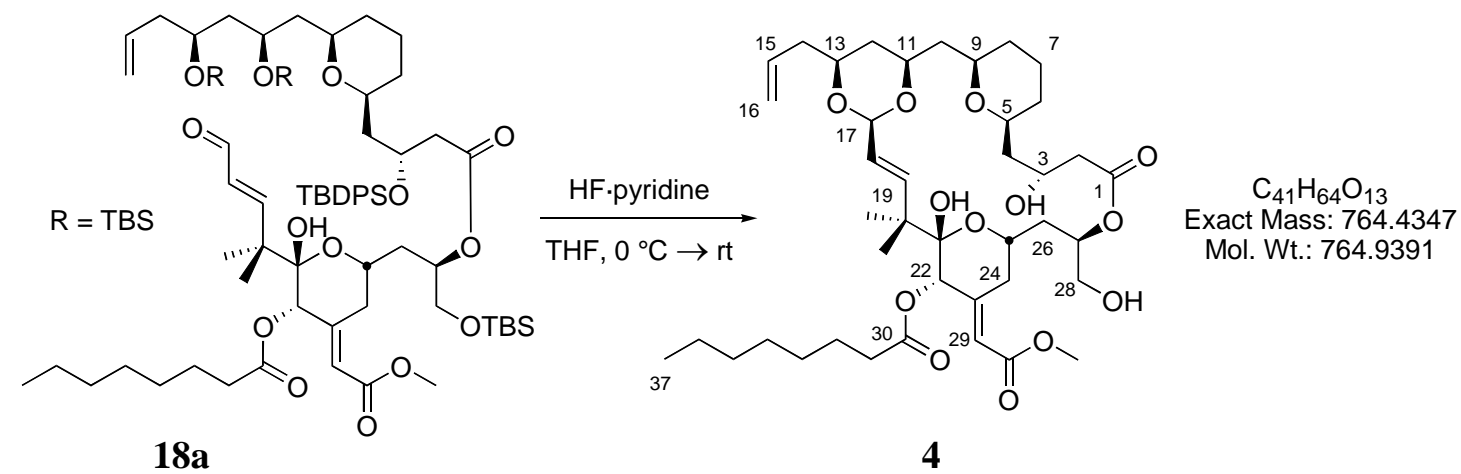

To a solution of seco aldehyde 18a $(5.7 \mathrm{mg}, 4.2 \mu \mathrm{mol})$ in THF $(1.1 \mathrm{~mL})$, under $\mathrm{N}_{2}$ at $0^{\circ} \mathrm{C}$ in a $10 \mathrm{~mL}$ polypropylene vial, was added HF.pyridine $(0.21 \mathrm{~mL})$ dropwise over $1 \mathrm{~min}$. The reaction was stirred for $5 \mathrm{~min}$ at $0{ }^{\circ} \mathrm{C}$, then the bath was removed and the reaction was allowed to stir for $27 \mathrm{~h}$ at room temperature. The reaction solution was transferred to a larger $50 \mathrm{~mL}$ propylene vial using EtOAc $(10 \mathrm{~mL})$. Water $(5 \mathrm{~mL})$ was added followed by slow addition of sat aq. $\mathrm{NaHCO}_{3}$ until gas evolution ceased ( $7 \mathrm{~mL}$, vigorous bubbling!). The layers were separated and the aqueous phase was extracted with EtOAc $(3 \times 5 \mathrm{~mL})$. The combined organic layers were dried over anhydrous $\mathrm{Na}_{2} \mathrm{SO}_{4}$, filtered and concentrated in vacuo. Chromatography on silica $(60 \rightarrow 80 \%$ EtOAc / pentane) gave $3.1 \mathrm{mg}$ of the completed analog 4 as a white amorphous solid (97\%).

Data for 4:

$\mathbf{R}_{f}=0.59$ (70\% EtOAc / petroleum ether) - one black spot w/ p-anisaldehyde stain.

IR (thin film): $3453,3313,2933,2857,1722,1665,1463,1435,1407,1382,1362,1345,1298$, $1257,1230,1156,1108,1088,1054,1002,983,919,889,862,755 \mathrm{~cm}^{-1}$.

${ }^{1} \mathbf{H}$ NMR $\left(600 \mathrm{MHz}, \mathrm{CDCl}_{3}\right): \delta 5.98(1 \mathrm{H}, \mathrm{d}, J=1.9 \mathrm{~Hz}, \mathrm{C} 19), 5.96(1 \mathrm{H}, \mathrm{d}, J=16.0 \mathrm{~Hz}, \mathrm{C} 19)$, $5.78(1 \mathrm{H}$, dddd, $J=6.5,7.9,10.3,16.9 \mathrm{~Hz}, \mathrm{C} 15), 5.40(1 \mathrm{H}, \mathrm{dd}, J=7.6,16.0 \mathrm{~Hz}, \mathrm{C} 18), 5.35(1 \mathrm{H}$, ddt, $J=5.7,12.3,2.8 \mathrm{~Hz}, \mathrm{C} 27), 5.14(1 \mathrm{H}, \mathrm{s}, \mathrm{C} 22), 5.12(1 \mathrm{H}, \mathrm{s}, \mathrm{C} 21-\mathrm{OH}), 5.06(2 \mathrm{H}, \mathrm{m}, \mathrm{C} 16)$, $5.02(1 \mathrm{H}, \mathrm{d}, J=7.5 \mathrm{~Hz}, \mathrm{C} 17), 4.50(1 \mathrm{H}, \mathrm{d}, 12.0 \mathrm{~Hz}, \mathrm{C} 3-\mathrm{OH}), 4.18(1 \mathrm{H}, \mathrm{m}, \mathrm{C} 3), 4.05(1 \mathrm{H}, \mathrm{tt}, J=$ 2.6, 11.3 Hz, C25), $3.85(2 \mathrm{H}, \mathrm{m}, \mathrm{C} 11 \& \mathrm{C} 28), 3.75(1 \mathrm{H}, \mathrm{m}, \mathrm{C} 13), 3.69(1 \mathrm{H}, \mathrm{dd}, J=2.3,13.8 \mathrm{~Hz}$, C24), $3.67\left(3 \mathrm{H}, \mathrm{s},-\mathrm{OCH}_{3}\right), 3.65(1 \mathrm{H}, \mathrm{m}, \mathrm{C} 28), 3.52(1 \mathrm{H}, \mathrm{m}, \mathrm{C} 5), 3.42(1 \mathrm{H}, \mathrm{m}, \mathrm{C} 9), 2.51(2 \mathrm{H}, \mathrm{m}$, C2), $2.41(1 \mathrm{H}, \mathrm{m}, \mathrm{C} 14), 2.29(2 \mathrm{H}, \mathrm{m}, \mathrm{C} 31), 2.18(1 \mathrm{H}, \mathrm{dt}, J=14.8,7.5 \mathrm{~Hz}, \mathrm{C} 14), 2.01(4 \mathrm{H}, \mathrm{m}$, $\mathrm{C} 4, \mathrm{C} 24, \mathrm{C} 26$ \& $\mathrm{C} 28-\mathrm{OH}), 1.80$ (3H, m, C7, C10 \& $\mathrm{C} 26), 1.42-1.64$ (8H, m, C4, C6, C7, C8, $\mathrm{C} 10, \mathrm{C} 12$ \& C32), $1.33(1 \mathrm{H}, \mathrm{m}, \mathrm{C} 12), 1.26(10 \mathrm{H}, \mathrm{m}, \mathrm{C} 6, \mathrm{C} 8$ \& $\mathrm{C} 33-\mathrm{C} 36), 1.17$ (3H, s, -CH $\mathbf{H}_{3}$, $1.03\left(3 \mathrm{H}, \mathrm{s},-\mathrm{CH}_{3}\right), 0.87(3 \mathrm{H}, \mathrm{t}, J=6.8 \mathrm{~Hz}, \mathrm{C} 37)$.

${ }^{13} \mathrm{C}$ NMR $\left(100 \mathrm{MHz}, \mathrm{CDCl}_{3}\right): \delta 172.7,172.1,167.0,151.7,142.4,133.8,125.5,119.8,117.4$, 102.5, 98.9, 78.7, 75.8, 75.7, 75.4, 74.1, 71.7, 68.5, 65.8, 64.4, 51.1, 45.2, 42.9, 42.5, 40.4, 39.9, 37.5, 35.9, 34.6, 31.6, 31.4, 31.3, 31.0, 29.0, 28.9, 24.7, 24.3, 23.0, 22.6, 19.2, 14.1.

HRMS (ESI+): Calculated for $\mathrm{C}_{41} \mathrm{H}_{64} \mathrm{O}_{13} \mathrm{Na}[\mathrm{M}+\mathrm{Na}]$ : 787.4245. Found: 787.4235.

$[\alpha]_{D}^{23}=-13.3^{\circ}\left(c=0.31, \mathrm{CHCl}_{3}\right)$. 

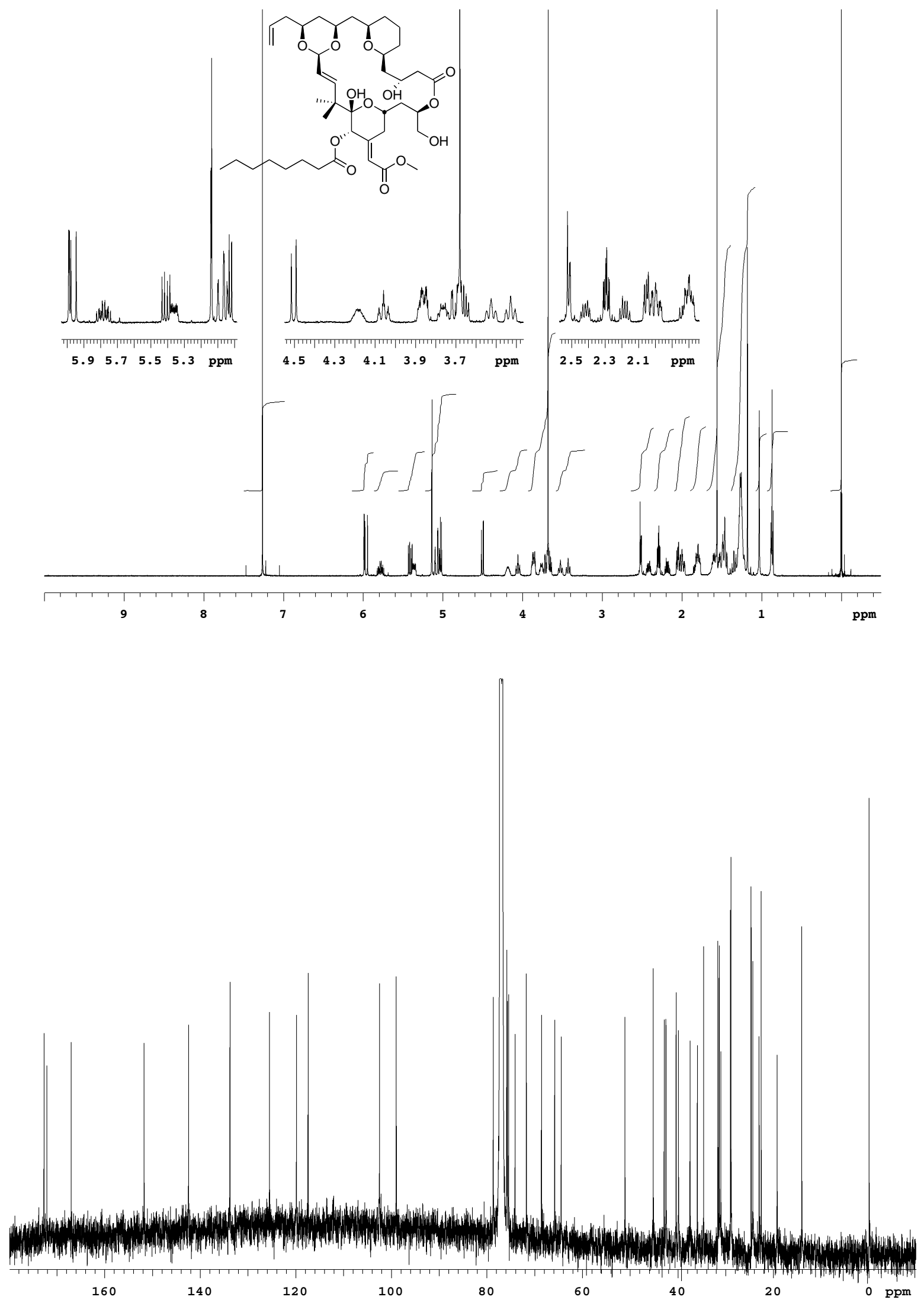
PKC-Isozyme Mixture Binding Assay. In a conical vial, a buffer solution was made consisting of Tris- $\mathrm{HCl}(1 \mathrm{M}, 1 \mathrm{~mL}, \mathrm{pH} 7.4), \mathrm{KCl}(1 \mathrm{M}, 2 \mathrm{~mL}), \mathrm{CaCl}_{2}(0.1 \mathrm{M}, 30 \mu \mathrm{L})$, and bovine serum albumin $\left(40 \mathrm{mg}\right.$ ), which was then diluted to $20 \mathrm{~mL}$ with $\mathrm{H}_{2} \mathrm{O}$ and stored on ice. Phosphatidyl serine vesicles $\left(140 \mu \mathrm{L}, 25 \mathrm{mg} / \mathrm{mL}\right.$ in $\left.\mathrm{CHCl}_{3}\right)$ were prepared by removing the chloroform under a stream of nitrogen. The prepared buffer solution was added $(3.5 \mathrm{~mL})$ and the solution was sonicated (Branson Sonifier 250, power $=6,40 \%$ duty cycle) four times for $30 \mathrm{~s}$ with a $30 \mathrm{~s}$ rest in between sonications and stored on ice as a cloudy solution. A mixture of PKC isozymes, prepared by the method of Mochly-Rosen, ${ }^{\mathrm{iii}}$ was prepared by adding a solution of PKC isozymes $(600 \mu \mathrm{L})$ to the prepared buffer solution $(14 \mathrm{~mL})$ and stored on ice. Final assay vials were prepared in triplicate by adding the phosphatidyl serine solution $(60 \mu \mathrm{L})$, PKC solution $(200 \mu \mathrm{L})$, and diluted test compound $(20 \mu \mathrm{L})$. Non-specific binding was measured by the substitution of analog $1(75 \mathrm{mM}, 20 \mu \mathrm{L})$ for the test compound. A set of vials was also prepared with the absence of any analog as a control. Lastly, ${ }^{3} \mathrm{H}-\mathrm{PDBU}(30 \mathrm{nM}, 20 \mu \mathrm{L})$ was added to the vials, the solutions vortexed, incubated at $37{ }^{\circ} \mathrm{C}$ for $90 \mathrm{~min}$, and then put on ice for $15 \mathrm{~min}$. Filters (Whatman GF/B $21 \mathrm{~mm}$ ) were prepared by soaking in a solution of aq. polyethyleneamine $(10 \%$ by volume, $6 \mathrm{~mL})$ and water $(200 \mathrm{~mL})$ for $90 \mathrm{~min}$. Prepared buffer solution $(0.5 \mathrm{~mL}, 20 \mathrm{mM}$ Tris) was added to the vials and then the solutions were individually filtered through the previously prepared filters. The filters were washed slowly with buffer $(4.5 \mathrm{~mL})$, placed into scintillation vials, and filled with Universol scintillation fluid $(5 \mathrm{~mL})$. The filters were immediately counted in a scintillation counter (Beckman LS 6000SC). Counts per minute were averaged among three trials at each concentration. The data was then plotted using Prism by GraphPad Software and an $\mathrm{IC}_{50}$ (the concentration of analog required to displace half of the specific $\mathrm{PDBu}$ binding to $\mathrm{PKC}$ ) was calculated. The $\mathrm{IC}_{50}$ then allowed determination of the $K_{\mathrm{i}}$ for the analog from the equation: $K_{\mathrm{i}}=\mathrm{IC}_{50} /(1+[\mathrm{PDBu}]) / K_{\mathrm{d}}$ of $\left.\mathrm{PDBu}\right)$. The $K_{\mathrm{d}}$ of $\left[{ }^{3} \mathrm{H}\right]-\mathrm{PDBu}$ was determined under identical conditions to be $1.17 \mathrm{nM}$.

\footnotetext{
${ }^{i}$ Wender, P. A.; Mayweg, A. V. W.; VanDeusen, C. L. Org. Lett. 2003, 5, 277-279.

${ }^{i i}$ Rychnovsky, S. D.; Rogers, B. N.; Richardson, T. I. Acc. Chem. Res. 1998, 31, 9-17.

iii Mochly-Rosen, D.; Koshland, Jr., D. E. J. Biol. Chem. 1987, 262, 2291-2297.
} 